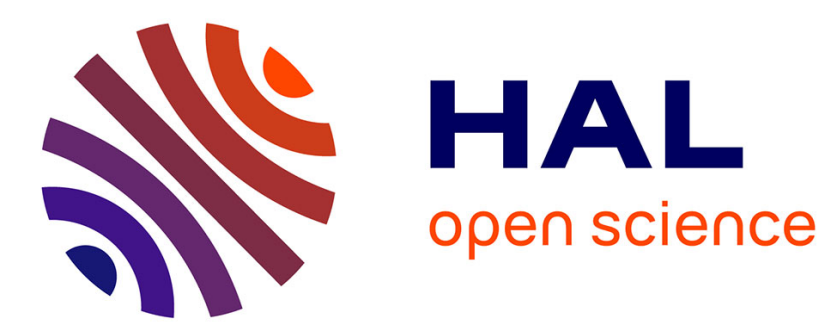

\title{
First-Kind Boundary Integral Equations for the Hodge-Helmholtz Operator
}

\author{
Xavier Claeys, Ralf Hiptmair
}

\section{To cite this version:}

Xavier Claeys, Ralf Hiptmair. First-Kind Boundary Integral Equations for the Hodge-Helmholtz

Operator. SIAM Journal on Mathematical Analysis, 2019. hal-02425385

\section{HAL Id: hal-02425385 \\ https://hal.science/hal-02425385}

Submitted on 30 Dec 2019

HAL is a multi-disciplinary open access archive for the deposit and dissemination of scientific research documents, whether they are published or not. The documents may come from teaching and research institutions in France or abroad, or from public or private research centers.
L'archive ouverte pluridisciplinaire HAL, est destinée au dépôt et à la diffusion de documents scientifiques de niveau recherche, publiés ou non, émanant des établissements d'enseignement et de recherche français ou étrangers, des laboratoires publics ou privés. 


\title{
FIRST-KIND BOUNDARY INTEGRAL EQUATIONS FOR THE HODGE-HELMHOLTZ OPERATOR
}

\author{
X. CLAEYS* AND R. HIPTMAIR ${ }^{\dagger}$
}

\begin{abstract}
We adapt the variational approach to the analysis of first-kind boundary integral equations associated with strongly elliptic partial differential operators from [M. COSTABEL, Boundary integral operators on Lipschitz domains: Elementary results, SIAM J. Math. Anal., 19 (1988), pp. 613-626.] to the (scaled) Hodge-Helmholtz equation curl curl $\mathbf{u}-\eta \nabla \operatorname{div} \mathbf{u}-\kappa^{2} \mathbf{u}=0$, $\eta>0, \operatorname{Im} \kappa^{2} \geq 0$, on Lipschitz domains in 3D Euclidean space, supplemented with natural complementary boundary conditions, which, however, fail to bring about strong ellipticity.

Nevertheless, a boundary integral representation formula can be found, from which we can derive boundary integral operators. They induce bounded and coercive sesqui-linear forms in the natural energy trace spaces for the Hodge-Helmholtz equation. We can establish precise conditions on $\eta, \kappa$ that guarantee unique solvability of the two first-kind boundary integral equations associated with the natural boundary value problems for the Hodge-Helmholtz equations. Particular attention will be given to the case $\kappa=0$.
\end{abstract}

Key words. Maxwell's Equations; static limit, Hodge-Laplacian; potential representations, jump relations, first-kind boundary integral equations; coercive integral equations.

AMS subject classifications. 31A10, 45A05, 45E05.

1. Introduction. Relying on a vector potential $\mathbf{A}$ and a scalar potential $\Phi$ and employing Lorentz gauge, the linear Maxwell's equations in frequency domain driven by a source current $\mathbf{j}$ with angular frequency $\omega>0$ can be recast as [12, Sect. 4]

$$
\begin{aligned}
& \operatorname{curl} \boldsymbol{\mu}(\boldsymbol{x})^{-1} \operatorname{curl} \mathbf{A}+i \omega \boldsymbol{\epsilon}(\boldsymbol{x}) \nabla \Phi-\omega^{2} \boldsymbol{\epsilon}(\boldsymbol{x}) \mathbf{A}=\mathbf{j}, \\
& \operatorname{div}(\boldsymbol{\epsilon}(\boldsymbol{x}) \mathbf{A})+i \omega \Phi=0
\end{aligned}
$$

Straightforward elimination of $\Phi$ yields the vector second-order partial differential equation (PDE) $\operatorname{curl} \boldsymbol{\mu}(\boldsymbol{x})^{-1} \operatorname{curl} \mathbf{A}-\boldsymbol{\epsilon}(\boldsymbol{x}) \nabla \operatorname{div}(\boldsymbol{\epsilon}(\boldsymbol{x}) \mathbf{A})-\omega^{2} \boldsymbol{\epsilon} \mathbf{A}=\mathbf{j}$. Thus, in the case of homogeneous and isotropic materials, where both $\boldsymbol{\mu}$ and $\boldsymbol{\epsilon}$ are constant multiples of the identity matrix, A will satisfy the second-order Hodge-Helmholtz equation

$$
\operatorname{curl} \operatorname{curl} \mathbf{A}-\eta \nabla \operatorname{div} \mathbf{A}-\kappa^{2} \mathbf{A}=0
$$

for some $\kappa, \eta>0$. Its principal part is the negative Hodge-Laplacian $-\Delta_{\eta}:=\operatorname{curl}$ curl $-\eta \nabla$ div.

This work is dedicated to the derivation and analysis of first-kind boundary integral equations (BIEs) related to boundary value problems for (1.2). We aim to adapt the modern variational treatment of BIEs for strongly elliptic boundary value problems, as pioneered in [27, 14], and pursued, for instance, in the monograph [23], to the Hodge-Helmholtz equation. More precisely, we investigate boundary value problems and BIEs in a Hilbert space framework supplied by suitable Sobolev (energy) spaces and associated trace spaces. This permits us to work on domains with merely Lipschitz boundaries. Special attention will also be paid to the static limit $\kappa=0$.

Novelty. A fairly mature theory of (exterior) boundary value problems for $\Delta_{\eta}$ has been developed, see, e.g., [37, Section 4]. Vast literature is also devoted to the Hodge-Laplacian $\Delta:=\delta \mathrm{d}+\mathrm{d} \delta$ acting on differential forms on smooth manifolds. This

*Sorbonne Université, Université Paris-Diderot SPC, CNRS, Inria, Laboratoire Jacques-Louis Lions, équipe Alpines, F-75005 Paris

†Seminar of Applied Mathematics, ETH Zürich 
approach is reviewed comprehensively in the books [24, 25] and [33]. In the former two volumes boundary integral equations play a central role, but the focus is on second-kind boundary integral equations set in $L^{p}$-type spaces on domain boundaries. This perspective requires analysis and techniques fundamentally different from what we present in this manuscript, which studies first-kind boundary integral equations. Apparently, those have received little attention so far.

To keep the presentation simple and accessible we confine ourselves to classical vector analysis on Euclidean space $\mathbb{R}^{3}$ and forgo developments in an exterior calculus setting on manifolds. This is certainly feasible based on results for Maxwell's equations $[21,36]$.

Outline. Our main results, stated in Corollaries 6.9 and 6.12 , will be a comprehensive understanding of the mapping properties in Sobolev-Hilbert trace spaces of first-kind boundary integral operators connected with "natural" elliptic boundary value problems (BVPs) for the Hodge-Helmholtz equation. These BVPs along with the relevant trace operators are elucidated in Section 3. The associated boundary representation formula is given in Equation (4.6), based on potentials whose properties are examined in Section 5. Then we are equipped to tackle the coercivity of variational first-kind boundary integral operators in Section 6. In the last section we identify kernels of boundary integrals and link them to topological properties of boundaries for the pure Hodge-Laplacian $(\kappa=0)$.

\section{Preliminaries.}

2.1. Lipschitz domains. In the sequel, $\Omega \subset \mathbb{R}^{3}$ will refer to a Lipschitz domain such that either $\Omega$ or $\mathbb{R}^{3} \backslash \bar{\Omega}$ is bounded. Recall from [23, Ch. 3] that, by definition, a Lipschitz domain $\Omega$ is an open set such that for any $\boldsymbol{x} \in \partial \Omega$ there exists a neighbourhood $\boldsymbol{x} \in U \subset \mathbb{R}^{3}$ and a Lipschitz function $\psi: \mathbb{R}^{2} \rightarrow \mathbb{R}$ such that, in a certain cartesian coordinate system $\boldsymbol{z}=\left(z_{1}, z_{2}, z_{3}\right)$ with origin at $\boldsymbol{x}$, we have $U \cap \partial \Omega=\left\{\boldsymbol{z}=\left(z_{1}, z_{2}, z_{3}\right) \epsilon\right.$ $\left.U \mid z_{3}=\psi\left(z_{1}, z_{2}\right)\right\}$, and $U \cap \Omega=\left\{\boldsymbol{z}=\left(z_{1}, z_{2}, z_{3}\right) \in U \mid z_{3}<\psi\left(z_{1}, z_{2}\right)\right\}$. The boundary will be denoted $\Gamma:=\partial \Omega$. According to Rademacher's theorem (see [16, Thm. 3.1.6]) it admits an essentially bounded unit normal vector field $\boldsymbol{n} \in \mathrm{L}^{\infty}(\Gamma)$, directed toward the exterior of $\Omega$.

2.2. Domain based function spaces. The subsequent analysis will make repeated use of various function spaces, so we dedicate the present section to recalling, in detail, some of those spaces that are now classical in the literature dealing with electromagnetics, e.g., [1], [26, Ch. 3], [18, Sect. 2.4]. We first introduce volume based function spaces. As usual, $\mathrm{L}^{2}(\Omega)$ refers to square integrable (either scalar of vector valued) fields over $\Omega$. For any integer $k \geq 0$, we also consider Sobolev spaces

$$
\begin{aligned}
& \mathrm{H}^{k}(\Omega):=\left\{v \in \mathrm{L}^{2}(\Omega) \mid \partial_{\boldsymbol{x}}^{\alpha} v \in \mathrm{L}^{2}(\Omega) \forall \alpha \in \mathbb{N}^{2} \text { with }|\alpha| \leq k\right\}, \\
& \|u\|_{\mathrm{H}^{k}(\Omega)}^{2}=\sum_{|\alpha| \leq k}\left\|\partial_{\boldsymbol{x}}^{\alpha} u\right\|_{\mathrm{L}^{2}(\Omega)}^{2} .
\end{aligned}
$$

The space $H_{0}^{1}(\Omega)$ will refer to the closure of $\mathscr{C}_{0}^{\infty}(\Omega):=\left\{\left.\varphi\right|_{\Omega} \in \mathscr{C}^{\infty}\left(\mathbb{R}^{3}\right), \operatorname{supp}(\varphi) \subset\right.$ $\Omega\}$ with respect to the norm \|\|$_{\mathrm{H}^{1}(\Omega)}$. We will consider the following functional spaces that are rather classical in the analysis of Maxwell's equations (see [18, 26] for example)

$$
\begin{aligned}
& \mathbf{H}(\operatorname{div}, \Omega):=\left\{\mathbf{u} \in \mathrm{L}^{2}(\Omega)^{3} \mid \operatorname{div}(\mathbf{u}) \in \mathrm{L}^{2}(\Omega)\right\} \\
& \mathbf{H}(\operatorname{curl}, \Omega):=\left\{\mathbf{u} \in \mathrm{L}^{2}(\Omega)^{3} \mid \operatorname{curl}(\mathbf{u}) \in \mathrm{L}^{2}(\Omega)^{3}\right\}
\end{aligned}
$$

These spaces will be equipped with the norm given by $\|\mathbf{u}\|_{\mathfrak{d}, \Omega}^{2}=\|\mathbf{u}\|_{\mathrm{L}^{2}(\Omega)}^{2}+\|\mathfrak{d}(\mathbf{u})\|_{\mathrm{L}^{2}(\Omega)}^{2}$ where $\mathfrak{d}=\operatorname{div}$ or curl. The space $\mathbf{H}_{0}(\mathbf{c u r l}, \Omega)\left(\operatorname{resp} . \mathbf{H}_{0}(\operatorname{div}, \Omega)\right)$ will refer to the 
closure of $\mathscr{C}_{0}^{\infty}(\Omega)^{3}$ with respect to the norm \|\|$_{\operatorname{curl}, \Omega}\left(\right.$ resp. \|\|$\left._{\operatorname{div}, \Omega}\right)$. We will also consider the following spaces

$$
\begin{aligned}
& \mathbf{X}(\Omega):=\mathbf{H}(\operatorname{curl}, \Omega) \cap \mathbf{H}(\operatorname{div}, \Omega), \\
& \text { with }\|\mathbf{v}\|_{\mathbf{X}(\Omega)}^{2}:=\|\mathbf{v}\|_{\operatorname{curl}, \Omega}^{2}+\|\mathbf{v}\|_{\operatorname{div}, \Omega}^{2} .
\end{aligned}
$$

We will also use the space $\mathbf{H}\left(\operatorname{curl}^{2}, \Omega\right):=\{\mathbf{u} \in \mathbf{H}(\operatorname{curl}, \Omega) \mid \operatorname{curl}(\mathbf{u}) \in \mathbf{H}(\operatorname{curl}, \Omega)\}$ equipped with $\|\mathbf{u}\|_{\operatorname{curl}^{2}, \Omega}^{2}:=\|\mathbf{u}\|_{\operatorname{curl}, \Omega}^{2}+\|\operatorname{curl}(\mathbf{u})\|_{\operatorname{curl}, \Omega}^{2}$ as well as the $\operatorname{space} \mathbf{H}(\nabla \operatorname{div}, \Omega):=$ $\left\{\mathbf{u} \in \mathbf{H}(\operatorname{div}, \Omega) \mid \operatorname{div}(\mathbf{u}) \in \mathrm{H}^{1}(\Omega)\right\}$ equipped with $\|\mathbf{u}\|_{\nabla \operatorname{div}, \Omega}^{2}:=\|\mathbf{u}\|_{\operatorname{div}, \Omega}^{2}+\|\operatorname{div}(\mathbf{u})\|_{\mathrm{H}^{1}(\Omega)}^{2}$. We will also need the space $\mathrm{H}^{1}(\Delta, \Omega):=\left\{u \in \mathrm{H}^{1}(\Omega) \mid \nabla u \in \mathrm{H}(\operatorname{div}, \Omega)\right\}$ endowed with the norm $\|u\|_{\Delta, \Omega}^{2}:=\|u\|_{\mathrm{H}^{1}(\Omega)}^{2}+\|\Delta u\|_{\mathrm{L}^{2}(\Omega)}^{2}$. Finally, if $\mathrm{H}$ refers to any of the previously mentionned spaces, $\mathrm{H}_{\text {loc }}$ will consist in all functions/fields $v$ such that $\varphi v \in \mathrm{H}$ for any $\varphi \in \mathscr{C}^{\infty}\left(\mathbb{R}^{3}\right)$ such that $\operatorname{supp}(\varphi)$ is bounded.

When studying Maxwell's equations in domains with boundaries only admitting Lipschitz regularity, it is essential to keep in mind that $\mathrm{H}^{1}(\Omega)^{3} \neq \mathbf{X}(\Omega)$, although $\mathrm{H}_{0}^{1}(\Omega)^{3}=\mathbf{H}_{0}(\mathbf{c u r l}, \Omega) \cap \mathbf{H}_{0}(\operatorname{div}, \Omega)$, see [1]. This does not prevent us from applying Fredholm theory to variational formulations of Maxwell's equations, as

$$
\begin{aligned}
& \mathbf{X}_{\mathrm{N}}(\Omega):=\mathbf{H}_{0}(\operatorname{curl}, \Omega) \cap \mathbf{H}(\operatorname{div}, \Omega), \\
& \mathbf{X}_{\mathrm{T}}(\Omega):=\mathbf{H}(\mathbf{c u r l}, \Omega) \cap \mathbf{H}_{0}(\operatorname{div}, \Omega),
\end{aligned}
$$

are both compactly embedded in $\mathrm{L}^{2}(\Omega)^{3}$ for bounded domains $\Omega$, see $[29,35,30,2]$.

2.3. Scalar trace spaces. Derivation and analysis of boundary integral equation heavily relies on trace-type operators. Therefore we now discuss these operators, the associated function spaces, and appropriate lifting maps. We consider traces for both scalar and vector valued fields. In the sequel, we denote the boundary of the domain $\Omega$ by $\Gamma:=\partial \Omega$. We first introduce the so-called (scalar) Dirichlet trace operator $\tau_{\mathrm{D}}$ defined by

$$
\tau_{\mathrm{D}}(\varphi):=\left.\varphi\right|_{\Gamma} \quad \forall \varphi \in \mathscr{C}^{\infty}(\bar{\Omega})
$$

This operator gives rise to a continuous linear operator mapping $\mathrm{H}^{1}(\Omega)$ into $\mathrm{L}^{2}(\Gamma)$, see [32, Thm. 2.6.8]. The kernel of $\tau_{\mathrm{D}}$ is exactly $\mathrm{H}_{0}^{1}(\Omega)$. The range of this map, denoted $\mathrm{H}^{1 / 2}(\Gamma)$, is a Hilbert space when equipped with the norm

$$
\|p\|_{\mathrm{H}^{1 / 2}(\Gamma)}:=\inf \left\{\|v\|_{\mathrm{H}^{1}(\Omega)}\left|v \in \mathrm{H}^{1}(\Omega), v\right|_{\Gamma}=p\right\} .
$$

Occasionally, we will also consider $\tau_{\mathrm{D}}$ as mapping $\mathrm{H}^{1}(\Omega)^{3}$ to $\mathrm{H}^{1 / 2}(\Gamma)^{3}$ i.e. $\tau_{\mathrm{D}}(\mathbf{u}):=$ $\left(\tau_{\mathrm{D}}\left(u_{1}\right), \tau_{\mathrm{D}}\left(u_{2}\right), \tau_{\mathrm{D}}\left(u_{3}\right)\right)$ where $\mathbf{u}=\left(u_{1}, u_{2}, u_{3}\right) \in \mathrm{H}^{1}(\Omega)^{3}$. We will also use the unique continuous trace operator $\gamma_{\mathrm{D}}: \mathbf{H}(\nabla \operatorname{div}, \Omega) \rightarrow \mathrm{H}^{1 / 2}(\Gamma)$ defined, for all $\mathbf{p} \in \mathscr{C}^{\infty}(\bar{\Omega})^{3}$, by the formula

$$
\gamma_{\mathrm{D}}(\mathbf{p}):=\left.\operatorname{div}(\mathbf{p})\right|_{\Gamma} .
$$

The dual space of $\mathrm{H}^{1 / 2}(\Gamma)$ will be denoted $\mathrm{H}^{-1 / 2}(\Gamma)$, and it is naturally equipped with the canonical dual norm $p \mapsto \sup _{v \in \mathrm{H}^{1 / 2}(\Gamma) \backslash\{0\}}|\langle v, p\rangle| /\|v\|_{\mathrm{H}^{1 / 2}(\Gamma)}$. In connection with this trace space, let us recall that the normal component trace operator

$$
\gamma_{\mathrm{N}}(\mathbf{v}):=\left.\boldsymbol{n} \cdot \mathbf{v}\right|_{\Gamma}
$$

induces a continuous linear surjective map from $\mathbf{H}(\operatorname{div}, \Omega)$ onto $\mathrm{H}^{-1 / 2}(\Gamma)$. Its kernel is exactly $\mathbf{H}_{0}(\operatorname{div}, \Omega)$. As a consequence, the norm on $\mathrm{H}^{-1 / 2}(\Gamma)$ may alternatively be given by the following expression

$$
\|q\|_{\mathrm{H}^{-1 / 2}(\Gamma)}:=\inf \left\{\|\mathbf{p}\|_{\operatorname{div}, \Omega}|\mathbf{p} \in \mathbf{H}(\operatorname{div}, \Omega), \boldsymbol{n} \cdot \mathbf{p}|_{\Gamma}=q\right\} .
$$


Finallty, we will also need the usual scalar Neumann trace $\tau_{\mathrm{N}}: \mathrm{H}_{\text {loc }}^{1}(\Delta, \bar{\Omega}) \rightarrow \mathrm{H}^{-1 / 2}(\Gamma)$ as the unique operator satisfying

$$
\tau_{\mathrm{N}}(\varphi):=\left.\boldsymbol{n} \cdot \nabla \varphi\right|_{\Gamma} \quad \forall \varphi \in \mathscr{C}^{\infty}(\bar{\Omega}) .
$$

2.4. Vector tangential trace spaces. Now we wish to briefly recall the precise definition of the trace space associated to $\mathbf{H}(\mathbf{c u r l}, \Omega)$. Following $[8,6,5]$, we first consider the intermediate space $\mathbf{H}_{\mathrm{T}}^{1 / 2}(\Gamma):=\left\{\gamma_{\mathrm{T}}(\mathbf{u}):=\boldsymbol{n} \times\left(\tau_{\mathrm{D}}(\mathbf{u}) \times \boldsymbol{n}\right), \mathbf{u} \in \mathrm{H}^{1}(\Omega)^{3}\right\}$ that is a Banach space when equipped with the following associated quotient norm

$$
\begin{aligned}
& \|\mathbf{v}\|_{\mathbf{H}_{\mathrm{T}}^{1 / 2}(\Gamma)}:=\inf \left\{\|\mathbf{u}\|_{\mathrm{H}^{1}(\Omega)} \mid \gamma_{\mathrm{T}}(\mathbf{u})=\mathbf{v}\right\} \\
& \text { where } \quad \gamma_{\mathrm{T}}(\mathbf{v}):=\boldsymbol{n} \times\left(\left.\mathbf{v}\right|_{\Gamma} \times \boldsymbol{n}\right)
\end{aligned}
$$

As was thoroughly described in Section 2 of [8], the space $\mathbf{H}_{\mathrm{T}}^{1 / 2}(\Gamma)$ is not left invariant under the action of the operator $\mathbf{v} \mapsto \boldsymbol{n} \times \mathbf{v}$. This leads us to introduce the space of "rotated traces"

$$
\mathbf{H}_{\mathrm{R}}^{1 / 2}(\Gamma):=\left\{\mathbf{v} \mid \boldsymbol{n} \times \mathbf{v} \in \mathbf{H}_{\mathrm{T}}^{1 / 2}(\Gamma)\right\}, \quad \text { with norm }\|\mathbf{v}\|_{\mathbf{H}_{\mathrm{R}}^{1 / 2}(\Gamma)}:=\|\boldsymbol{n} \times \mathbf{v}\|_{\mathbf{H}_{\mathrm{T}}^{1 / 2}(\Gamma)} .
$$

Then we define the associated topological dual spaces and associated canonical norms adopting the following notational convention (in accordance with $[8,9]$ ),

$$
\mathbf{H}_{\mathrm{T}}^{-1 / 2}(\Gamma):=\mathbf{H}_{\mathrm{R}}^{1 / 2}(\Gamma)^{\prime} \quad, \quad \mathbf{H}_{\mathrm{R}}^{-1 / 2}(\Gamma):=\mathbf{H}_{\mathrm{T}}^{1 / 2}(\Gamma)^{\prime} .
$$

The operator $\mathbf{v} \mapsto \boldsymbol{n} \times \mathbf{v}$ isometrically maps $\mathbf{H}_{\mathrm{T}}^{-1 / 2}(\Gamma)$ onto $\mathbf{H}_{\mathrm{R}}^{-1 / 2}(\Gamma)$. In the case where the functional ${ }^{*} v \mapsto\langle\mathbf{p}, \boldsymbol{n} \times \nabla v\rangle, v \in \mathscr{C}^{\infty}(\bar{\Omega})$ is continuous with respect to \|\|$_{\mathrm{H}^{1}(\Omega)}$, we denote by $\operatorname{curl}_{\Gamma}(\mathbf{p})$ the only element of $\mathrm{H}^{-1 / 2}(\Gamma)$ satisfying $\left\langle\operatorname{curl}_{\Gamma}(\mathbf{p}), \tau_{\mathrm{D}}(v)\right\rangle:=$ $-\langle\mathbf{p}, \boldsymbol{n} \times \nabla v\rangle, \forall v \in \mathscr{C}^{\infty}(\bar{\Omega})$. We will then consider the following trace space

$$
\begin{aligned}
& \mathbf{H}^{-1 / 2}(\operatorname{curl}, \Gamma):=\left\{\mathbf{p} \in \mathbf{H}_{\mathrm{T}}^{-1 / 2}(\Gamma) \mid \operatorname{curl}_{\Gamma}(\mathbf{p}) \in \mathrm{H}^{-1 / 2}(\Gamma)\right\}, \\
& \text { with norm }\|\mathbf{v}\|_{\mathbf{H}^{-1 / 2}(\operatorname{curl}, \Gamma)}:=\|\mathbf{v}\|_{\mathbf{H}_{\mathrm{T}}^{-1 / 2}(\Gamma)}+\left\|\operatorname{curl}_{\Gamma}(\mathbf{v})\right\|_{\mathrm{H}^{-1 / 2}(\Gamma)} .
\end{aligned}
$$

It was also established in $\left[8\right.$, Thm. 4.1] that the trace operator $\gamma_{\mathrm{T}}(\mathbf{v}):=\boldsymbol{n} \times\left(\left.\mathbf{v}\right|_{\Gamma} \times \boldsymbol{n}\right)$ defined in (2.5) induces a continuous linear and surjective operator mapping $\mathbf{H}(\mathbf{c u r l}, \Omega)$ onto $\mathbf{H}^{-1 / 2}(\operatorname{curl}, \Gamma)$. Moreover the kernel of this trace operator is exactly $\mathbf{H}_{0}(\mathbf{c u r l}, \Omega)$. As a consequence the norm in (2.8) may be replaced by

$$
\|\mathbf{p}\|_{\mathbf{H}^{-1 / 2}(\operatorname{curl}, \Gamma)}:=\inf \left\{\|\mathbf{v}\|_{\mathbf{c u r l}, \Omega} \mid \mathbf{v} \in \mathbf{H}(\operatorname{curl}, \Omega), \gamma_{\mathrm{T}}(\mathbf{v})=\mathbf{p}\right\} .
$$

Define the surface gradient by $\nabla_{\Gamma} \tau_{\mathrm{D}}(v):=\gamma_{\mathrm{T}}(\nabla v)$ for any $v \in \mathrm{H}^{1}(\Gamma)$. This operator continuously maps $\mathrm{H}^{1 / 2}(\Gamma)$ into $\mathbf{H}^{-1 / 2}(\operatorname{curl}, \Gamma)$, see $[8$, Prop. 3.6]. Similarly, curl $\Gamma$ continuously maps $\mathbf{H}^{-1 / 2}(\operatorname{curl}, \Gamma)$ into $\mathrm{H}^{-1 / 2}(\Gamma)$. We can also set $\operatorname{div}_{\Gamma}(\mathbf{u}):=\operatorname{curl}_{\Gamma}(\boldsymbol{n} \times$ $\mathbf{u})$, and introduce the rotated version of $(2.8)$

$$
\begin{aligned}
& \mathbf{H}^{-1 / 2}(\operatorname{div}, \Gamma):=\left\{\mathbf{v} \in \mathbf{H}_{\mathrm{R}}^{-1 / 2}(\Gamma) \mid \operatorname{div}_{\Gamma}(\mathbf{v}) \in \mathrm{H}^{-1 / 2}(\Gamma)\right\} \\
& \text { with } \quad\|\mathbf{v}\|_{\mathbf{H}^{-1 / 2}(\operatorname{div}, \Gamma)}:=\|\mathbf{v}\|_{\mathbf{H}_{\mathrm{R}}^{-1 / 2}(\Gamma)}+\left\|\operatorname{div}_{\Gamma}(\mathbf{v})\right\|_{\mathrm{H}^{-1 / 2}(\Gamma)} .
\end{aligned}
$$

\footnotetext{
${ }^{*}$ Throughout brackets $\langle\cdot, \cdot\rangle$ designate bilinear duality pairings with respect to an $\mathrm{L}^{2}$-type pivot
} space. 
We have $\mathbf{v} \in \mathbf{H}^{-1 / 2}(\operatorname{div}, \Gamma)$ if and only if $\boldsymbol{n} \times \mathbf{v} \in \mathbf{H}^{-1 / 2}(\operatorname{curl}, \Gamma)$. Besides, $\mathbf{H}^{-1 / 2}(\operatorname{div}, \Gamma)$ can be identified with the topological dual to $\mathbf{H}^{-1 / 2}(\operatorname{curl}, \Gamma)$ with duality pairing $\mathbf{u}, \mathbf{v} \mapsto\langle\mathbf{u}, \mathbf{v}\rangle:=\int_{\Gamma} \mathbf{u} \cdot \mathbf{v} d \sigma$. With the previous definitions we have the surface Green formula: for any $\mathbf{u} \in \mathbf{H}^{-1 / 2}(\operatorname{div}, \Gamma)$, and any $v \in \mathrm{H}^{1 / 2}(\Gamma)$,

$$
\left\langle\mathbf{u}, \nabla_{\Gamma} v\right\rangle=-\left\langle\operatorname{div}_{\Gamma}(\mathbf{u}), v\right\rangle .
$$

Note that the trace $\mathbf{u} \mapsto \boldsymbol{n} \times\left.\mathbf{u}\right|_{\Gamma}$ induces a continuous surjective operator mapping $\mathbf{H}(\operatorname{curl}, \Omega)$ onto $\mathbf{H}^{-1 / 2}(\operatorname{div}, \Gamma)$. We will also need the continuous trace operator $\gamma_{\mathrm{R}}$ : $\mathbf{H}\left(\mathbf{c u r l}^{2}, \Omega\right) \rightarrow \mathbf{H}^{-1 / 2}(\operatorname{div}, \Gamma)$ given by the formula

$$
\gamma_{\mathrm{R}}(\mathbf{v}):=\boldsymbol{n} \times\left.\operatorname{curl}(\mathbf{v})\right|_{\Gamma} .
$$

2.5. Lifting maps. In this paragraph we wish to introduce lifting maps, aka, continuous right inverses, for each one of the four trace operators introduced with (2.2), (2.3), (2.5) and (2.11).

Dirichlet lifting.. For any $v \in \mathrm{H}^{1 / 2}(\Gamma)$, define $\varrho_{\mathrm{D}}(v)$ as the unique element of $\mathbf{H}(\operatorname{div}, \Omega)$ satisfying $\int_{\Omega} \operatorname{div}\left(\varrho_{\mathrm{D}}(v)\right) \operatorname{div}(\mathbf{p})+\varrho_{\mathrm{D}}(v) \mathbf{p} d \boldsymbol{x}=\left\langle\gamma_{\mathrm{N}}(\mathbf{p}), v\right\rangle$ for all $\mathbf{p} \in \mathbf{H}(\operatorname{div}, \Omega)$. Lax-Milgram's lemma shows that $\varrho_{\mathrm{D}}(v)$ is well-defined for any $v \in \mathrm{H}^{1 / 2}(\Gamma)$ and continuously depends on $v$. Moreover routine verifications based on Green's formula show that it satisfies the equations

$$
\begin{array}{ll}
\nabla \operatorname{div} \varrho_{\mathrm{D}}(v)-\varrho_{\mathrm{D}}(v)=0 & \text { in } \Omega \\
\operatorname{curl}\left(\varrho_{\mathrm{D}}(v)\right)=0 & \text { in } \Omega \\
\gamma_{\mathrm{D}}\left(\varrho_{\mathrm{D}}(v)\right)=v & \text { on } \Gamma .
\end{array}
$$

Neumann lifting.. For any given $q \in \mathrm{H}^{-1 / 2}(\Gamma)$ let $\varrho_{\mathrm{N}}(q)$ refer to the unique element of $\mathbf{H}(\operatorname{div}, \Omega)$ achieving the minimum in Definition (2.4) i.e. $\left\|\varrho_{\mathrm{N}}(q)\right\|_{\operatorname{div}, \Omega}=$ $\|q\|_{\mathrm{H}^{-1 / 2}(\Gamma)}$. Then Euler's equation associated to this minimization problem reads $\int_{\Omega} \operatorname{div}\left(\varrho_{\mathrm{N}}(q)\right) \operatorname{div}(\mathbf{p})+\varrho_{\mathrm{N}}(q) \mathbf{p} d \boldsymbol{x}=0$ for all $\mathbf{p} \in \mathrm{H}_{0}(\operatorname{div}, \Omega)$. From this we conclude that $\varrho_{\mathrm{N}}: \mathrm{H}^{-1 / 2}(\Gamma) \rightarrow \mathrm{H}(\operatorname{div}, \Omega)$ is a continuous map satisfying

$$
\begin{array}{ll}
\nabla \operatorname{div} \varrho_{\mathrm{N}}(q)-\varrho_{\mathrm{N}}(q)=0 & \text { in } \Omega \\
\operatorname{curl}\left(\varrho_{\mathrm{N}}(q)\right)=0 & \text { in } \Omega \\
\gamma_{\mathrm{N}}\left(\varrho_{\mathrm{N}}(q)\right)=q & \text { on } \Gamma .
\end{array}
$$

Tangential lifting.. For any given $\mathbf{p} \in \mathbf{H}^{-1 / 2}(\operatorname{curl}, \Gamma)$ let $\varrho_{\mathrm{T}}(\mathbf{p})$ refer to the unique element of $\mathbf{H}(\mathbf{c u r l}, \Omega)$ achieving the minimum in Definition (2.9) i.e. $\left\|\varrho_{\mathrm{T}}(\mathbf{p})\right\|_{\mathbf{c u r l}, \Omega}=$ $\|\mathbf{p}\|_{\mathbf{H}^{-1 / 2}(\operatorname{curl}, \Gamma)}$. Then Euler's equation associated to this minimization problem reads $\int_{\Omega} \operatorname{curl} \varrho_{\mathrm{T}}(\mathbf{p}) \cdot \operatorname{curl}(\mathbf{v})+\varrho_{\mathrm{T}}(\mathbf{p}) \mathbf{v} d \boldsymbol{x}=0$ for all $\mathbf{v} \in \mathbf{H}_{0}(\mathbf{c u r l}, \Omega)$. From this we conclude that $\varrho_{\mathrm{T}}: \mathbf{H}^{-1 / 2}(\operatorname{curl}, \Gamma) \rightarrow \mathbf{H}(\operatorname{curl}, \Omega)$ is a continuous map satisfying

$$
\begin{array}{ll}
\operatorname{curl}^{2} \varrho_{\mathrm{T}}(\mathbf{p})+\varrho_{\mathrm{T}}(\mathbf{p})=0 & \text { in } \Omega \\
\operatorname{div}\left(\varrho_{\mathrm{T}}(\mathbf{p})\right)=0 & \text { on } \Omega \\
\gamma_{\mathrm{T}}\left(\varrho_{\mathrm{T}}(\mathbf{p})\right)=\mathbf{p} & \text { on } \Gamma .
\end{array}
$$

Rotated tangential lifting.. For any $\mathbf{p} \in \mathbf{H}^{-1 / 2}(\operatorname{div}, \Gamma)$, define $\varrho_{\mathrm{R}}(\mathbf{p})$ as the unique element of $\mathbf{H}(\mathbf{c u r l}, \Omega)$ satisfying $\int_{\Omega} \operatorname{curl} \varrho_{\mathrm{R}}(\mathbf{p}) \cdot \operatorname{curl}(\mathbf{v})+\varrho_{\mathrm{R}}(\mathbf{p}) \mathbf{v} d \boldsymbol{x}=-\left\langle\mathbf{p}, \gamma_{\mathrm{T}}(\mathbf{v})\right\rangle$ for all $\mathbf{v} \in \mathbf{H}(\mathbf{c u r l}, \Omega)$. Lax-Milgram lemma shows that $\varrho_{\mathrm{R}}(\mathbf{p})$ is well defined for any 
$\mathbf{p} \in \mathbf{H}^{-1 / 2}(\operatorname{div}, \Gamma)$ and continuously depends on $\mathbf{p}$. In addition, Green's formula shows that it satisfies

$$
\begin{array}{ll}
\operatorname{curl}^{2} \varrho_{\mathrm{R}}(\mathbf{p})+\varrho_{\mathrm{R}}(\mathbf{p})=0 & \text { in } \Omega \\
\operatorname{div}\left(\varrho_{\mathrm{R}}(\mathbf{p})\right)=0 & \text { on } \Omega \\
\gamma_{\mathrm{R}}\left(\varrho_{\mathrm{R}}(\mathbf{p})\right)=\mathbf{p} & \text { on } \Gamma .
\end{array}
$$

2.6. Mean value and jump trace operators. The operators $\tau_{*}, *=\mathrm{D}, \mathrm{N}$ and $\gamma_{*}, *=\mathrm{D}, \mathrm{N}, \mathrm{R}, \mathrm{T}$, correspond to traces of functions taken on $\Gamma=\partial \Omega$ from the interior of the domain $\Omega$. We will also consider operators $\tau_{*, c}, *=\mathrm{D}, \mathrm{N}$ and $\gamma_{*, c}, *=\mathrm{D}, \mathrm{N}, \mathrm{R}, \mathrm{T}$ defined in the same manner, except that the trace is taken from the interior of $\mathbb{R}^{3} \backslash \bar{\Omega}$. In the definition of these exterior trace operators, the normal field $\boldsymbol{n}$ remains the same, pointing toward the exterior of $\Omega$. We will also consider jump and mean value operators defined by

$$
\begin{aligned}
& {\left[\gamma_{*}(\mathbf{v})\right]:=\gamma_{*}(\mathbf{v})-\gamma_{*, c}(\mathbf{v})} \\
& \left\{\gamma_{*}(\mathbf{v})\right\}:=\frac{1}{2}\left(\gamma_{*}(\mathbf{v})+\gamma_{*, c}(\mathbf{v})\right), \quad *=\mathrm{D}, \mathrm{N}, \mathrm{R}, \mathrm{T} .
\end{aligned}
$$

We define $\left[\tau_{*}(v)\right]$ and $\left\{\tau_{*}(v)\right\}, *=\mathrm{D}, \mathrm{N}$ accordingly.

3. Boundary value problems for the Hodge-Helmholtz operator. The principal part of the Hodge-Helmholtz partial differential operator is the negative Hodge Laplacian

$$
\begin{aligned}
& -\boldsymbol{\Delta}_{\eta} \mathbf{u}:=\operatorname{curl}(\operatorname{curl} \mathbf{u})-\eta \nabla(\operatorname{div} \mathbf{u}) \\
& \mathbf{u} \in \mathbf{X}(\Delta, \Omega)=\operatorname{Dom}\left(\boldsymbol{\Delta}_{\eta}\right):=\mathbf{H}\left(\operatorname{curl}^{2}, \Omega\right) \cap \mathbf{H}(\nabla \operatorname{div}, \Omega) .
\end{aligned}
$$

It will determine appropriate boundary conditions for the $\mathrm{PDE}-\boldsymbol{\Delta}_{\eta} \mathbf{u}-\kappa^{2} \mathbf{u}=0$ on $\partial \Omega$. From now, for the remainder of this manuscript we fix the parameter $\eta$ to some strictly positive value.

The Hodge-Helmholtz operator is a symmetric, closed, densely defined operator on $\mathrm{L}^{2}(\Omega)^{3}$. Its domain $\mathbf{X}(\boldsymbol{\Delta}, \Omega)$ will be equipped with the norm $\|\mathbf{u}\|_{\mathbf{X}(\boldsymbol{\Delta}, \Omega)}^{2}:=\|\mathbf{u}\|_{\mathbf{c u r l}^{2}, \Omega^{+}}^{2}$ $\|\mathbf{u}\|_{\nabla \operatorname{div}, \Omega}^{2}$, which turns it into a Hilbert space.

3.1. Green's formula and boundary conditions. We embark on a variational treatment of the operator (3.1), which is based on Green's formula associated with it: using the compact notations for the trace operators from $(2.2),(2.3),(2.5)$, and (2.11) we find

$$
\begin{aligned}
\int_{\Omega} \mathbf{u} \cdot\left(-\boldsymbol{\Delta}_{\eta} \mathbf{v}\right) d \boldsymbol{x} & =\int_{\Omega} \mathbf{u} \cdot(\operatorname{curl}(\operatorname{curl} \mathbf{v})-\eta \nabla(\operatorname{div} \mathbf{v})) d \boldsymbol{x} \\
& =a_{\eta}(\mathbf{u}, \mathbf{v})-\eta\left\langle\gamma_{\mathrm{N}} \mathbf{u}, \gamma_{\mathrm{D}} \mathbf{v}\right\rangle+\left\langle\gamma_{\mathrm{R}} \mathbf{v}, \gamma_{\mathrm{T}} \mathbf{u}\right\rangle \\
& =\int_{\Omega}\left(-\boldsymbol{\Delta}_{\eta} \mathbf{u}\right) \cdot \mathbf{v} d \boldsymbol{x}-\eta\left\langle\gamma_{\mathrm{N}} \mathbf{u}, \gamma_{\mathrm{D}} \mathbf{v}\right\rangle+\eta\left\langle\gamma_{\mathrm{D}} \mathbf{u}, \gamma_{\mathrm{N}} \mathbf{v}\right\rangle \\
& +\left\langle\gamma_{\mathrm{R}} \mathbf{v}, \gamma_{\mathrm{T}} \mathbf{u}\right\rangle-\left\langle\gamma_{\mathrm{R}} \mathbf{u}, \gamma_{\mathrm{T}} \mathbf{v}\right\rangle
\end{aligned}
$$

for $\mathbf{u}, \mathbf{v} \in \mathbf{X}(\boldsymbol{\Delta}, \Omega)$ and with the positive semi-definite symmetric bilinear form

$$
a_{\eta}(\mathbf{u}, \mathbf{v}):=\int_{\Omega} \operatorname{curl} \mathbf{u} \cdot \operatorname{curl} \mathbf{v}+\eta \operatorname{div} \mathbf{u} \operatorname{div} \mathbf{v} d \boldsymbol{x} .
$$

A theory of boundary integral equations associated with the second-order operator $-\boldsymbol{\Delta}_{\eta}-\kappa^{2}$ Id will entail a pair of "dual" (in the sense of $\mathrm{L}^{2}(\Gamma)$-duality of their image spaces) trace operators, roles played for the scalar Laplacian by the well-known 
Dirichlet and Neumann (conormal) traces. However, as is evident from (3.2), all four trace operators $\gamma_{\mathrm{T}}, \gamma_{\mathrm{N}}, \gamma_{\mathrm{R}}$, and $\gamma_{\mathrm{D}}$ matter for $-\boldsymbol{\Delta}_{\eta}$. The criterion for a meaningful selection of two pairs of complementary boundary conditions is

1. that they are linked to variational problems for $(\mathbf{u}, \mathbf{v}) \mapsto a_{\eta}(\mathbf{u}, \mathbf{v})+\int_{\Omega} \mathbf{u} \cdot \mathbf{v} d \boldsymbol{x}$ that spawn a compact solution operator in $\mathrm{L}^{2}(\Omega)^{3}$, and

2 . that they allow imposing arbitrary functions from the trace spaces as inhomogeneous boundary conditions.

The first option is the partitioning $\left\{\gamma_{\mathrm{T}}, \gamma_{\mathrm{N}}, \gamma_{\mathrm{R}}, \gamma_{\mathrm{D}}\right\}=\left\{\gamma_{\mathrm{T}}, \gamma_{\mathrm{N}}\right\} \cup\left\{\gamma_{\mathrm{R}}, \gamma_{\mathrm{D}}\right\}$. From Section 2.3 we learn that the corresponding trace spaces $\mathbf{H}^{-1 / 2}(\operatorname{curl}, \Gamma) \times \mathrm{H}^{-1 / 2}(\Gamma)$ and $\mathbf{H}^{-1 / 2}(\operatorname{div}, \Gamma) \times \mathrm{H}^{1 / 2}(\Gamma)$ are $\mathrm{L}^{2}(\Gamma)$-duals of each other. These sets of traces induce the boundary value problems $\left(\mathbf{f} \in \mathrm{L}^{2}(\Omega)^{3}\right)$

$$
\begin{gathered}
-\boldsymbol{\Delta}_{\eta} \mathbf{u}+\mathbf{u}=\mathbf{f} \quad \text { in } \Omega, \\
\gamma_{\mathrm{T}}(\mathbf{u})=0, \gamma_{\mathrm{N}}(\mathbf{u})=0 \text { on } \Gamma,
\end{gathered} \quad \text { and } \quad \begin{array}{r}
-\boldsymbol{\Delta}_{\eta} \mathbf{u}+\mathbf{u}=\mathbf{f} \quad \text { in } \Omega, \\
\gamma_{\mathrm{R}}(\mathbf{u})=0, \gamma_{\mathrm{D}}(\mathbf{u})=0 \text { on } \Gamma,
\end{array}
$$

respectively. The associated variational problems are posed on the spaces $\mathrm{H}_{0}^{1}(\Omega)^{3}=$ $\mathbf{H}_{0}(\operatorname{curl}, \Omega) \cap \mathbf{H}_{0}(\operatorname{div}, \Omega)$ and $\mathbf{X}(\Omega)$. As $\mathbf{X}(\Omega)$ fails to be compactly embedded in $\mathrm{L}^{2}(\Omega)^{3}$ even for bounded $\Omega$, the right BVP in (3.4) will not give rise to a compact mapping $\mathrm{L}^{2}(\Omega)^{3} \rightarrow \mathrm{L}^{2}(\Omega)^{3}, \mathbf{f} \mapsto \mathbf{u}$.

Remark 3.1. We point out that for the left BVP from (3.4) general inhomogeneous boundary values in $\mathbf{H}^{-1 / 2}(\operatorname{curl}, \Gamma) \times \mathrm{H}^{-1 / 2}(\Gamma)$ cannot be imposed. If $\Gamma$ is smooth, then setting $\gamma_{\mathrm{T}} \mathbf{u}=0$ will imply $\mathbf{u} \in \mathbf{X}_{\mathrm{N}}(\Omega) \subset \mathrm{H}^{1}(\Omega)^{3}$ [1, Cor. 2.15], which, in turns, means $\gamma_{\mathrm{N}}(\mathbf{u}) \in \mathrm{H}^{1 / 2}(\Gamma): \gamma_{\mathrm{N}} \mathbf{u}$ cannot be made to match general boundary values $\in \mathrm{H}^{-1 / 2}(\Omega)$.

The second option is the partitioning $\left\{\gamma_{\mathrm{T}}, \gamma_{\mathrm{N}}, \gamma_{\mathrm{R}}, \gamma_{\mathrm{D}}\right\}=\left\{\gamma_{\mathrm{T}}, \gamma_{\mathrm{D}}\right\} \cup\left\{\gamma_{\mathrm{R}}, \gamma_{\mathrm{N}}\right\}$, which also accommodates $\mathrm{L}^{2}(\Gamma)$-duality of trace spaces on both sides. This grouping of traces spawns the two boundary value problems $\left(\mathbf{f} \in \mathrm{L}^{2}(\Omega)^{3}\right)$

$$
\begin{gathered}
-\boldsymbol{\Delta}_{\eta} \mathbf{u}+\mathbf{u}=\mathbf{f} \quad \text { in } \Omega, \\
\gamma_{\mathrm{T}}(\mathbf{u})=0, \gamma_{\mathrm{D}}(\mathbf{u})=0 \text { on } \Gamma,
\end{gathered} \text { and } \quad \begin{gathered}
-\boldsymbol{\Delta}_{\eta} \mathbf{u}+\mathbf{u}=\mathbf{f} \quad \text { in } \Omega, \\
\gamma_{\mathrm{R}}(\mathbf{u})=0, \gamma_{\mathrm{N}}(\mathbf{u})=0 \text { on } \Gamma,
\end{gathered}
$$

respectively. The underlying variational problems are posed on the space $\mathbf{X}_{\mathrm{N}}(\Omega)$ and $\mathbf{X}_{\mathrm{T}}(\Omega)$, which are both compactly embedded in $\mathrm{L}^{2}(\Omega)^{3}$ for bounded $\Omega$, see $\S 2.2$. Hence, both solution operators $\mathbf{f} \mapsto \mathbf{u}$ will be compact in $\mathrm{L}^{2}(\Omega)^{3}$ on bounded domains. We conclude that this grouping of trace operators provides the right pair of "Dirichlet" and "Neumann" traces for the Hodge-Laplacian. For the sake of brevity, we write for the associated "Dirichlet" and "Neumann" trace spaces

$$
\mathcal{H}_{\mathrm{D}}(\Gamma):=\mathbf{H}^{-\frac{1}{2}}(\operatorname{curl}, \Gamma) \times \mathrm{H}^{+\frac{1}{2}}(\Gamma) \quad, \quad \mathcal{H}_{\mathrm{N}}(\Gamma):=\mathbf{H}^{-\frac{1}{2}}(\operatorname{div}, \Gamma) \times \mathrm{H}^{-\frac{1}{2}}(\Gamma) .
$$

Remember that $\mathrm{H}^{-1 / 2}(\Gamma)$ is by definition the $\mathrm{L}^{2}(\Gamma)$-dual to $\mathrm{H}^{+1 / 2}(\Gamma)$ and (see [8] for example) $\mathbf{H}^{-1 / 2}(\operatorname{div}, \Gamma)$ is $\mathrm{L}^{2}(\Gamma)$-dual to $\mathbf{H}^{-1 / 2}(\operatorname{curl}, \Gamma)$. As a consequence $\mathcal{H}_{\mathrm{N}}(\Gamma)$ is topologically dual to $\mathcal{H}_{\mathrm{D}}(\Gamma)$ with respect to the canonical pairing induced by the combined $\mathrm{L}^{2}(\Gamma)$ inner products

$$
\langle(\mathbf{u}, p),(\mathbf{v}, q)\rangle:=\int_{\Gamma} \mathbf{u} \cdot \mathbf{v}+p q d \sigma, \quad \forall(\mathbf{u}, p) \in \mathcal{H}_{\mathrm{D}}(\Gamma), \quad \forall(\mathbf{v}, q) \in \mathcal{H}_{\mathrm{N}}(\Gamma)
$$

As concise notation we introduce two continuous vector trace operators $\mathcal{T}_{\mathrm{D}}: \mathbf{X}(\boldsymbol{\Delta}, \Omega) \rightarrow$ $\mathcal{H}_{\mathrm{D}}(\Gamma)$ and $\mathcal{T}_{\mathrm{N}}: \mathbf{X}(\boldsymbol{\Delta}, \Omega) \rightarrow \mathcal{H}_{\mathrm{N}}(\Gamma)$. As explained above, they should be regarded as generalized counterparts of Dirichlet/Neumann traces, and are given by the formulas

$$
\mathcal{T}_{\mathrm{D}}(\mathbf{u}):=\left(\gamma_{\mathrm{T}}(\mathbf{u}), \eta \gamma_{\mathrm{D}}(\mathbf{u})\right) \quad, \quad \mathcal{T}_{\mathrm{N}}(\mathbf{u}):=\left(\gamma_{\mathrm{R}}(\mathbf{u}), \gamma_{\mathrm{N}}(\mathbf{u})\right)
$$


They permit us to rewrite (3.2) in the compact form

$\int_{\Omega} \mathbf{u} \cdot \boldsymbol{\Delta}_{\eta}(\mathbf{v})-\mathbf{v} \cdot \boldsymbol{\Delta}_{\eta}(\mathbf{u}) d \boldsymbol{x}=\left\langle\left\langle\mathcal{T}_{\mathrm{N}}(\mathbf{u}), \mathcal{T}_{\mathrm{D}}(\mathbf{v})\right\rangle-\left\langle\left\langle\mathcal{T}_{\mathrm{N}}(\mathbf{v}), \mathcal{T}_{\mathrm{D}}(\mathbf{u})\right\rangle\right\rangle \quad \forall \mathbf{u}, \mathbf{v} \in \mathbf{X}(\boldsymbol{\Delta}, \Omega)\right.$

The current selection of boundary conditions also makes it possible to prescribe arbitrary "Dirichlet" or "Neumann" data as confirmed by the following finding.

Lemma 3.2. There exists a continuous lifting map $\mathcal{R}_{\mathrm{D}}: \mathcal{H}_{\mathrm{D}}(\Gamma) \rightarrow \mathbf{X}(\boldsymbol{\Delta}, \Omega)$ (resp. $\mathcal{R}_{\mathrm{N}}: \mathcal{H}_{\mathrm{N}}(\Gamma) \rightarrow \mathbf{X}(\boldsymbol{\Delta}, \Omega)$ ) such that $\mathcal{T}_{\mathrm{D}} \cdot \mathcal{R}_{\mathrm{D}}=\mathrm{Id}$ (resp. $\left.\mathcal{T}_{\mathrm{N}} \cdot \mathcal{R}_{\mathrm{N}}=\mathrm{Id}\right)$. In particular $\mathcal{T}_{\mathrm{D}}$ and $\mathcal{T}_{\mathrm{N}}$ are surjective.

Proof:

Observe that the range of the liftings $\varrho_{*}, *=\mathrm{D}, \mathrm{N}, \mathrm{T}, \mathrm{R}$ is contained in $\mathbf{X}(\boldsymbol{\Delta}, \Omega)$ according to (2.13), (2.12), (2.14) and (2.15). As can be checked straightforwardly, for any $(\boldsymbol{\alpha}, q) \in \mathcal{H}_{\mathrm{D}}(\Gamma)$, the operator $\mathcal{R}_{\mathrm{D}}(\boldsymbol{\alpha}, q):=\varrho_{\mathrm{T}}(\boldsymbol{\alpha})+\eta^{-1}\left(\mathrm{Id}-\varrho_{\mathrm{T}} \cdot \gamma_{\mathrm{T}}\right) \cdot \varrho_{\mathrm{D}}(q)$ fullfils the assertions of the lemma. Similarly, for any $(\boldsymbol{\beta}, p) \in \mathcal{H}_{\mathrm{N}}(\Gamma)$, the operator $\mathcal{R}_{\mathrm{N}}(\boldsymbol{\beta}, p):=\varrho_{\mathrm{N}}(p)+\left(\mathrm{Id}-\varrho_{\mathrm{N}} \cdot \gamma_{\mathrm{N}}\right) \cdot \varrho_{\mathrm{R}}(\boldsymbol{\beta})$ meets the requirements of the lemma.

Like in the case of more standard trace operators, we will need to consider operators $\mathcal{T}_{\mathrm{D}, c}=\left(\gamma_{\mathrm{T}, c}, \eta \gamma_{\mathrm{D}, c}\right)$ and $\mathcal{T}_{\mathrm{N}, c}=\left(\gamma_{\mathrm{R}, c}, \gamma_{\mathrm{N}, c}\right)$ defined in the same manner as $\mathcal{T}_{\mathrm{D}}, \mathcal{T}_{\mathrm{N}}$ except that the traces are taken from $\mathbb{R}^{3} \backslash \bar{\Omega}$. We will also need mean value and jump operators defined by

$$
\left[\mathcal{T}_{*}\right]=\mathcal{T}_{*}-\mathcal{T}_{*, c} \quad \text { and } \quad\left\{\mathcal{T}_{*}\right\}=\frac{1}{2}\left(\mathcal{T}_{*}+\mathcal{T}_{*, c}\right) . \quad *=\mathrm{D}, \mathrm{N}
$$

These operators will naturally come into play when considering transmission problems and boundary integral operators associated with the Hodge-Helmholtz equation.

Remark 3.3. The significance of the boundary conditions in (3.5) has long been recognized. They are prominently covered in [24, Sect. 1.1] and [25, Ch. 5]. From [33, Sect. 1.6] we learn that they render (3.5) elliptic in the sense of Lopatinskii-Sapiro. That $\mathcal{T}_{\mathrm{D}}$ and $\mathcal{T}_{\mathrm{N}}$ are "natural" for the Hodge-Laplacian is also highlighted in [17, §2.2.2] and $[15, \S 1 . c]$. In the context of exterior calculus $\mathcal{T}_{\mathrm{D}}$ and $\mathcal{T}_{\mathrm{N}}$ are converted into each other by applying the Hodge operator.

Remark 3.4. As key difference to the variational theory of boundary value problems for strongly elliptic partial differential operators [23, Ch. 4] is the failure of a crucial identity:

$$
\left\langle\left\langle\mathcal{T}_{\mathrm{N}}(\mathbf{u}), \mathcal{T}_{\mathrm{D}}(\mathbf{u})\right\rangle \neq a_{\eta}(\mathbf{u}, \mathbf{u}) \quad \text { for some } \mathbf{u} \in \mathbf{X}(\boldsymbol{\Delta}, \Omega), \boldsymbol{\Delta}_{\eta} \mathbf{u}=0\right.
$$

Changing a sign in Definition (3.7) of the duality pairing could fix this, but would thwart (3.8).

3.2. Spectrum of $\boldsymbol{\Delta}_{\eta}$ in bounded domains. For the Hodge-Helmholtz operator $-\boldsymbol{\Delta}_{\eta}-\kappa^{2}$ Id equipped with either set of boundary conditions from (3.5), uniqueness of solutions will break down for $\kappa^{2}$ contained in the spectrum of $\boldsymbol{\Delta}_{\eta}$ (plus boundary conditions), which is a pure point spectrum for bounded $\Omega$. Therefore we study the spectrum of the Hodge-Laplace operator in the bounded domain $\Omega$ with either Dirichlet or Neumann boundary conditions,

$$
\begin{aligned}
\mathfrak{S}\left(\boldsymbol{\Delta}_{\eta}^{*}, \Omega\right):=\{\lambda \in \mathbb{C} \mid \exists \mathbf{v} \in \mathbf{X}(\Omega) \backslash\{0\}, \\
\left.-\boldsymbol{\Delta}_{\eta} \mathbf{v}=\lambda \mathbf{v} \text { in } \Omega, \mathcal{T}_{*}(\mathbf{v})=0 \text { on } \partial \Omega\right\}, \quad \text { where } *=\mathrm{D}, \mathrm{N} .
\end{aligned}
$$


Due to the compact embedding of $\mathbf{X}_{\mathrm{T}}(\Omega)$ and $\mathbf{X}_{\mathrm{N}}(\Omega)$ into $\mathrm{L}^{2}(\Omega)^{3}$, cf. [30], the operator $\boldsymbol{\Delta}_{\eta}^{*}$ has a self-adjoint compact resolvent and, consequently, $\mathfrak{S}\left(\boldsymbol{\Delta}_{\eta}^{*}, \Omega\right)$ is a discrete subset of $\mathbb{R}_{+}$accumulating only at $\infty$.

Proposition 3.5. Assume that $\Omega$ is bounded. Then $\mathfrak{S}\left(\boldsymbol{\Delta}_{\eta}^{\mathrm{D}}, \Omega\right)=\Lambda_{\mathrm{T}} \cup \eta \Lambda_{\mathrm{D}}$ where

$$
\begin{aligned}
& \Lambda_{\mathrm{D}}:=\left\{\lambda \in \mathbb{C} \mid \exists v \in \mathrm{H}^{1}(\Omega) \backslash\{0\},-\Delta v=\lambda v \text { in } \Omega, \tau_{\mathrm{D}}(v)=0 \text { on } \partial \Omega\right\}, \\
& \Lambda_{\mathrm{T}}:=\left\{\lambda \in \mathbb{C} \mid \exists \mathbf{v} \in \mathbf{H}(\mathbf{c u r l}, \Omega) \backslash\{0\}, \mathbf{c u r l}^{2}(\mathbf{v})=\lambda \mathbf{v} \text { in } \Omega, \gamma_{T}(v)=0 \text { on } \partial \Omega\right\} .
\end{aligned}
$$

\section{Proof:}

Pick some $\lambda \in \mathfrak{S}\left(\boldsymbol{\Delta}_{\eta}^{\mathrm{D}}, \Omega\right)$ and consider $\mathbf{v} \in \mathbf{X}(\Omega) \backslash\{0\}$ such that $-\boldsymbol{\Delta}_{\eta}^{\mathrm{D}} \mathbf{v}=\lambda \mathbf{v}$ in $\Omega$ and $\mathcal{T}_{\mathrm{D}}(\mathbf{v})=0$. If $\operatorname{div}(\mathbf{v})=0$ in $\Omega$, since in particular $\gamma_{\mathrm{T}}(v)=0$ on $\partial \Omega$, we conclude that $\lambda \in \Lambda_{\mathrm{T}}$. Otherwise, we have $\lambda \operatorname{div}(\mathbf{v})=-\operatorname{div}\left(\boldsymbol{\Delta}_{\eta} \mathbf{v}\right)=-\eta \Delta \operatorname{div}(\mathbf{v})$ in $\Omega$, and then $\lambda \in \eta \Lambda_{\mathrm{D}}$. This shows that $\mathfrak{S}\left(\boldsymbol{\Delta}_{\eta}^{\mathrm{D}}, \Omega\right) \subset \Lambda_{\mathrm{T}} \cup \eta \Lambda_{\mathrm{D}}$.

Conversely, take $\lambda \in \Lambda_{\mathrm{T}}$, and consider $\mathbf{v} \in \mathbf{H}(\mathbf{c u r l}, \Omega) \backslash\{0\} \operatorname{satisfying}_{\mathbf{c u r l}^{2}}(\mathbf{v})=$ $\lambda \mathbf{v}$ and $\operatorname{div}(\mathbf{v})=0$ in $\Omega$, as well as $\gamma_{\mathrm{T}}(v)=0$ on $\partial \Omega$. We have in particular $\mathbf{v} \epsilon$ $\mathbf{X}(\Omega) \backslash\{0\}$, and $\lambda \mathbf{v}=\operatorname{curl}^{2}(\mathbf{v})=\operatorname{curl}^{2}(\mathbf{v})-\eta \nabla \operatorname{div}(\mathbf{v})=-\boldsymbol{\Delta}_{\eta} \mathbf{v}$ on $\Omega$. This proves $\Lambda_{\mathrm{T}} \subset \mathfrak{S}\left(\boldsymbol{\Delta}_{\eta}^{\mathrm{D}}, \Omega\right)$.

Finally take $\lambda \in \Lambda_{\mathrm{D}}$, and consider $v \in \mathrm{H}^{1}(\Omega) \backslash\{0\}$ such that $-\Delta v=\lambda v$ in $\Omega$, and $\tau_{\mathrm{D}}(v)=0$ on $\partial \Omega$. Set $\mathbf{w}:=\nabla v$. Clearly $\operatorname{curl}(\mathbf{w})=0$ and $\operatorname{div}(\mathbf{w})=-\lambda v \in \mathrm{L}^{2}(\Omega)$ so that $\mathbf{w} \in \mathbf{X}(\Omega)$. Moreover, if $\mathbf{w}=0$, then we must have $v=c$ for some $c \in \mathbb{C}$, and $c=0$ due to $\tau_{\mathrm{D}}(v)=0$ which is in contradiction with $v \neq 0$. So $\mathbf{w} \in \mathbf{X}(\Omega) \backslash\{0\}$. Clearly $\gamma_{\mathrm{T}}(\mathbf{w})=0$ on $\partial \Omega$ as $v$ admits a constant value (zero actually) on $\partial \Omega$, and $\operatorname{div}(\mathbf{w})=\Delta v=-\lambda v \in \mathrm{H}_{0}^{1}(\Omega)$ shows that $\tau_{\mathrm{D}}(\operatorname{div} \mathbf{w})=0$, so that $\mathcal{T}_{\mathrm{D}}(\mathbf{w})=0$. Finally, since curl $\mathbf{w}=0$, we have $-\boldsymbol{\Delta}_{\eta} \mathbf{w}=-\eta \nabla \operatorname{div}(\mathbf{w})=-\eta \nabla(\Delta v)=\eta \lambda \mathbf{w}$ in $\Omega$, so that $\eta \lambda \in \mathfrak{S}\left(\boldsymbol{\Delta}_{\eta}^{\mathrm{D}}, \Omega\right)$. This shows that $\eta \Lambda_{\mathrm{D}} \subset \mathfrak{S}\left(\boldsymbol{\Delta}_{\eta}^{\mathrm{D}}, \Omega\right)$ and concludes the proof.

An analogous result holds for Neumann type boundary conditions. We do not provide the proof, as it is very similar to the previous one.

Proposition 3.6. For bounded $\Omega$ we have $\mathfrak{S}\left(\Delta_{\eta}^{\mathrm{N}}, \Omega\right)=\Lambda_{\mathrm{R}} \cup \eta \Lambda_{\mathrm{N}}$, where

$$
\begin{aligned}
& \Lambda_{\mathrm{N}}:=\left\{\lambda \in \mathbb{C} \mid \exists v \in \mathrm{H}^{1}(\Omega) \backslash\{0\},\right.-\Delta v=\lambda v \text { in } \Omega, \tau_{\mathrm{N}}(v)=0 \text { on } \partial \Omega, \\
&\left.\int_{C} v d \boldsymbol{x}=0 \forall \text { connected components } C \text { of } \Omega .\right\}, \\
& \Lambda_{\mathrm{R}}:=\left\{\lambda \in \mathbb{C} \mid \exists \mathbf{v} \in \mathbf{H}(\mathbf{c u r l}, \Omega) \backslash\{0\}, \operatorname{curl}^{2}(\mathbf{v})=\lambda \mathbf{v} \text { in } \Omega, \gamma_{R}(v)=0 \text { on } \partial \Omega\right\} .
\end{aligned}
$$

Note that the sets $\Lambda_{\mathrm{D}}, \Lambda_{\mathrm{N}}$ do not contain 0 . On the other hand, if the second Betti number of $\Omega$ does not vanish, that is, if $\Omega$ has cavities, then $0 \in \Lambda_{\mathrm{T}}$. Conversely, $0 \in \Lambda_{\mathrm{R}}$, if the first Betti number of $\Omega$, which counts the number of handles, is non-zero.

Now that we have a precise description of the spectrum of the operator $\boldsymbol{\Delta}_{\eta}$, we can state well-posedness results for boundary value problems associated to this operator, as a direct application of a Fredholm alternative argument combined with the compact embedding of $\mathbf{X}_{\mathrm{T}}(\Omega)$ and $\mathbf{X}_{\mathrm{N}}(\Omega)$ into $\mathrm{L}^{2}(\Omega)^{3}$.

Proposition 3.7. Let $\Omega$ be a bounded Lipschitz domain. If $\kappa^{2} \in \mathbb{C} \backslash\left(\Lambda_{\mathrm{T}} \cup \eta \Lambda_{\mathrm{D}}\right)$, then for any $\mathbf{g} \in \mathcal{H}_{\mathrm{D}}(\Gamma)$ the following boundary value problem admits a unique solution,

$$
\left\{\begin{array}{l}
\mathbf{u} \in \mathbf{X}(\Omega)=\mathbf{H}(\operatorname{curl}, \Omega) \cap \mathbf{H}(\operatorname{div}, \Omega) \text { with } \\
-\boldsymbol{\Delta}_{\eta} \mathbf{u}-\kappa^{2} \mathbf{u}=0 \quad \text { in } \Omega \\
\mathcal{T}_{\mathrm{D}}(\mathbf{u})=\mathbf{g} \quad \text { on } \Gamma .
\end{array}\right.
$$


and the solution map is continuous: there exists a constant $C>0$ independent of

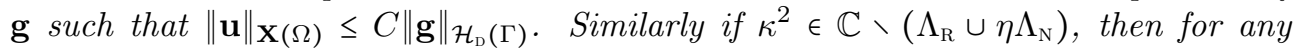
$\mathrm{g} \in \mathcal{H}_{\mathrm{N}}(\Gamma)$ the following problem admits a unique solution,

$$
\left\{\begin{array}{l}
\mathbf{u} \in \mathbf{X}(\Omega)=\mathbf{H}(\operatorname{curl}, \Omega) \cap \mathbf{H}(\operatorname{div}, \Omega) \text { with } \\
-\boldsymbol{\Delta}_{\eta} \mathbf{u}-\kappa^{2} \mathbf{u}=0 \quad \text { in } \Omega \\
\mathcal{T}_{\mathrm{N}}(\mathbf{u})=\mathbf{g} \quad \text { on } \Gamma .
\end{array}\right.
$$

Moreover the solution map is continuous: there exists a constant $C>0$ independent of $\mathbf{g}$ such that $\|\mathbf{u}\|_{\mathbf{X}(\Omega)} \leq C\|\mathbf{g}\|_{\mathcal{H}_{\mathrm{N}}(\Gamma)}$.

3.3. Conditions at infinity. Boundary value problems for $-\boldsymbol{\Delta}_{\eta}$ and $-\boldsymbol{\Delta}_{\eta}-\kappa^{2} \mathrm{Id}$ posed on unbounded domains $\Omega$ with compact Lipschitz boundary $\Gamma$, require suitable "boundary conditions at $\infty$ " to be well-posed.

3.3.1. (Static) case $\kappa=0$ : Hodge-Laplacian. To deal with static problems we consider the weighted space $\mathbf{X}_{-1}(\Omega)$ consisting of the closure of $\mathscr{C}_{\text {comp }}^{\infty}(\bar{\Omega})=\left\{\left.\varphi\right|_{\Omega}, \varphi \in\right.$ $\mathscr{C}^{\infty}(\bar{\Omega})$ and $\operatorname{supp}(\varphi)$ is bounded $\}$ for the norm

$$
\|\mathbf{v}\|_{\mathbf{X}_{-1}(\Omega)}^{2}:=\|\operatorname{curl}(\mathbf{v})\|_{\mathrm{L}^{2}(\Omega)}^{2}+\|\operatorname{div}(\mathbf{v})\|_{\mathrm{L}^{2}(\Omega)}^{2}+\int_{\Omega} \frac{|\mathbf{v}(\boldsymbol{x})|^{2}}{1+|\boldsymbol{x}|^{2}} d \boldsymbol{x} .
$$

The dual space to $\mathbf{X}_{-1}(\Omega)$ is denoted by $\mathbf{X}_{-1}(\Omega)^{\prime}$ and equipped with the canonical dual norm. According to [20, Lemma A.4], a weighted Poincare-Friedrichs inequality holds in this functional framework: there exists a ball $\mathrm{B} \subset \mathbb{R}^{3}$ and a constant $C>0$ such that $C\|\mathbf{v}\|_{\mathbf{X}_{-1}(\Omega)}^{2} \leq\|\operatorname{curl}(\mathbf{v})\|_{\mathrm{L}^{2}(\Omega)}^{2}+\|\operatorname{div}(\mathbf{v})\|_{\mathrm{L}^{2}(\Omega)}^{2}+\|\mathbf{v}\|_{\mathrm{L}^{2}(\Omega \cap \mathrm{B})}^{2}$ for all $\mathbf{v} \in \mathbf{X}_{\mathrm{loc}}(\Omega)$. Thus $\mathbf{X}_{-1}(\Omega)$ with zero tangential or normal components imposed on $\Gamma$ will supply a framework in which $-\boldsymbol{\Delta}_{\eta}$ is of Fredholm type, see also [33, Sect. 2.5].

Proposition 3.8. Assume that $\Omega \subset \mathbb{R}^{3}$ is a Lipschitz domain such that either $\Omega$ or $\mathbb{R}^{3} \backslash \bar{\Omega}$ is bounded. Denote $\mathbf{X}_{-1, \mathrm{~N}}(\Omega):=\left\{\mathbf{u} \in \mathbf{X}_{-1}(\Omega), \boldsymbol{n} \times(\mathbf{u} \times \boldsymbol{n})=0\right.$ on $\left.\Gamma\right\}$ and $\mathbf{X}_{-1, \mathrm{~T}}(\Omega):=\{\mathbf{u} \in \mathrm{W}(\Omega), \mathbf{u} \cdot \boldsymbol{n}=0$ on $\Gamma\}$. Then for $*=\mathrm{N}$ or $*=\mathrm{T}$, the continuous operator $-\mathcal{D}_{\eta}^{*}: \mathbf{X}_{-1, *}(\Omega) \rightarrow \mathbf{X}_{-1, *}(\Omega)^{\prime}$ defined by the bilinear form $\left\langle-\mathcal{D}_{\eta}^{*} \mathbf{u}, \mathbf{v}\right\rangle:=$ $\int_{\Omega} \operatorname{curl}(\mathbf{u}) \cdot \operatorname{curl}(\mathbf{v})+\eta \operatorname{div}(\mathbf{u}) \operatorname{div}(\mathbf{v}) d \boldsymbol{x} \forall \mathbf{u}, \mathbf{v} \in \mathbf{X}_{-1, *}(\Omega)$ is of Fredholm type.

Of course, the operators $\mathcal{D}_{\eta}^{\mathrm{N}}: \mathbf{X}_{-1, \mathrm{~N}}(\Omega) \rightarrow \mathbf{X}_{-1, \mathrm{~N}}(\Omega)^{\prime}$ and $\mathcal{D}_{\eta}^{\mathrm{T}}: \mathbf{X}_{-1, \mathrm{~T}}(\Omega) \rightarrow \mathbf{X}_{-1, \mathrm{~T}}(\Omega)^{\prime}$ are not one-to-one in general. They admit a priori non-trivial kernels whose dimension is related to the topology of the domain $\Omega$.

3.3.2. Hodge-Helmholtz case. As above "boundary conditions at $\infty$ " can also be imposed through weighted spaces in the case $\kappa \neq 0$ as in [37, Section 4] and [28, Section 3]. However, since we confine ourselves to constant scalar coefficients, we can also invoke the classical strong form of the radiation conditions. Following [17] we require

$$
\begin{aligned}
& \lim _{\rho \rightarrow \infty} \int_{\partial \mathrm{B}_{\rho}}\left|\operatorname{curl}(\mathbf{u}) \times \boldsymbol{n}_{\rho}-\imath \kappa \boldsymbol{n}_{\rho} \times\left(\mathbf{u} \times \boldsymbol{n}_{\rho}\right)\right|^{2} d \sigma_{\rho}=0 \quad \text { if } \quad \mathfrak{I} m\{\kappa\}=0 \\
& \lim _{\rho \rightarrow \infty} \int_{\partial \mathrm{B}_{\rho}}\left|\operatorname{curl}(\mathbf{u}) \times \boldsymbol{n}_{\rho}\right|^{2}+\left|\boldsymbol{n}_{\rho} \times\left(\mathbf{u} \times \boldsymbol{n}_{\rho}\right)\right|^{2} d \sigma_{\rho}=0 \quad \text { if } \quad \Im m\{\kappa\}>0
\end{aligned}
$$

and

$$
\begin{aligned}
& \lim _{\rho \rightarrow \infty} \int_{\partial \mathrm{B}_{\rho}}\left|\sqrt{\eta} \operatorname{div}(\mathbf{u})-\imath \kappa \boldsymbol{n}_{\rho} \cdot \mathbf{u}\right|^{2} d \sigma_{\rho}=0 \quad \text { if } \quad \Im m\{\kappa\}=0 \\
& \lim _{\rho \rightarrow \infty} \int_{\partial \mathrm{B}_{\rho}}|\operatorname{div}(\mathbf{u})|^{2}+\left|\boldsymbol{n}_{\rho} \cdot \mathbf{u}\right|^{2} d \sigma_{\rho}=0 . \quad \text { if } \quad \Im m\{\kappa\}>0 .
\end{aligned}
$$


that can be regarded as a variant of Silver-Müller's radiation condition, a key concept in the analysis of Maxwell's equations in exterior domains. Here $\mathrm{B}_{\rho}$ denotes the ball of center 0 and radius $\rho$, and $\boldsymbol{n}_{\rho}$ refers to the unit normal to $\partial \mathrm{B}_{\rho}$ directed toward the exterior of $\mathrm{B}_{\rho}$. The next result was established in [17].

Corollary 3.9. Assume that $\mathbb{R}^{3} \backslash \bar{\Omega}$ is bounded and $\kappa^{2} \in \mathbb{C}_{+}:=\{z \in \mathbb{C}: \Im m\{z\} \geq$ $0\}, \kappa \neq 0$. Then for any $\mathbf{g} \in \mathcal{H}_{\mathrm{D}}(\Gamma)$ there exists a unique solution to the problem

$$
\left\{\begin{array}{l}
\mathbf{u} \in \mathbf{X}_{\mathrm{loc}}(\Omega) \quad \text { such that } \\
-\boldsymbol{\Delta}_{\eta} \mathbf{u}-\kappa^{2} \mathbf{u}=0 \text { in } \Omega \\
\mathcal{T}_{\mathrm{D}}(\mathbf{u})=\mathbf{g} \text { on } \Gamma \\
\mathbf{u} \text { satisfies (3.12)-(3.13) }
\end{array}\right.
$$

and the solution map is continuous: for any ball $\mathrm{B} \subset \mathbb{R}^{3}$ there exists a constant $C>0$ independent of $\mathbf{g}$ such that $\|\mathbf{u}\|_{\mathbf{X}(\Omega \cap \mathrm{B})} \leq C\|\mathbf{g}\|_{\mathcal{H}_{\mathrm{D}}(\Gamma)}$. Similarly for any $\mathbf{g} \in \mathcal{H}_{\mathrm{N}}(\Gamma)$ there exists a unique solution to the problem

$$
\left\{\begin{array}{l}
\mathbf{u} \in \mathbf{X}_{\mathrm{loc}}(\Omega) \quad \text { such that } \\
-\boldsymbol{\Delta}_{\eta} \mathbf{u}-\kappa^{2} \mathbf{u}=0 \text { in } \Omega \\
\mathcal{T}_{\mathrm{N}}(\mathbf{u})=\mathbf{g} \text { on } \Gamma \\
\mathbf{u} \text { satisfies (3.12)-(3.13) }
\end{array}\right.
$$

Moreover the solution map is continuous: for any ball $\mathrm{B} \subset \mathbb{R}^{3}$ there exists a constant $C>0$ independent of $\mathbf{g}$ such that $\|\mathbf{u}\|_{\mathbf{X}(\Omega \cap \mathrm{B})} \leq C\|\mathbf{g}\|_{\mathcal{H}_{\mathbb{N}}(\Gamma)}$.

\section{Representation formula.}

4.1. Fundamental solution. Fundamental solutions enter boundary integral formulations as a crucial building block. For the Hodge-Helmholtz equation explicit expression can be found in [17] and [11, Chapter 7]. In a much more general context of exterior calculus on a manifold, fundamental solutions are also studied in [24, Sect. 3.1] and [25, Ch. 2].

Lemma 4.1. For $\kappa^{2} \in \mathbb{C}_{+}$the unique smooth function $\mathbb{G}_{\kappa}: \mathbb{R}^{3} \backslash\{0\} \rightarrow \mathbb{C}^{3 \times 3}$ satisfying $-\boldsymbol{\Delta}_{\eta} \mathbb{G}_{\kappa}-\kappa^{2} \mathbb{G}_{\kappa}=\delta_{0} \cdot \operatorname{Id}$ in the sense of distributions and the radiation conditions (3.12) and (3.13) for $\kappa \neq 0$, or the decay conditions implicit in $\mathbf{X}_{-1}(\Omega)$ for $\kappa=0$, is (continuously extended to $\kappa=0$ )

$$
\mathbb{G}_{\kappa}(\boldsymbol{x}):=\mathscr{G}_{\kappa}(\boldsymbol{x}) \mathrm{Id}+\kappa^{-2} \nabla^{2}\left(\mathscr{G}_{\kappa}(\boldsymbol{x})-\mathscr{G}_{\tilde{\kappa}}(\boldsymbol{x})\right),
$$

where $\mathscr{G}_{\kappa}(\boldsymbol{x}):=\exp (\imath \kappa|\boldsymbol{x}|) /(4 \pi|\boldsymbol{x}|)$ is the fundamental solution for the scalar Helmholtz equation and $\tilde{\kappa}:=\kappa / \sqrt{\eta}$.

In the statement of the lemma, $\delta_{0}$ denotes the Dirac distribution centred at $\boldsymbol{x}=0$, Id is the $3 \times 3$ identity matrix, and $\nabla^{2}$ is the Hessian matrix. The equation $-\boldsymbol{\Delta}_{\eta} \mathbb{G}_{\kappa}-$ $\kappa^{2} \mathbb{G}_{\kappa}=\delta_{0} \cdot \operatorname{Id}$ means that, for any constant vector $\mathbf{a} \in \mathbb{C}^{3}$, we have $-\boldsymbol{\Delta}_{\eta}\left(\mathbb{G}_{\kappa}(\boldsymbol{x}) \mathbf{a}\right)$ $\kappa^{2} \mathbb{G}_{\kappa}(\boldsymbol{x}) \mathbf{a}=\delta_{0}(\boldsymbol{x})$ a. In (4.1) observe that, for $\eta=1$, we recover $\mathbb{G}_{\kappa}(\boldsymbol{x})=\mathscr{G}_{\kappa}(\boldsymbol{x})$ Id, necessarily, since the operator $\boldsymbol{\Delta}_{\eta}$ for $\eta=1$ coincides with the classical vector Laplace operator. Appealing to the series expansion of the exponential confirms existence of an entire function $\tilde{g}_{1}: \mathbb{C}^{2} \rightarrow \mathbb{C}$ such that

$$
\mathscr{G}_{\kappa}(\boldsymbol{x})-\mathscr{G}_{\tilde{\kappa}}(\boldsymbol{x})=\frac{\imath \kappa-\imath \tilde{\kappa}}{4 \pi}+\frac{(\imath \kappa)^{2}-(\imath \tilde{\kappa})^{2}}{8 \pi}|\boldsymbol{x}|+\kappa(\kappa|\boldsymbol{x}|)^{2} \tilde{g}_{1}(\kappa,|\boldsymbol{x}|) .
$$


From this we conclude by direct calculation that there exist two other entire functions $\tilde{g}_{2}, \tilde{g}_{3}: \mathbb{C}^{2} \rightarrow \mathbb{C}$ that are analytic in the whole complex plane, and such that

$$
\mathbb{G}_{\kappa}(\boldsymbol{x})=\underbrace{\frac{1}{4 \pi|\boldsymbol{x}|} \operatorname{Id}+\frac{1-\eta}{8 \pi \eta|\boldsymbol{x}|}\left(\operatorname{Id}-\frac{\boldsymbol{x} \cdot \boldsymbol{x}^{T}}{|\boldsymbol{x}|^{2}}\right)}_{:=\mathbb{G}_{0}(\boldsymbol{x})}+\kappa\left(\tilde{g}_{2}(\kappa,|\boldsymbol{x}|) \operatorname{Id}-\tilde{g}_{3}(\kappa,|\boldsymbol{x}|) \frac{\boldsymbol{x} \cdot \boldsymbol{x}^{T}}{|\boldsymbol{x}|^{2}}\right) .
$$

This reveals that $\lim _{\kappa \rightarrow 0} \mathbb{G}_{\kappa}(\boldsymbol{x})=\mathbb{G}_{0}(\boldsymbol{x})$ for all $\boldsymbol{x} \neq 0$. Finally, let us examine what is the effect on $\mathbb{G}_{\kappa}$ of changing the value of $\kappa$. Based on (4.3), we see that there are two entire functions $\tilde{g}_{4}, \tilde{g}_{5}: \mathbb{C}^{3} \rightarrow \mathbb{C}$ such that

$$
\mathbb{G}_{\kappa}(\boldsymbol{x})-\mathbb{G}_{\nu}(\boldsymbol{x})=\tilde{g}_{4}(\kappa, \nu,|\boldsymbol{x}|) \operatorname{Id}+\tilde{g}_{5}(\kappa, \nu,|\boldsymbol{x}|) \frac{\boldsymbol{x} \cdot \boldsymbol{x}^{T}}{|\boldsymbol{x}|^{2}} .
$$

Thus, $(\kappa, \nu) \mapsto \mathscr{G}_{\kappa}(\boldsymbol{x})-\mathscr{G}_{\nu}(\boldsymbol{x})$ is an entire function $\mathbb{C}^{2} \rightarrow \mathrm{L}^{\infty}\left(\mathbb{R}^{3}\right)^{3 \times 3}$.

4.2. Representation by boundary potentials. To derive a representation formula associated to Equation (3.5), we will mimic the approach of M. Costabel in [14]. Let $\mathcal{T}_{\mathrm{D}}^{\prime}: \mathcal{H}_{\mathrm{N}}(\Gamma)=\mathcal{H}_{\mathrm{D}}(\Gamma)^{\prime} \rightarrow \mathbf{X}(\boldsymbol{\Delta}, \Omega)^{\prime}$ refer to the operator adjoint to $\mathcal{T}_{\mathrm{D}}$ i.e. $\left\langle\mathcal{T}_{\mathrm{D}}^{\prime}(\mathbf{p}), \mathbf{v}\right\rangle:=\left\langle\mathbf{p}, \mathcal{T}_{\mathrm{D}}(\mathbf{v})\right\rangle$ for all $\mathbf{p} \in \mathcal{H}_{\mathrm{N}}(\Gamma)$ and all $\mathbf{v} \in \mathbf{X}(\boldsymbol{\Delta}, \Omega)$. We define $\mathcal{T}_{\mathrm{N}}^{\prime}: \mathcal{H}_{\mathrm{D}}(\Gamma) \rightarrow \mathbf{X}(\boldsymbol{\Delta}, \Omega)^{\prime}$ in a similar manner. Note that, since $\mathscr{C}_{\text {comp }}^{\infty}\left(\mathbb{R}^{3}\right) \subset \mathbf{X}(\boldsymbol{\Delta}, \Omega)$, we have $\mathbf{X}(\boldsymbol{\Delta}, \Omega)^{\prime} \subset \mathscr{C}_{\text {comp }}^{\infty}\left(\mathbb{R}^{3}\right)^{\prime}$; in other words, $\mathcal{T}_{\mathrm{D}}^{\prime}(\mathbf{p})$ and $\mathcal{T}_{\mathrm{N}}^{\prime}(\mathbf{q})$ are distributions with compact support, for any $\mathbf{p} \in \mathcal{H}_{\mathrm{N}}(\Gamma)$, and any $\mathbf{q} \in \mathcal{H}_{\mathrm{D}}(\Gamma)$.

Consider any $\mathbf{u} \in \mathrm{L}_{\text {loc }}^{2}\left(\mathbb{R}^{3}\right)^{3}$ such that $\left.\mathbf{u}\right|_{\Omega} \in \mathbf{X}_{\text {loc }}(\Delta, \bar{\Omega})$ and $\left.\mathbf{u}\right|_{\mathbb{R}^{3} \backslash \Omega} \in \mathbf{X}_{\text {loc }}\left(\Delta, \mathbb{R}^{3} \backslash\right.$ $\bar{\Omega})$. Let $\mathbf{f} \in \mathrm{L}_{\text {loc }}^{2}\left(\mathbb{R}^{3}\right)$ be defined by $\left.\mathbf{f}\right|_{\Omega}=\left.\left(-\boldsymbol{\Delta}_{\eta} \mathbf{u}-\kappa^{2} \mathbf{u}\right)\right|_{\Omega}$ and $\left.\mathbf{f}\right|_{\mathbb{R}^{3} \backslash \Omega}=\left(-\boldsymbol{\Delta}_{\eta} \mathbf{u}-\right.$ $\left.\kappa^{2} \mathbf{u}\right)\left.\right|_{\mathbb{R}^{3} \backslash \Omega}$ (restriction here should be understood in the sense of distributions). Let us compute the value of $-\boldsymbol{\Delta}_{\eta} \mathbf{u}-\kappa^{2} \mathbf{u}$ as a distribution in this case. Pick an arbitrary $\boldsymbol{\varphi} \in \mathscr{C}_{\text {comp }}^{\infty}\left(\mathbb{R}^{3}\right)$. Apply Green's formula (3.8) to obtain

$$
\begin{aligned}
\left\langle-\boldsymbol{\Delta}_{\eta} \mathbf{u}-\kappa^{2} \mathbf{u}, \boldsymbol{\varphi}\right\rangle= & -\int_{\Omega} \mathbf{u}\left(\boldsymbol{\Delta}_{\eta} \boldsymbol{\varphi}+\kappa^{2} \boldsymbol{\varphi}\right) d \boldsymbol{x}-\int_{\mathbb{R}^{3} \backslash \bar{\Omega}} \mathbf{u}\left(\boldsymbol{\Delta}_{\eta} \boldsymbol{\varphi}+\kappa^{2} \boldsymbol{\varphi}\right) d \boldsymbol{x} \\
= & \int_{\Omega} \mathbf{f} \boldsymbol{\varphi} d \boldsymbol{x}+\left\langle\left\langle\mathcal{T}_{\mathrm{D}}(\mathbf{u}), \mathcal{T}_{\mathrm{N}}(\boldsymbol{\varphi})\right\rangle-\left\langle\left\langle\mathcal{T}_{\mathrm{D}}(\boldsymbol{\varphi}), \mathcal{T}_{\mathrm{N}}(\mathbf{u})\right\rangle\right\rangle\right. \\
& +\int_{\mathbb{R}^{3} \backslash \bar{\Omega}} \mathbf{f} \boldsymbol{\varphi} d \boldsymbol{x}-\left\langle\left\langle\mathcal{T}_{\mathrm{D}, c}(\mathbf{u}), \mathcal{T}_{\mathrm{N}}(\boldsymbol{\varphi})\right\rangle+\left\langle\left\langle\mathcal{T}_{\mathrm{D}}(\boldsymbol{\varphi}), \mathcal{T}_{\mathrm{N}, c}(\mathbf{u})\right\rangle\right\rangle\right. \\
= & \int_{\mathbb{R}^{3}} \mathbf{f} \boldsymbol{\varphi} d \boldsymbol{x}+\left\langle\left\langle\left[\mathcal{T}_{\mathrm{D}}(\mathbf{u})\right], \mathcal{T}_{\mathrm{N}}(\boldsymbol{\varphi})\right\rangle-\left\langle\left\langle\mathcal{T}_{\mathrm{D}}(\boldsymbol{\varphi}),\left[\mathcal{T}_{\mathrm{N}}(\mathbf{u})\right]\right\rangle\right.\right.
\end{aligned}
$$

Above we used the fact that, since $\varphi$ is smooth across the boundary $\Gamma$, we have $\mathcal{T}_{\mathrm{D}, c}(\varphi)=\mathcal{T}_{\mathrm{D}}(\varphi)$ and $\mathcal{T}_{\mathrm{N}, c}(\varphi)=\mathcal{T}_{\mathrm{N}}(\varphi)$. Since the calculus above holds for any $\varphi \epsilon$ $\mathscr{C}_{\text {comp }}^{\infty}\left(\mathbb{R}^{3}\right)$ we finally conclude that, in the sense of distributions, we have

$$
-\boldsymbol{\Delta}_{\eta} \mathbf{u}-\kappa^{2} \mathbf{u}=\mathbf{f}+\mathcal{T}_{\mathrm{N}}^{\prime} \cdot\left[\mathcal{T}_{\mathrm{D}}(\mathbf{u})\right]-\mathcal{T}_{\mathrm{D}}^{\prime} \cdot\left[\mathcal{T}_{\mathrm{N}}(\mathbf{u})\right]
$$

This leads to an expression of $\mathbf{u}$ by multiplying on the left by the convolution operator associated to the (outgoing) Green tensor $\mathbb{G}_{\kappa}$ given by (4.1),

$$
\begin{aligned}
& \mathbf{u}=\mathbb{G}_{\kappa} * \mathbf{f}+\mathcal{S} \mathcal{L}_{\kappa} \cdot\left[\mathcal{T}_{\mathrm{N}}(\mathbf{u})\right]+\mathcal{D} \mathcal{L}_{\kappa} \cdot\left[\mathcal{T}_{\mathrm{D}}(\mathbf{u})\right] \\
& \text { where } \quad \mathcal{S} \mathcal{L}_{\kappa}(\mathfrak{p})=-\mathbb{G}_{\kappa} * \mathcal{T}_{\mathrm{D}}^{\prime}(\mathfrak{p}) \text { for } \mathfrak{p} \in \mathcal{H}_{\mathrm{N}}(\Gamma) \text {, } \\
& \mathcal{D} \mathcal{L}_{\kappa}(\mathfrak{v})=+\mathbb{G}_{\kappa} * \mathcal{T}_{N}^{\prime}(\mathfrak{v}) \quad \text { for } \mathfrak{v} \in \mathcal{H}_{\mathrm{D}}(\Gamma) \text {. }
\end{aligned}
$$


The convolutions above should be understood in the sense of ( $\mathbb{C}^{3}$-valued) distributions. We will call $\mathcal{S} \mathcal{L}_{\kappa}\left(\right.$ resp. $\left.\mathcal{D} \mathcal{L}_{\kappa}\right)$ the Hodge-Helmholtz single (resp. double) layer potential by analogy with the classical potential operators for the scalar Helmholtz equation. To derive their integral representations, note that for $\boldsymbol{x} \notin \Gamma$ the $j$-th component of the potentials at $\boldsymbol{x}$ is

$$
\begin{aligned}
& \boldsymbol{e}_{j} \cdot \mathcal{S} \mathcal{L}_{\kappa}(\mathfrak{p})(\boldsymbol{x})=-\left\langle\left\langle\mathfrak{p}, \mathcal{T}_{\mathrm{D}}\left(\mathbb{G}_{\kappa}(\boldsymbol{x}-\cdot) \boldsymbol{e}_{j}\right)\right\rangle\right\rangle \\
& \boldsymbol{e}_{j} \cdot \mathcal{D} \mathcal{L}_{\kappa}(\mathfrak{v})(\boldsymbol{x})=\left\langle\left\langle\mathcal{T}_{\mathrm{N}}\left(\mathbb{G}_{\kappa}(\boldsymbol{x}-\cdot) \boldsymbol{e}_{j}\right), \mathfrak{v}\right\rangle\right\rangle
\end{aligned}
$$

where $\boldsymbol{e}_{j}$ stands for the $j$-th Cartesian unit vector, $j=1,2,3$.

First, we establish an integral representation formula for the Hodge-Helmholtz single layer potential. We write $\mathfrak{p}=(\mathbf{p}, \alpha) \in \mathcal{H}_{\mathrm{N}}(\Gamma)=\mathbf{H}^{-\frac{1}{2}}(\operatorname{div}, \Gamma) \times \mathrm{H}^{-\frac{1}{2}}(\Gamma)$ and split the duality pairings into their components, which yields

$$
\boldsymbol{e}_{j} \cdot \mathcal{S} \mathcal{L}_{\kappa}(\mathfrak{p})(\boldsymbol{x})=-\left\langle\mathbf{p}, \gamma_{\mathrm{T}}\left(\mathbb{G}_{\kappa}(\boldsymbol{x}-\cdot) \boldsymbol{e}_{j}\right)\right\rangle-\left\langle\alpha, \gamma_{\mathrm{D}}\left(\mathbb{G}_{\kappa}(\boldsymbol{x}-\cdot) \boldsymbol{e}_{j}\right)\right\rangle .
$$

For the sake of brevity we set $\widetilde{\mathscr{G}}_{\kappa}(\boldsymbol{x}):=\kappa^{-2}\left(\mathscr{G}_{\kappa}(\boldsymbol{x})-\mathscr{G}_{\tilde{\kappa}}(\boldsymbol{x})\right)$ using the notations from Lemma 4.1. Then, denoting $\partial_{j}:=\boldsymbol{e}_{j} \cdot \nabla$, the two terms on the right-hand side of (4.8) evaluate to

$$
\begin{aligned}
\left\langle\mathbf{p}, \gamma_{\mathrm{T}}\left(\mathbb{G}_{\kappa}(\boldsymbol{x}-\cdot) \boldsymbol{e}_{j}\right)\right\rangle & =\int_{\Gamma} \mathbf{p}(\boldsymbol{y}) \cdot\left(\mathscr{G}_{\kappa}(\boldsymbol{x}-\boldsymbol{y}) \boldsymbol{e}_{j}+\left(\nabla \partial_{j} \widetilde{\mathscr{G}}_{\kappa}\right)(\boldsymbol{x}-\boldsymbol{y})\right) d \sigma(\boldsymbol{y}), \\
\left\langle\alpha, \gamma_{\mathrm{D}}\left(\mathbb{G}_{\kappa}(\boldsymbol{x}-\cdot) \boldsymbol{e}_{j}\right)\right\rangle & =\eta \int_{\Gamma} \alpha(\boldsymbol{y}) \operatorname{div} \boldsymbol{y}\left(\mathscr{G}_{\kappa}(\boldsymbol{x}-\boldsymbol{y}) \boldsymbol{e}_{j}+\left(\nabla \partial_{j} \widetilde{\mathscr{G}}_{\kappa}\right)(\boldsymbol{x}-\boldsymbol{y})\right) d \sigma(\boldsymbol{y}) \\
& =-\eta \int_{\Gamma} \alpha(\boldsymbol{y})\left(\partial_{j} \mathscr{G}_{\kappa}(\boldsymbol{x}-\boldsymbol{y})+\left(\partial_{j} \Delta \widetilde{\mathscr{G}}_{\kappa}\right)(\boldsymbol{x}-\boldsymbol{y})\right) d \sigma(\boldsymbol{y}) \\
& =-\int_{\Gamma}\left(\partial_{j} \mathscr{G}_{\widetilde{\kappa}}\right)(\boldsymbol{x}-\boldsymbol{y}) \alpha(\boldsymbol{y}) d \sigma(\boldsymbol{y})
\end{aligned}
$$

where we used the identity $\Delta \widetilde{\mathscr{G}}_{\kappa}(\boldsymbol{x})=-\mathscr{G}_{\kappa}(\boldsymbol{x})+\eta^{-1} \mathscr{\mathscr { G }}_{\widetilde{\kappa}}(\boldsymbol{x})$ for $\boldsymbol{x} \neq 0$. Collecting vector components and integrating by parts on $\Gamma$ we arrive at

$$
\begin{aligned}
\mathcal{S L}_{\kappa}(\mathfrak{p})(\boldsymbol{x}):= & -\int_{\Gamma} \mathscr{G}_{\kappa}(\boldsymbol{x}-\boldsymbol{y}) \mathbf{p}(\boldsymbol{y}) d \sigma(\boldsymbol{y}) \\
& -\int_{\Gamma}\left(\nabla \widetilde{\mathscr{G}}_{\kappa}\right)(\boldsymbol{x}-\boldsymbol{y}) \operatorname{div}_{\Gamma} \mathbf{p}(\boldsymbol{y}) d \sigma(\boldsymbol{y}) \\
& +\nabla_{\boldsymbol{x}} \int_{\Gamma} \mathscr{G}_{\widetilde{\kappa}}(\boldsymbol{x}-\boldsymbol{y}) \alpha(\boldsymbol{y}) d \sigma(\boldsymbol{y}), \quad \boldsymbol{x} \notin \Gamma .
\end{aligned}
$$

Remark 4.2. Generically the integrals $\int_{\Gamma} \ldots d \sigma(\boldsymbol{y})$ in (4.9) have to be read as duality pairings. However, all kernels are integrable on $\Gamma$. Therefore, the integrals can be understood as classical improper integrals provided that $\mathbf{p}, \alpha$, and $\operatorname{div}_{\Gamma} \mathbf{p}$ belong to $\mathrm{L}^{\infty}(\Gamma)$.

A similar integral formula can be derived for the Hodge-Helmholtz double layer potential $\mathcal{D} \mathcal{L}_{\kappa}$ starting from

$$
\left\langle\mathcal{T}_{\mathrm{N}}\left(\mathbb{G}_{\kappa}(\boldsymbol{x}-\cdot) \boldsymbol{e}_{j}\right), \mathfrak{v}\right\rangle=\left\langle\gamma_{\mathrm{R}}\left(\mathbb{G}_{\kappa}(\boldsymbol{x}-\cdot) \boldsymbol{e}_{j}\right), \mathbf{q}\right\rangle+\left\langle\gamma_{\mathrm{N}}\left(\mathbb{G}_{\kappa}(\boldsymbol{x}-\cdot) \boldsymbol{e}_{j}\right), \beta\right\rangle,
$$

where $\mathfrak{v}=(\mathbf{q}, \beta) \in \mathcal{H}_{\mathrm{D}}(\Gamma)=\mathbf{H}^{-\frac{1}{2}}(\operatorname{curl}, \Gamma) \times \mathrm{H}^{+\frac{1}{2}}(\Gamma)$. Next, we use $\operatorname{curl}\left(\mathbb{G}_{\kappa}(\boldsymbol{x}) \boldsymbol{e}_{j}\right)=$ $\operatorname{curl}\left(\mathscr{G}_{\kappa}(\boldsymbol{x}) \boldsymbol{e}_{j}-\nabla^{2} \widetilde{\mathscr{G}}_{\kappa}(\boldsymbol{x}) \boldsymbol{e}_{j}\right)=\left(\nabla \mathscr{G}_{\kappa}\right)(\boldsymbol{x}) \times \boldsymbol{e}_{j}$, which is applied to manipulate the first 
term in (4.10). By means of the identity $\mathbf{a} \cdot(\mathbf{b} \times \mathbf{c})=\mathbf{c} \cdot(\mathbf{a} \times \mathbf{b}), \mathbf{a}, \mathbf{b}, \mathbf{c} \in \mathbb{R}^{3}$, we thus obtain

$$
\begin{aligned}
\left\langle\gamma_{\mathrm{R}}\left(\mathbb{G}_{\kappa}(\boldsymbol{x}-\cdot) \boldsymbol{e}_{j}\right), \mathbf{q}\right\rangle & =\int_{\Gamma} \mathbf{q}(\boldsymbol{y}) \cdot \boldsymbol{n}(\boldsymbol{y}) \times \operatorname{curl}_{\boldsymbol{y}}\left(\mathbb{G}_{\kappa}(\boldsymbol{x}-\boldsymbol{y}) \boldsymbol{e}_{j}\right) d \sigma(\boldsymbol{y}) \\
& =-\int_{\Gamma}\left(\left(\nabla^{\mathscr{G}_{\kappa}}\right)(\boldsymbol{x}-\boldsymbol{y}) \times \boldsymbol{e}_{j}\right) \cdot(\mathbf{q}(\boldsymbol{y}) \times \boldsymbol{n}(\boldsymbol{y})) d \sigma(\boldsymbol{y}) \\
& =-\boldsymbol{e}_{j} \cdot \int_{\Gamma}(\mathbf{q}(\boldsymbol{y}) \times \boldsymbol{n}(\boldsymbol{y})) \times\left(\nabla \mathscr{G}_{\kappa}\right)(\boldsymbol{x}-\boldsymbol{y}) d \sigma(\boldsymbol{y}) \\
& =\boldsymbol{e}_{j} \cdot \int_{\Gamma} \operatorname{curl}_{\boldsymbol{x}}\left(\mathscr{G}_{\kappa}(\boldsymbol{x}-\boldsymbol{y}) \mathbf{q}(\boldsymbol{y}) \times \boldsymbol{n}(\boldsymbol{y})\right) d \sigma(\boldsymbol{y})
\end{aligned}
$$

which finally yields the vector identity

$$
\left(\left\langle\gamma_{\mathrm{R}}\left(\mathbb{G}_{\kappa}(\boldsymbol{x}-\cdot)\right), \mathbf{q}\right\rangle\right)_{j=1}^{3}=\operatorname{curl}_{\boldsymbol{x}} \int_{\Gamma} \mathscr{G}_{\kappa}(\boldsymbol{x}-\boldsymbol{y})(\mathbf{q}(\boldsymbol{y}) \times \boldsymbol{n}(\boldsymbol{y})) d \sigma(\boldsymbol{y}) .
$$

The second term in (4.10) can be transformed to

$$
\begin{aligned}
\left\langle\gamma_{\mathrm{N}}\left(\mathbb{G}_{\kappa}(\boldsymbol{x}-\cdot) \boldsymbol{e}_{j}\right), \beta\right\rangle & =\int_{\Gamma} \beta(\boldsymbol{y})\left(\boldsymbol{n}(\boldsymbol{y}) \cdot \mathbb{G}(\boldsymbol{x}-\boldsymbol{y}) \boldsymbol{e}_{j}\right) d \sigma(\boldsymbol{y}) \\
& =\int_{\Gamma} \mathscr{G}_{\kappa}(\boldsymbol{x}-\boldsymbol{y}) \beta(\boldsymbol{y}) \boldsymbol{n}(\boldsymbol{y}) \cdot \boldsymbol{e}_{j}+\beta(\boldsymbol{y})\left(\partial_{j} \nabla \widetilde{\mathscr{G}}_{\kappa}\right)(\boldsymbol{x}-\boldsymbol{y}) \cdot \boldsymbol{n}(\boldsymbol{y}) d \sigma(\boldsymbol{y}) .
\end{aligned}
$$

Again, assembling vector components we get

$$
\begin{aligned}
\left(\left\langle\gamma_{\mathrm{N}}\left(\mathbb{G}_{\kappa}(\boldsymbol{x}-\cdot) \boldsymbol{e}_{j}\right), \beta\right\rangle\right)_{j=1}^{3}= & \int_{\Gamma} \mathscr{G}_{\kappa}(\boldsymbol{x}-\boldsymbol{y}) \boldsymbol{n}(\boldsymbol{y}) \beta(\boldsymbol{y}) d \sigma(\boldsymbol{y})- \\
& \nabla_{\boldsymbol{x}} \int_{\Gamma} \boldsymbol{n}(\boldsymbol{y}) \cdot\left(\nabla \widetilde{\mathscr{G}}_{\kappa}\right)(\boldsymbol{x}-\boldsymbol{y}) \beta(\boldsymbol{y}) d \sigma(\boldsymbol{y}) .
\end{aligned}
$$

We end up with

$$
\begin{aligned}
& \hline \mathcal{D L} \mathcal{L}_{\kappa}(\mathfrak{q})(\boldsymbol{x}):= \operatorname{curl}_{\boldsymbol{x}} \int_{\Gamma} \mathscr{G}_{\kappa}(\boldsymbol{x}-\boldsymbol{y}) \mathbf{q}(\boldsymbol{y}) \times \boldsymbol{n}(\boldsymbol{y}) d \sigma(\boldsymbol{y})+ \\
& \int_{\Gamma} \mathscr{G}_{\kappa}(\boldsymbol{x}-\boldsymbol{y}) \boldsymbol{n}(\boldsymbol{y}) \beta(\boldsymbol{y}) d \sigma(\boldsymbol{y})- \\
& \nabla_{\boldsymbol{x}} \int_{\Gamma} \boldsymbol{n}(\boldsymbol{y}) \cdot\left(\nabla \widetilde{\mathscr{G}}_{\kappa}\right)(\boldsymbol{x}-\boldsymbol{y}) \beta(\boldsymbol{y}) d \sigma(\boldsymbol{y}), \quad \boldsymbol{x} \notin \Gamma . \\
& \hline
\end{aligned}
$$

Remark 4.3. Also in the case of (4.11) the integrability of the kernels permits us to regard the integrals as classical improper integrals provided that $\mathbf{q}, \beta \in \mathrm{L}^{\infty}(\Gamma)$.

5. Properties of potentials. Now we examine the continuity properties and jump relations for the potentials given by the expressions (4.9) and (4.11) above. We first scrutinize their building blocks. We will repeatedly rely on continuity properties that were established for more classical potential operators in [14] (for scalar-valued potentials) and in [9] (for vector-valued potentials).

5.1. Scalar single-layer potential. Before studying in detail the properties of the operators (4.9)-(4.11), we recall the classical single layer operator $\psi_{\nu}$ that maps any $\alpha \in \mathrm{H}^{-1 / 2}(\Gamma)$ to the function $\left(\nu \in \mathbb{C}_{+}\right)$

$$
\psi_{\nu}(\alpha)(\boldsymbol{x}):=\int_{\Gamma} \alpha(\boldsymbol{y}) \mathscr{G}_{\nu}(\boldsymbol{x}-\boldsymbol{y}) d \sigma(\boldsymbol{y}), \quad \boldsymbol{x} \in \mathbb{R}^{3} \backslash \Gamma .
$$

We know that $\psi$ maps continuously $\mathrm{H}^{-1 / 2}(\Gamma)$ into $\mathrm{H}_{\mathrm{loc}}^{1}(\Delta, \bar{\Omega}) \times \mathrm{H}_{\mathrm{loc}}^{1}\left(\Delta, \mathbb{R}^{3} \backslash \Omega\right)$, see [32, Sect. 3.1.2]. Besides, we have $\Delta \psi_{\nu}(\alpha)=-\nu^{2} \psi_{\nu}(\alpha)$ and $\operatorname{curl}\left(\nabla \psi_{\nu}(\alpha)\right)=0$ both 
in $\Omega$ and in $\mathbb{R}^{3} \backslash \bar{\Omega}$. We conclude that $\nabla \psi_{\nu}$ maps continuously $\mathrm{H}^{-1 / 2}(\Gamma)$ both into $\mathbf{X}_{\mathrm{loc}}(\boldsymbol{\Delta}, \Omega)$ and $\mathbf{X}_{\mathrm{loc}}\left(\boldsymbol{\Delta}, \mathbb{R}^{3} \backslash \bar{\Omega}\right)$.

In addition, we know that $\psi_{\nu}(\alpha) \in \mathrm{H}_{\mathrm{loc}}^{1}\left(\mathbb{R}^{3}\right)$ for any $\alpha \in \mathrm{H}^{-1 / 2}(\Gamma)$. Classical jump relations for this potential operator read $\left[\tau_{\mathrm{D}}\right] \cdot \psi_{\nu}(\alpha)=0$ and $\left[\tau_{\mathrm{N}}\right] \cdot \psi_{\nu}(\alpha)=\alpha[32$, Sect. 3.3.1]. Since $\operatorname{div}\left(\nabla \psi_{\nu}(\alpha)\right)=-\nu^{2} \psi_{\nu}(\alpha)$, we conclude that

$$
\begin{array}{ll}
{\left[\gamma_{\mathrm{D}}\right] \cdot \nabla \psi_{\nu}(\alpha)=0,} & {\left[\gamma_{\mathrm{T}}\right] \cdot \nabla \psi_{\nu}(\alpha)=0,} \\
{\left[\gamma_{\mathrm{R}}\right] \cdot \nabla \psi_{\nu}(\alpha)=0,} & {\left[\gamma_{\mathrm{N}}\right] \cdot \nabla \psi_{\nu}(\alpha)=\alpha .}
\end{array}
$$

5.2. Vector single layer potential. Similarly, we may consider a vector version of the single-layer potential denoted $\Psi_{\nu}$ that maps any tangential $\mathbf{p} \in \mathbf{H}^{-1 / 2}\left(\operatorname{div}_{\Gamma}, \Gamma\right)$ to the function

$$
\Psi_{\nu}(\mathbf{p})(\boldsymbol{x}):=\int_{\Gamma} \mathbf{p}(\boldsymbol{y}) \mathscr{G}_{\nu}(\boldsymbol{x}-\boldsymbol{y}) d \sigma(\boldsymbol{y}) .
$$

We know from [7] that $\Psi_{\nu}$ continuously maps $\mathbf{H}_{\mathrm{R}}^{-1 / 2}(\Gamma)$ into $\mathrm{H}_{\mathrm{loc}}^{1}\left(\mathbb{R}^{3}\right)^{3}$, which immediately implies $\left[\gamma_{\mathrm{T}}\right] \cdot \Psi(\mathbf{p})=0$ and $\left[\gamma_{\mathrm{N}}\right] \cdot \Psi(\mathbf{p})=0$ for all $\mathbf{p} \in \mathbf{H}^{-1 / 2}(\operatorname{div}, \Gamma)$. Moreover we know from [22, Lemma 2.3] that $\operatorname{div}\left(\Psi_{\nu}(\mathbf{p})\right)=\psi_{\nu}\left(\operatorname{div}_{\Gamma} \mathbf{p}\right)$ so that $\left[\gamma_{\mathrm{D}}\right] \cdot \Psi_{\nu}(\mathbf{p})=0$. Finally the jump formulas from $[9$, Thm. 7$]$ imply that $\left[\gamma_{\mathrm{R}}\right] \cdot \Psi_{\nu}(\mathbf{p})=-\mathbf{p}$. To sum up we have

$$
\begin{array}{ll}
{\left[\gamma_{\mathrm{D}}\right] \cdot \Psi_{\nu}(\mathbf{p})=0,} & {\left[\gamma_{\mathrm{T}}\right] \cdot \Psi_{\nu}(\mathbf{p})=0,} \\
{\left[\gamma_{\mathrm{R}}\right] \cdot \Psi_{\nu}(\mathbf{p})=-\mathbf{p},} & {\left[\gamma_{\mathrm{N}}\right] \cdot \Psi_{\nu}(\mathbf{p})=0 .}
\end{array}
$$

Since $\operatorname{div}\left(\Psi_{\nu}(\mathbf{p})\right)=\psi_{\nu}\left(\operatorname{div}_{\Gamma} \mathbf{p}\right)$ and $\psi_{\nu}$ continuously maps $\mathrm{H}^{-1 / 2}(\Gamma)$ into $\mathrm{H}_{\mathrm{loc}}^{1}(\Delta, \bar{\Omega}) \times$ $\mathrm{H}_{\text {loc }}^{1}\left(\Delta, \mathbb{R}^{3} \backslash \Omega\right)$, we conclude that $\Psi_{\nu}$ continuously maps $\mathbf{H}^{-1 / 2}(\operatorname{div}, \Gamma)$ into $\mathbf{H}_{\mathrm{loc}}(\nabla \operatorname{div}, \Omega) \times$ $\mathbf{H}_{\text {loc }}\left(\nabla \operatorname{div}, \mathbb{R}^{3} \backslash \bar{\Omega}\right)$. In addition $\Delta \Psi_{\nu}(\mathbf{p})=-\nu^{2} \Psi_{\nu}(\mathbf{p})$ in $\Omega$ (resp. $\left.\mathbb{R}^{3} \backslash \bar{\Omega}\right)$, and since $-\boldsymbol{\Delta}=\operatorname{curl}(\operatorname{curl} \cdot)-\nabla(\operatorname{div} \cdot)$ we conclude that $\Psi_{\nu}$ also continuously maps $\mathbf{H}^{-1 / 2}(\operatorname{div}, \Gamma)$ into $\mathbf{H}_{\mathrm{loc}}\left(\mathbf{c u r l}^{2}, \Omega\right) \times \mathbf{H}_{\mathrm{loc}}\left(\mathbf{c u r l}^{2}, \mathbb{R}^{3} \backslash \bar{\Omega}\right)$. In conclusion, we see that $\Psi_{\nu}$ continuously maps $\mathbf{H}^{-1 / 2}(\operatorname{div}, \Gamma)$ into $\mathbf{X}_{\text {loc }}(\boldsymbol{\Delta}, \Omega)\left(\operatorname{resp} . \mathbf{X}_{\text {loc }}\left(\boldsymbol{\Delta}, \mathbb{R}^{3} \backslash \bar{\Omega}\right)\right)$.

5.3. Maxwell double layer potential. Finally let us consider the operator $\mathbf{p} \mapsto \operatorname{curl} \Psi_{\nu}(\mathbf{p})$ the so-called Maxwell double layer potential [9, (28)]. It is well established that curl $\Psi_{\nu}$ continuously maps $\mathbf{H}^{-1 / 2}(\operatorname{div}, \Gamma)$ into $\mathbf{X}_{\text {loc }}(\boldsymbol{\Delta}, \Omega)\left(\operatorname{resp} . \mathbf{X}_{\text {loc }}\left(\boldsymbol{\Delta}, \mathbb{R}^{3} \backslash\right.\right.$ $\bar{\Omega})$ ), see [9, Thm. 5].

As regards the behavior of $\operatorname{curl} \Psi_{\nu}$ across $\Gamma$, first note that, obviously, $\left[\gamma_{\mathrm{D}}\right]$. $\operatorname{curl} \Psi_{\kappa}=0$. In addition observe that $\gamma_{\mathrm{R}}(\mathbf{p}) \times \boldsymbol{n}=\gamma_{\mathrm{T}} \cdot \operatorname{curl}(\mathbf{p})$ so that, according to (5.3), we have $\left[\gamma_{\mathrm{T}}\right] \cdot \operatorname{curl} \Psi_{\nu}(\mathbf{p})=\boldsymbol{n} \times \mathbf{p}$. Next, we have $\left[\gamma_{\mathrm{N}}\right] \cdot \operatorname{curl} \Psi_{\nu}(\mathbf{p})=\operatorname{curl}_{\Gamma}\left(\left[\gamma_{\mathrm{T}}\right]\right.$. $\left.\Psi_{\nu}(\mathbf{p})\right)=0$. Finally we have $\left[\gamma_{\mathrm{R}}\right] \cdot \operatorname{curl} \Psi_{\nu}(\mathbf{p})=0$, which is a well established result of potential theory related to Maxwell's equation [10]. To summarize we have

$$
\begin{array}{ll}
{\left[\gamma_{\mathrm{D}}\right] \cdot \operatorname{curl} \Psi_{\nu}(\mathbf{p})=0,} & {\left[\gamma_{\mathrm{T}}\right] \cdot \operatorname{curl} \Psi_{\nu}(\mathbf{p})=\boldsymbol{n} \times \mathbf{p},} \\
{\left[\gamma_{\mathrm{R}}\right] \cdot \operatorname{curl} \Psi_{\nu}(\mathbf{p})=0,} & {\left[\gamma_{\mathrm{N}}\right] \cdot \operatorname{curl} \Psi_{\nu}(\mathbf{p})=0 .}
\end{array}
$$

5.4. Normal vector single-layer potential. In this paragraph we examine the properties of the operator $\Upsilon_{\nu}:=\mathbb{G}_{\nu} * \gamma_{\mathrm{N}}^{\prime}$ also explicitely described by the following expression

$$
\Upsilon_{\nu}(\alpha)(\boldsymbol{x}):=\int_{\Gamma} \alpha(\boldsymbol{y}) \mathbb{G}_{\nu}(\boldsymbol{x}-\boldsymbol{y}) \cdot \boldsymbol{n}(\boldsymbol{y}) d \sigma(\boldsymbol{y}),
$$

which amounts to a vector single layer potential supplied with a purely normal vector field. From the explicit definition (4.1), we see that the kernel $\mathbb{G}_{\nu}(\boldsymbol{x})$ admits a 
pseudo-homogeneous expansion of degree -1 (see [19, Def. 7.1.1]) so that the associated potential $\mathbf{u} \mapsto \int_{\mathbb{R}^{3}} \mathbb{G}_{\nu}(\boldsymbol{x}-\boldsymbol{y}) \cdot \mathbf{u}(\boldsymbol{y}) d \sigma(\boldsymbol{y})$ is a (vector) pseudo-differential operator of order -2 . Since $\gamma_{\mathrm{N}}$ maps continuously $\mathrm{H}_{\text {loc }}\left(\operatorname{div}, \mathbb{R}^{3}\right)$ into $\mathrm{H}^{-1 / 2}(\Gamma)$, the transpose operator $\gamma_{\mathrm{N}}^{\prime}: \mathrm{H}^{+1 / 2}(\Gamma) \rightarrow \mathrm{H}_{\text {loc }}\left(\operatorname{div}, \mathbb{R}^{3}\right)^{\prime} \subset \mathrm{H}_{\text {comp }}^{-1}\left(\mathbb{R}^{3}\right)$ is continuous, so that $\Upsilon_{\nu}$ maps continuously $\mathrm{H}^{+1 / 2}(\Gamma)$ into $\mathrm{H}_{\mathrm{loc}}^{1}\left(\mathbb{R}^{3}\right)^{3}$.

By construction we have $\boldsymbol{\Delta}_{\eta} \Upsilon_{\nu}(\alpha)=-\nu^{2} \Upsilon_{\nu}(\alpha)$ in $\Omega$ (resp. in $\left.\mathbb{R}^{3} \backslash \bar{\Omega}\right)$ for any $\alpha \epsilon$ $\mathrm{H}^{1 / 2}(\Gamma)$. Besides $\operatorname{curl} \Upsilon_{\nu}(\alpha)=\operatorname{curl} \Psi_{\nu}(\alpha \boldsymbol{n})$ so that $\Upsilon_{\nu}$ maps continuously $\mathrm{H}^{1 / 2}(\Gamma)$ into $\mathbf{H}\left(\mathbf{c u r l}^{2}, \Omega\right)$ according to $\S 5.2$ above. To sum up then, $\Upsilon_{\nu}$ continuously maps $\mathrm{H}^{1 / 2}(\Gamma)$ into $\mathbf{X}_{\text {loc }}(\boldsymbol{\Delta}, \Omega)$ (resp. in $\mathbf{X}_{\text {loc }}\left(\boldsymbol{\Delta}, \mathbb{R}^{3} \backslash \bar{\Omega}\right)$ ). From the discussion above, we also conclude that $\left[\gamma_{\mathrm{N}}\right] \cdot \Upsilon_{\nu}=0$ and $\left[\gamma_{\mathrm{T}}\right] \cdot \Upsilon_{\nu}=0$.

Now observe that for any constant vector $\mathbf{a} \in \mathbb{C}^{3}$ we have $\operatorname{div}\left(\mathbb{G}_{\nu}(\boldsymbol{x}) \cdot \mathbf{a}\right)=\eta^{-1} \mathbf{a}$. $\nabla \mathscr{G}_{\tilde{\nu}}(\boldsymbol{x})$ where $\tilde{\nu}=\nu / \sqrt{\eta}$. Based on this observation, for any $\alpha \in \mathrm{H}^{1 / 2}(\Gamma)$, the following identity is easily derived

$$
\operatorname{div}\left(\Upsilon_{\nu}(\alpha)(\boldsymbol{x})\right)=\frac{1}{\eta} \int_{\Gamma} \alpha(\boldsymbol{y}) \boldsymbol{n}(\boldsymbol{y}) \cdot\left(\nabla \mathscr{G}_{\tilde{\nu}}\right)(\boldsymbol{x}-\boldsymbol{y}) d \sigma(\boldsymbol{y}) \quad \forall \boldsymbol{x} \in \mathbb{R}^{3} \backslash \Gamma .
$$

In the expression above we recognize the well-known scalar double-layer potential arising e.g. in the analysis of acoustic scattering problems (with wave number $\tilde{\nu}$ ), see $[32, \S 3.1]$. Let $\mathrm{DL}_{\tilde{\nu}}:=\eta \operatorname{div}\left(\Upsilon_{\nu}(\cdot)\right): \mathrm{H}^{1 / 2}(\Gamma) \rightarrow \mathrm{H}_{\mathrm{loc}}^{1}(\Delta, \bar{\Omega}) \times \mathrm{H}_{\mathrm{loc}}^{1}\left(\Delta, \mathbb{R}^{3} \backslash \Omega\right)$ refer to this continuous operator. From the standard jump properties of the double layer potential, we deduce $\left[\gamma_{\mathrm{D}}\right] \cdot \Upsilon_{\nu}(\alpha)=\eta^{-1}\left[\tau_{\mathrm{D}}\right] \cdot \mathrm{DL}_{\tilde{\nu}}(\alpha)=\alpha / \eta$.

General jump relations for the curl of vector single layer potentials are given in [13, Thm. 6.13] and [31, Sect. 4] for smooth domains, and in [25, (6.8)] for Lipschitz domains and surface vector fields in $L^{p}(\partial \Omega)^{3}, 1<p<\infty$. They tell us that the tangential components of $\operatorname{curl} \Upsilon_{\nu}(\alpha)$ will not jump across $\partial \Omega$. To summarize, we have established the following jump relations

$$
\begin{array}{ll}
{\left[\gamma_{\mathrm{D}}\right] \cdot \Upsilon_{\nu}(\alpha)=\alpha / \eta,} & {\left[\gamma_{\mathrm{T}}\right] \cdot \Upsilon_{\nu}(\alpha)=0,} \\
{\left[\gamma_{\mathrm{R}}\right] \cdot \Upsilon_{\nu}(\alpha)=0,} & {\left[\gamma_{\mathrm{N}}\right] \cdot \Upsilon_{\nu}(\alpha)=0 .}
\end{array}
$$

5.5. Regular potential. For $\eta \neq 1$ the potential

$$
\widetilde{\psi}_{\nu}(\alpha)(\boldsymbol{x}):=\int_{\Gamma} \widetilde{\mathscr{G}}_{\nu}(\boldsymbol{x}-\boldsymbol{y}) \alpha(\boldsymbol{y}) d \sigma(\boldsymbol{y}) \quad x \in \mathbb{R}^{3} \backslash\{0\},
$$

with $\widetilde{\mathscr{G}}_{\nu}:=\nu^{-2}\left(\mathscr{G}_{\nu}-\mathscr{G}_{\tilde{\nu}}\right)$, occur in both the vector Helmholtz single and double layer potential. By (4.2), the kernel $\widetilde{\mathscr{G}}_{\kappa}$ is the sum of a constant and a pseudo-homogeneous kernel of degree 1 in the sense of [19, Def .7.1.1], see, e.g., Remark 3.1.3 and Lemma 3.9 .8 in [32] for further details on this classical point. Consequently, $\widetilde{\psi}_{\nu}$ provides a continuous mapping $\mathrm{H}^{-1 / 2}(\Gamma) \rightarrow \mathrm{H}_{\mathrm{loc}}^{3}\left(\mathbb{R}^{3}\right)$, which ensures continuity of all relevant traces across $\Gamma$ and also continuity of the mapping $\nabla \widetilde{\psi}_{\nu}: \mathrm{H}^{-1 / 2}(\Gamma) \rightarrow \mathbf{X}_{\mathrm{loc}}\left(\boldsymbol{\Delta}, \mathbb{R}^{3}\right)$. It seems that $\widetilde{\mathscr{G}}_{\nu}$ suffers a blow-up for $\nu \rightarrow 0$. However, note that, also owing to (4.2), there is an entire function $\tilde{g}_{6}: \mathbb{C}^{2} \rightarrow \mathbb{C}$ such that

$$
\nabla \widetilde{\mathscr{G}}_{\nu}(\boldsymbol{x})=\frac{1-\eta}{8 \pi \eta} \frac{\boldsymbol{x}}{|\boldsymbol{x}|}+\tilde{g}_{6}(\nu,|x|) \frac{\boldsymbol{x}}{|\boldsymbol{x}|} \in L^{\infty}\left(\mathbb{R}^{3}\right)^{3} .
$$

Hence, $\nabla \widetilde{\psi}_{\nu}$ turns out to be an entire function of $\nu \in \mathbb{C}$ ! 
5.6. Potentials for the Hodge-Helmholtz operator. Using the above notations, the Hodge-Helmholtz single layer potential (4.9) can be recast as

$$
\mathcal{S} \mathcal{L}_{\kappa}(\mathfrak{p})=-\Psi_{\kappa}(\mathbf{p})-\nabla \widetilde{\psi}_{\kappa}\left(\operatorname{div}_{\Gamma} \mathbf{p}\right)+\nabla \psi_{\tilde{\kappa}}(\alpha), \quad \mathfrak{p}=(\mathbf{p}, \alpha) \in \mathcal{H}_{\mathrm{N}}(\Gamma) .
$$

From the continuity properties previously established for $\nabla \psi_{\nu}, \widetilde{\psi}_{\nu}$ and $\Psi_{\nu}$, we deduce directly that $\mathcal{S} \mathcal{L}_{\kappa}$ continuously maps $\mathcal{H}_{\mathrm{N}}(\Gamma)$ into $\mathbf{X}_{\text {loc }}(\boldsymbol{\Delta}, \Omega)\left(\operatorname{resp} . \mathbf{X}_{\text {loc }}\left(\boldsymbol{\Delta}, \mathbb{R}^{3} \backslash \bar{\Omega}\right)\right)$. Moreover the jump identities (5.2)-(5.3) show that

$$
\left[\mathcal{T}_{\mathrm{D}}\right] \cdot \mathcal{S} \mathcal{L}_{\kappa}(\mathfrak{p})=0, \quad\left[\mathcal{T}_{\mathrm{N}}\right] \cdot \mathcal{S} \mathcal{L}_{\kappa}(\mathfrak{p})=\mathfrak{p} \quad \forall \mathfrak{p} \in \mathcal{H}_{\mathrm{N}}(\Gamma) .
$$

As regards the Hodge-Helmholtz double layer potential (4.11), we find for $\mathfrak{q}=(\mathbf{q}, \beta) \epsilon$ $\mathcal{H}_{\mathrm{D}}(\Gamma)$

$$
\mathcal{D} \mathcal{L}_{\kappa}(\mathfrak{q})=\operatorname{curl} \Psi_{\kappa}(\mathbf{q} \times \boldsymbol{n})+\Upsilon_{\nu}(\beta) .
$$

Thus, we can appeal to the jump formulas (5.4)-(5.5) to deduce

$$
\left[\mathcal{T}_{\mathrm{D}}\right] \cdot \mathcal{D} \mathcal{L}_{\kappa}(\mathfrak{p})=\mathfrak{p} \quad, \quad\left[\mathcal{T}_{\mathrm{N}}\right] \cdot \mathcal{D} \mathcal{L}_{\kappa}(\mathfrak{p})=0, \quad \forall \mathfrak{p}=(\mathbf{p}, \alpha) \in \mathcal{H}_{\mathrm{D}}(\Gamma) .
$$

The next theorem summarizes our findings.

THEOREM 5.1. We have the jump relations:

$$
\begin{array}{lll}
{\left[\mathcal{T}_{\mathrm{D}}\right] \cdot \mathcal{D} \mathcal{L}_{\kappa}(\mathfrak{p})=\mathfrak{p},} & {\left[\mathcal{T}_{\mathrm{N}}\right] \cdot \mathcal{D} \mathcal{L}_{\kappa}(\mathfrak{p})=0} & \forall \mathfrak{p} \in \mathcal{H}_{\mathrm{D}}(\Gamma), \\
{\left[\mathcal{T}_{\mathrm{D}}\right] \cdot \mathcal{S} \mathcal{L}_{\kappa}(\mathfrak{p})=0,} & {\left[\mathcal{T}_{\mathrm{N}}\right] \cdot \mathcal{S} \mathcal{L}_{\kappa}(\mathfrak{p})=\mathfrak{p}} & \forall \mathfrak{p} \in \mathcal{H}_{\mathrm{N}}(\Gamma)
\end{array}
$$

Note that, since both $\mathcal{T}_{\mathrm{D}}$ and $\mathcal{T}_{\mathrm{N}}$ are pairs of trace operators, the theorem above actually contains eight identities, not just four.

5.7. Calderón projector. So far, we have derived the representation formula (4.6) involving the layer potentials (4.9) and (4.11), and we established corresponding continuity properties. It is natural to define associated Calderón projectors, as this is a key concept in reformulating boundary value problems as integral equations [9, Thm. 8]. In the present context, the Calderón projector will be the continuous map

$$
\mathfrak{C}_{\kappa}:\left\{\begin{array}{cll}
\mathcal{H}_{\mathrm{D}}(\Gamma) \times \mathcal{H}_{\mathrm{N}}(\Gamma) & \rightarrow \mathcal{H}_{\mathrm{D}}(\Gamma) \times \mathcal{H}_{\mathrm{N}}(\Gamma) \\
{\left[\begin{array}{l}
\mathfrak{p} \\
\mathfrak{q}
\end{array}\right]} & \mapsto\left[\begin{array}{l}
\mathcal{T}_{\mathrm{D}} \cdot \mathcal{D} \mathcal{L}_{\kappa}(\mathfrak{p})+\mathcal{T}_{\mathrm{D}} \cdot \mathcal{S} \mathcal{L}_{\kappa}(\mathfrak{q}) \\
\mathcal{T}_{\mathrm{N}} \cdot \mathcal{D} \mathcal{L}_{\kappa}(\mathfrak{p})+\mathcal{T}_{\mathrm{N}} \cdot \mathcal{S} \mathcal{L}_{\kappa}(\mathfrak{q})
\end{array}\right]
\end{array}\right.
$$

Recall that the spaces $\mathcal{H}_{\mathrm{D}}(\Gamma), \mathcal{H}_{\mathrm{N}}(\Gamma)$ are products of two atomic trace spaces each, and that each operator $\mathcal{T}_{\mathrm{D}}, \mathcal{T}_{\mathrm{N}}$ involves a pair of trace operators. As a consequence $\mathfrak{C}_{\kappa}$ could be interpreted as a $4 \times 4$ matrix of boundary integral operators. It is a projector whose range caracterizes the traces of solutions to the equation $-\boldsymbol{\Delta}_{\eta} \mathbf{u}-\kappa^{2} \mathbf{u}=0$ in $\Omega$; we have a result analogous to [32, Prop. 3.6.2] or [9, Thm. 8].

Proposition 5.2. The operator $\mathfrak{C}_{\kappa}$ defined in (5.12) is a continuous projector. Its range (resp. kernel) are the traces of solutions of homogeneous inner (resp. outer) boundary value problem, in particular for $\kappa \neq 0$,

$$
\begin{array}{r}
\operatorname{range}\left(\mathfrak{C}_{\kappa}\right):=\left\{\left(\mathcal{T}_{\mathrm{D}}(\mathbf{u}), \mathcal{T}_{\mathrm{N}}(\mathbf{u})\right) \mid \mathbf{u} \in\right. \\
\quad \mathbf{X}_{\mathrm{loc}}(\boldsymbol{\Delta}, \bar{\Omega}), \boldsymbol{\Delta}_{\eta} \mathbf{u}+\kappa^{2} \mathbf{u}=0 \text { in } \Omega, \\
\text { satisfying (3.12)-(3.13), if } \Omega \text { is unbounded }\} \\
\operatorname{ker}\left(\mathfrak{C}_{\kappa}\right):=\left\{\left(\mathcal{T}_{\mathrm{D}, c}(\mathbf{u}), \mathcal{T}_{\mathrm{N}, c}(\mathbf{u})\right) \mid \mathbf{u} \in \mathbf{X}_{\mathrm{loc}}\left(\boldsymbol{\Delta}, \mathbb{R}^{3} \backslash \Omega\right), \boldsymbol{\Delta}_{\eta} \mathbf{u}+\kappa^{2} \mathbf{u}=0 \text { in } \mathbb{R}^{3} \backslash \bar{\Omega},\right. \\
\text { satisfying (3.12)-(3.13), if } \left.\mathbb{R}^{3} \backslash \bar{\Omega} \text { is unbounded }\right\}
\end{array}
$$


We do not provide the proof of this result because it follows from a classical argument (see e.g. [34, §6.6]). Essentially, the theorem is a direct consequence of the representation formula (4.6) and the jump relations of Theorem 5.1.

6. Boundary integral operators. This core section will be dedicated to the study of the invertibility of the first-kind boundary integral operators $\mathcal{T}_{\mathrm{D}} \cdot \mathcal{S} \mathcal{L}_{\kappa}$ and $\mathcal{T}_{\mathrm{N}} \cdot \mathcal{D} \mathcal{L}_{\kappa}$ associated with the Hodge-Helmholtz single and double layer potentials (4.9) and (4.11). Our notion of a first kind boundary integral operator is that of a mapping between (trace) spaces that are in duality with respect to an $L^{2}(\Gamma)$-type pairing. Then it is natural to adopt a variational perspective and study the induced bilinear forms on trace spaces.

Remark 6.1. We would like to point out two obstructions to applying the simple standard argument showing coercivity of first-kind boundary integral operators in the case of strongly elliptic PDEs [23, Thms. 7.6 \& 7.8]:

1. The observation (3.9) and the resulting failure of $\left\langle\mathfrak{p}, \mathcal{T}_{\mathrm{D}} \cdot \mathcal{S} \mathcal{L}_{0}(\mathfrak{p})\right\rangle$ to define an "energy trace norm" on $\mathcal{H}_{\mathrm{D}}(\Gamma)$.

2. The missing compact embedding of $\mathbf{X}(\Omega)$ into $L^{2}(\Omega)^{3}$ even for bounded $\Omega$.

6.1. Injectivity. Insights into the uniqueness of solutions of boundary value problems as gleaned in Proposition 3.7 and Corollary 3.9 permit us to determine, when the kernels of boundary integral operators will be trivial.

Proposition 6.2. Assume that $\Omega$ is a bounded Lipschitz domain.

(i) If $\kappa^{2} \in \mathbb{C}_{+} \backslash\left(\Lambda_{\mathrm{T}} \cup \eta \Lambda_{\mathrm{D}}\right)$ then the operators $\mathcal{T}_{\mathrm{D}} \cdot \mathcal{D} \mathcal{L}_{\kappa}: \mathcal{H}_{\mathrm{D}}(\Gamma) \rightarrow \mathcal{H}_{\mathrm{D}}(\Gamma)$ and $\mathcal{T}_{\mathrm{D}} \cdot \mathcal{S} \mathcal{L}_{\kappa}: \mathcal{H}_{\mathrm{N}}(\Gamma) \rightarrow \mathcal{H}_{\mathrm{D}}(\Gamma)$ are one-to-one.

(ii) If $\kappa^{2} \in \mathbb{C}_{+} \backslash\left(\Lambda_{\mathrm{R}} \cup \Lambda_{\mathrm{N}}\right)$ then the operators $\mathcal{T}_{\mathrm{N}} \cdot \mathcal{S} \mathcal{L}_{\kappa}: \mathcal{H}_{\mathrm{N}}(\Gamma) \rightarrow \mathcal{H}_{\mathrm{N}}(\Gamma)$ and $\mathcal{T}_{\mathrm{N}} \cdot \mathcal{D} \mathcal{L}_{\kappa}: \mathcal{H}_{\mathrm{D}}(\Gamma) \rightarrow \mathcal{H}_{\mathrm{N}}(\Gamma)$ are one-to-one.

Proof:

We show the result only for the first operator $\mathcal{T}_{\mathrm{D}} \cdot \mathcal{D} \mathcal{L}_{\kappa}$. The proof in all other cases is similar. Assume that $\kappa^{2} \notin \Lambda_{\mathrm{T}} \cup \eta \Lambda_{\mathrm{D}}$, and that $\mathcal{T}_{\mathrm{D}} \cdot \mathcal{D} \mathcal{L}_{\kappa}(\mathfrak{p})=0$ for some $\mathfrak{p} \in \mathcal{H}_{\mathrm{D}}(\Gamma)$.

Define $\mathbf{v}_{+}(\boldsymbol{x})=\mathcal{D} \mathcal{L}_{\kappa}(\mathfrak{p})(\boldsymbol{x})$ for $\boldsymbol{x} \in \Omega$. By construction $\mathbf{v}_{+} \in \mathbf{X}(\Omega)$ and it satisfies $-\boldsymbol{\Delta}_{\eta} \mathbf{v}_{+}-\kappa^{2} \mathbf{v}_{+}=0$ in $\Omega$ and $\mathcal{T}_{\mathrm{D}}\left(\mathbf{v}_{+}\right)=0$ on $\partial \Omega$. As a consequence $\mathbf{v}_{+}=0$ according to Proposition 3.7, which implies in particular $\mathcal{T}_{\mathrm{N}}\left(\mathbf{v}_{+}\right)=0$.

Now set $\mathbf{v}_{-}(\boldsymbol{x})=\mathcal{D} \mathcal{L}_{\kappa}(\mathfrak{p})(\boldsymbol{x})$ for $\boldsymbol{x} \in \mathbb{R}^{3} \backslash \bar{\Omega}$. By construction $\mathbf{v}_{-} \in \mathbf{X}_{\text {loc }}\left(\mathbb{R}^{3} \backslash \bar{\Omega}\right)$ and it satisfies $-\boldsymbol{\Delta}_{\eta} \mathbf{v}_{-}-\kappa^{2} \mathbf{v}_{-}=0$ in $\mathbb{R}^{3} \backslash \bar{\Omega}$ as well as the radiation conditions (3.12) and (3.13). It also satisfies $\mathcal{T}_{\mathrm{N}, c}\left(\mathbf{v}_{-}\right)=\mathcal{T}_{\mathrm{N}, c} \cdot \mathcal{D} \mathcal{L}_{\kappa}(\mathfrak{p})=\mathcal{T}_{\mathrm{N}}\left(\mathbf{v}_{+}\right)-\left[\mathcal{T}_{\mathrm{N}}\right] \cdot \mathcal{D} \mathcal{L}_{\kappa}(\mathfrak{p})=0$ according to Theorem 5.1. Applying Corollary 3.9, we conclude that $\mathbf{v}_{-}=0$ in $\mathbb{R}^{3} \backslash \bar{\Omega}$. Finally, this implies $\mathfrak{p}=\left[\mathcal{T}_{\mathrm{D}}\right] \cdot \mathcal{D} \mathcal{L}_{\kappa}(\mathfrak{p})=\mathcal{T}_{\mathrm{D}}\left(\mathbf{v}_{+}\right)-\mathcal{T}_{\mathrm{D}, c}\left(\mathbf{v}_{-}\right)=0$.

Similar arguments yield the analogous result in the case where $\mathbb{R}^{3} \backslash \bar{\Omega}$ is bounded (and not $\Omega$ ).

Proposition 6.3. Assume that $\mathbb{R}^{3} \backslash \bar{\Omega}$ is a bounded Lipschitz domain.

(i) If $\kappa^{2} \in \mathbb{C}_{+} \backslash\left(\Lambda_{\mathrm{T}} \cup \eta \Lambda_{\mathrm{D}}\right)$, then the operators $\mathcal{T}_{\mathrm{N}} \cdot \mathcal{S L}_{\kappa}: \mathcal{H}_{\mathrm{N}}(\Gamma) \rightarrow \mathcal{H}_{\mathrm{N}}(\Gamma)$ and $\mathcal{T}_{\mathrm{D}} \cdot \mathcal{S} \mathcal{L}_{\kappa}: \mathcal{H}_{\mathrm{N}}(\Gamma) \rightarrow \mathcal{H}_{\mathrm{D}}(\Gamma)$ are one-to-one.

(ii) If $\kappa^{2} \in \mathbb{C}_{+} \backslash\left(\Lambda_{\mathrm{R}} \cup \eta \Lambda_{\mathrm{N}}\right)$, then the operators $\mathcal{T}_{\mathrm{D}} \cdot \mathcal{D} \mathcal{L}_{\kappa}: \mathcal{H}_{\mathrm{D}}(\Gamma) \rightarrow \mathcal{H}_{\mathrm{D}}(\Gamma)$ and $\mathcal{T}_{\mathrm{N}} \cdot \mathcal{D} \mathcal{L}_{\kappa}: \mathcal{H}_{\mathrm{D}}(\Gamma) \rightarrow \mathcal{H}_{\mathrm{N}}(\Gamma)$ are one-to-one. 
6.2. Tools. To simplify notations we introduce the inner products

$$
\begin{aligned}
& (\alpha, \beta)_{-1 / 2}:=\int_{\Gamma \times \Gamma} \mathscr{G}_{0}(\boldsymbol{x}-\boldsymbol{y}) \alpha(\boldsymbol{x}) \overline{\beta(\boldsymbol{y})} d \sigma(\boldsymbol{x}, \boldsymbol{y}), \quad \alpha, \beta \in \mathrm{H}^{-1 / 2}(\Gamma), \\
& (\mathbf{p}, \mathbf{q})_{-1 / 2}:=\int_{\Gamma \times \Gamma} \mathscr{G}_{0}(\boldsymbol{x}-\boldsymbol{y}) \mathbf{p}(\boldsymbol{x}) \overline{\mathbf{q}(\boldsymbol{y})} d \sigma(\boldsymbol{x}, \boldsymbol{y}), \quad \mathbf{p}, \mathbf{q} \in \mathbf{H}_{\mathrm{R}}^{-1 / 2}(\Gamma) / \mathbf{H}_{\mathrm{T}}^{-1 / 2}(\Gamma) .
\end{aligned}
$$

Both sesqilinear forms are strongly coercive in $\mathrm{H}^{-1 / 2}(\Gamma) / \mathbf{H}_{\mathrm{R}}^{-1 / 2}(\Gamma) / \mathbf{H}_{\mathrm{T}}^{-1 / 2}(\Gamma)$, and, thus, they induce equivalent norms $\|\cdot\|_{-1 / 2}$ on these spaces. This is a classical result of potential theory, see e.g. [32, Thm. 3.5.4]. Thus, in all estimates, we can replace $\|\cdot\|_{\mathrm{H}^{-1 / 2}(\Gamma)},\|\cdot\|_{\mathbf{H}_{\mathrm{T}}^{-1 / 2}(\Gamma)}$, and $\|\cdot\|_{\mathbf{H}_{\mathrm{r}}^{-1 / 2}(\Gamma)}$ with $\|\cdot\|_{-1 / 2}$, and we will do so.

In the study of first-kind boundary integral operators mapping properties of the surface divergence $\operatorname{div}_{\Gamma}$ will be crucial and are summarized in the next lemma. For the sake of brevity we write

$$
\mathrm{H}_{*}^{ \pm 1 / 2}(\Gamma):=\left\{\begin{array}{l}
\varphi \in \mathrm{H}^{ \pm 1 / 2}(\Gamma) \mid\left\langle\varphi, \mathbf{1}_{C}\right\rangle=0 \\
\text { for all connected components } C \text { of } \Gamma
\end{array}\right\} .
$$

Here $\mathbf{1}_{C}$ denotes the characteristic function of a subset $C \subset \Gamma$. This spawns straightforward "orthogonal" direct decompositions

$$
\mathrm{H}^{ \pm 1 / 2}(\Gamma)=\mathrm{H}_{*}^{ \pm 1 / 2}(\Gamma) \oplus \mathrm{C}_{*}(\Gamma)
$$

where $\mathrm{C}_{*}(\Gamma)$ is the finite-dimensional space spanned by the characteristic functions of the connected components of $\Gamma$. For later use we introduce the projectors $Q_{*}$ : $\mathrm{H}^{ \pm 1 / 2}(\Gamma) \rightarrow \mathrm{H}_{*}^{ \pm 1 / 2}(\Gamma)$ and $\mathrm{Q}_{c}: \mathrm{H}^{ \pm 1 / 2}(\Gamma) \rightarrow \mathrm{C}_{*}(\Gamma)$ induced by $(6.1)$. Which version to take will be clear from the context.

LEMmA 6.4. The surface divergence $\operatorname{div}_{\Gamma}$ is a bounded surjective operator

$$
\operatorname{div}_{\Gamma}: \mathbf{H}^{-1 / 2}(\operatorname{div}, \Gamma) \rightarrow \mathrm{H}_{\star}^{-1 / 2}(\Gamma) .
$$

The surface rotation $\operatorname{curl}_{\Gamma}:=\nabla_{\Gamma} \times \boldsymbol{n}$ is a bounded injective operator

$$
\operatorname{curl}_{\Gamma}: \mathrm{H}_{*}^{1 / 2}(\Gamma) \rightarrow \mathbf{H}^{-1 / 2}(\operatorname{div}, \Gamma),
$$

with closed range and satisfies $\operatorname{div}_{\Gamma} \circ \operatorname{curl}_{\Gamma}=0$.

As another tool we will rely on Hodge-type decompositions of $\operatorname{div}_{\Gamma}$-conforming tangential surface vector fields, established, for example, in [9, Lemma 2].

Lemma 6.5. There exists a continuous projector $\mathrm{R}_{\Gamma}: \mathbf{H}^{-1 / 2}(\operatorname{div}, \Gamma) \rightarrow \mathbf{H}^{-1 / 2}(\operatorname{div}, \Gamma)$ that

(i) is compact as a mapping $\mathbf{H}^{-1 / 2}(\operatorname{div}, \Gamma) \rightarrow \mathbf{H}_{\mathrm{R}}^{-1 / 2}(\Gamma)$,

(ii) leaves the surface divergence of its argument invariant: $\operatorname{div}_{\Gamma} \circ \mathrm{R}_{\Gamma}=\operatorname{div}_{\Gamma}$,

(iii) and satisfies $\operatorname{ker}\left(\mathrm{R}_{\Gamma}\right)=\mathbf{H}^{-1 / 2}(\operatorname{div} 0, \Gamma):=\left\{\mathbf{p} \in \mathbf{H}^{-1 / 2}(\operatorname{div}, \Gamma), \operatorname{div}_{\Gamma} \mathbf{p}=0\right\}$.

To begin with, the projector $\mathrm{R}_{\Gamma}$ induces a stable direct decomposition of $\mathbf{H}^{-1 / 2}(\operatorname{div}, \Gamma)$ into closed subspaces:

$$
\mathbf{H}^{-1 / 2}(\operatorname{div}, \Gamma)=\mathbf{H}^{-1 / 2}(\operatorname{div} 0, \Gamma) \oplus \mathbf{Y}_{\perp}, \quad \mathbf{Y}_{\perp}=\operatorname{ker}\left(\operatorname{Id}-\mathrm{R}_{\Gamma}\right)=\mathrm{R}_{\Gamma}\left(\mathbf{H}^{-1 / 2}(\operatorname{div}, \Gamma)\right) .
$$

Stability of the decomposition together with (iii) translates into an equivalence of norms

$$
\|\mathbf{p}\|_{\mathbf{H}^{-1 / 2}(\operatorname{div}, \Gamma)} \approx\left\|\operatorname{div}_{\Gamma} \mathbf{p}\right\|_{-1 / 2}+\left\|\left(\operatorname{Id}-\mathrm{R}_{\Gamma}\right) \mathbf{p}\right\|_{-1 / 2} \quad \forall \mathbf{p} \in \mathbf{H}^{-1 / 2}\left(\operatorname{div}_{\Gamma}, \Gamma\right) .
$$


Combining Lemmas 6.4, 6.5, and appealing to the open mapping theorem yields the existence of bounded one-sided inverses of the surface differential operators

$$
\begin{array}{ll}
\operatorname{div}_{\Gamma}^{\dagger}: \mathrm{H}_{*}^{-1 / 2}(\Gamma) \rightarrow \mathbf{Y}_{\perp}, & \operatorname{div}_{\Gamma} \circ \operatorname{div}_{\Gamma}^{\dagger}=\mathrm{Id}, \\
\operatorname{curl}_{\Gamma}^{\dagger}: \mathbf{H}^{-1 / 2}(\operatorname{div} 0, \Gamma) \rightarrow \mathrm{H}_{*}^{1 / 2}(\Gamma), & \operatorname{curl}_{\Gamma}^{\dagger} \circ \operatorname{curl}_{\Gamma}=\mathrm{Id} .
\end{array}
$$

Moreover, $\operatorname{curl}_{\Gamma}^{\dagger}$ can be chosen such that

$$
\operatorname{curl}_{\Gamma} \circ \operatorname{curl}_{\Gamma}^{\dagger}=\mathrm{P}_{0} \quad \text { on } \mathbf{H}^{-1 / 2}(\operatorname{div} 0, \Gamma),
$$

where $\mathrm{P}_{0}: \mathbf{H}^{-1 / 2}(\operatorname{div} 0, \Gamma) \rightarrow \operatorname{curl}_{\Gamma} \mathrm{H}_{*}^{1 / 2}(\Gamma) \subset \mathbf{H}^{-1 / 2}(\operatorname{div} 0, \Gamma)$ is the $\mathbf{H}^{-1 / 2}(\operatorname{div}, \Gamma)$ orthogonal projection.

6.3. Boundary integral operator $\mathcal{T}_{\mathrm{D}} \cdot \mathcal{S} \mathcal{L}_{\kappa}$ : Coercivity. We shall now take a closer look at the operator $\mathcal{T}_{\mathrm{D}} \cdot \mathcal{S} \mathcal{L}_{\kappa}$. We first start by deriving convenient expressions for the bilinear form associated to it, reusing the potential operators $\psi_{\kappa}, \Psi_{\kappa}$ introduced in Section 5. Recall (5.8):

$\mathcal{S} \mathcal{L}_{\kappa}(\mathfrak{u})=-\Psi_{\kappa}(\mathbf{p})-\nabla \widetilde{\psi}_{\kappa}\left(\operatorname{div}_{\Gamma} \mathbf{p}\right)+\nabla \psi_{\tilde{\kappa}}(\alpha) \quad \forall \mathfrak{u}=(\mathbf{p}, \alpha) \in \mathcal{H}_{\mathrm{N}}(\Gamma)=\mathbf{H}^{-\frac{1}{2}}(\operatorname{div}, \Gamma) \times \mathrm{H}^{-\frac{1}{2}}(\Gamma)$.

Next, we use the surface Green's formula $\int_{\Gamma} \mathbf{q} \cdot \gamma_{\mathrm{T}}(\nabla U) d \sigma=-\int_{\Gamma} \operatorname{div}_{\Gamma}(\mathbf{q}) \cdot \tau_{\mathrm{D}}(U) d \sigma$ for any $U \in \mathrm{H}_{\mathrm{loc}}^{1}(\bar{\Omega})$ and any $\mathbf{q} \in \mathbf{H}^{-1 / 2}(\operatorname{div}, \Gamma)$. Applying this formula to the above potential yields the following expression $\left(\nabla \widetilde{\mathscr{G}}_{\kappa}\right.$ is given in $(5.7)$ and vanishes for $\left.\eta=1\right)$

$$
\begin{aligned}
\left\langle\mathbf{q}, \gamma_{\mathrm{T}} \cdot \mathcal{S} \mathcal{L}_{\kappa}(\mathfrak{u})\right\rangle= & -\int_{\Gamma \times \Gamma} \mathscr{G}_{\kappa}(\boldsymbol{x}-\boldsymbol{y}) \mathbf{p}(\boldsymbol{y}) \cdot \mathbf{q}(\boldsymbol{x}) d \sigma(\boldsymbol{x}, \boldsymbol{y}) \\
& -\int_{\Gamma \times \Gamma}\left(\nabla \widetilde{\mathscr{G}_{\kappa}}\right)(\boldsymbol{x}-\boldsymbol{y}) \operatorname{div}_{\Gamma} \mathbf{p}(\boldsymbol{y}) \cdot \mathbf{q}(\boldsymbol{x}) d \sigma(\boldsymbol{x}, \boldsymbol{y}) \\
& -\int_{\Gamma \times \Gamma} \mathscr{G}_{\tilde{\kappa}}(\boldsymbol{x}-\boldsymbol{y}) \alpha(\boldsymbol{y}) \cdot \operatorname{div}_{\Gamma} \mathbf{q}(\boldsymbol{x}) d \sigma(\boldsymbol{x}, \boldsymbol{y}),
\end{aligned}
$$

where, for the sake of conciseness, we have written $d \sigma(\boldsymbol{x}, \boldsymbol{y})$ for the product surface measure on $\Gamma \times \Gamma$. Since $\mathscr{G}_{\kappa}(\boldsymbol{x})$ solves the Helmholtz equation, we find $\Delta \psi_{\kappa}(\beta)=$ $-\kappa^{2} \psi_{\kappa}(\beta)$ and $\Delta \widetilde{\psi}_{\kappa}(\beta)=-\psi_{\kappa}(\beta)+\eta^{-1} \psi_{\tilde{\kappa}}(\beta)$ for any $\beta \in \mathrm{H}^{-1 / 2}(\Gamma)$, since $\tilde{\kappa}^{2}=\eta^{-1} \kappa^{2}$. Besides we have $\operatorname{div}\left(\Psi_{\kappa}(\mathbf{p})\right)=\psi_{\kappa}\left(\operatorname{div}_{\Gamma} \mathbf{p}\right)$ according to [22, Lemma 2.3]. As a consequence

$$
\begin{aligned}
\eta \operatorname{div}\left(\mathcal{S} \mathcal{L}_{\kappa}(\mathfrak{u})\right) & =\eta \psi_{\kappa}\left(\operatorname{div}_{\Gamma} \mathbf{p}\right)-\psi_{\tilde{\kappa}}\left(\operatorname{div}_{\Gamma} \mathbf{p}\right)-\eta \psi_{\kappa}\left(\operatorname{div}_{\Gamma} \mathbf{p}\right)-\kappa^{2} \psi_{\tilde{\kappa}}(\alpha) \\
& =-\psi_{\tilde{\kappa}}\left(\operatorname{div}_{\Gamma} \mathbf{p}\right)-\kappa^{2} \psi_{\tilde{\kappa}}(\alpha)
\end{aligned}
$$

Now recall that $\gamma_{\mathrm{D}}(u):=\left.\operatorname{div}(u)\right|_{\Gamma}$. Thus, (6.7) and (6.8) show that, for any $\mathfrak{u}=(\mathbf{p}, \alpha) \epsilon$ $\mathcal{H}_{\mathrm{N}}(\Gamma)$ and any $\mathfrak{v}=(\mathbf{q}, \beta) \in \mathcal{H}_{\mathrm{N}}(\Gamma)$, we have

$$
\begin{aligned}
\left\langle\mathcal{T}_{\mathrm{D}} \cdot \mathcal{S L}_{\kappa}(\mathfrak{u}), \mathfrak{v}\right\rangle= \\
\quad-\int_{\Gamma \times \Gamma} \mathscr{G}_{\tilde{\kappa}}(\boldsymbol{x}-\boldsymbol{y})\left[\mathbf{p}(\boldsymbol{y}) \cdot \mathbf{q}(\boldsymbol{x})+\alpha(\boldsymbol{y}) \cdot \operatorname{div}_{\Gamma} \mathbf{q}(\boldsymbol{x})\right] d \sigma(\boldsymbol{x}, \boldsymbol{y}) \\
\quad-\int_{\Gamma \times \Gamma} \mathscr{G}_{\tilde{\kappa}}(\boldsymbol{x}-\boldsymbol{y})\left[\operatorname{div}_{\Gamma} \mathbf{p}(\boldsymbol{y}) \beta(\boldsymbol{x})+\kappa^{2} \alpha(\boldsymbol{y}) \beta(\boldsymbol{x})\right] d \sigma(\boldsymbol{x}, \boldsymbol{y}) \\
\quad-\int_{\Gamma \times \Gamma} \widetilde{\mathscr{G}}_{\kappa}(\boldsymbol{x}-\boldsymbol{y})\left[\operatorname{div}_{\Gamma} \mathbf{q}(\boldsymbol{x}) \operatorname{div}_{\Gamma} \mathbf{p}(\boldsymbol{y})-\kappa^{2} \mathbf{p}(\boldsymbol{x}) \cdot \mathbf{q}(\boldsymbol{y})\right] d \sigma(\boldsymbol{x}, \boldsymbol{y})
\end{aligned}
$$

We point out that all the integrals depend analytically on $\kappa \in \mathbb{C}$. 
Remark 6.6. We hasten to add that, strictly speaking, all integrals have to be read as duality pairings. For $\mathbf{p}, \mathbf{q}, \alpha, \beta \in \mathrm{L}^{\infty}(\Gamma)$ an interpretation as classical improper integrals is possible.

Some terms of the above bilinear form are just compact contributions that do not require a detailed analysis.

Proposition 6.7. Define $\mathrm{A}_{\mathrm{SL}}: \mathcal{H}_{\mathrm{N}}(\Gamma) \rightarrow \mathcal{H}_{\mathrm{D}}(\Gamma)$ through

$$
\begin{aligned}
\left\langle\mathrm{A}_{\mathrm{SL}}(\mathfrak{u}), \mathfrak{v}\right\rangle:= & \int_{\Gamma \times \Gamma} \mathscr{G}_{0}(\boldsymbol{x}-\boldsymbol{y})\left[\mathbf{p}(\boldsymbol{y}) \cdot \mathbf{q}(\boldsymbol{x})+\alpha(\boldsymbol{y}) \operatorname{div}_{\Gamma} \mathbf{q}(\boldsymbol{x})\right] d \sigma(\boldsymbol{x}, \boldsymbol{y})+ \\
& \int_{\Gamma \times \Gamma} \mathscr{G}_{0}(\boldsymbol{x}-\boldsymbol{y})\left[\operatorname{div}_{\Gamma} \mathbf{p}(\boldsymbol{y}) \beta(\boldsymbol{x})+\kappa^{2} \alpha(\boldsymbol{y}) \beta(\boldsymbol{x})\right] d \sigma(\boldsymbol{x}, \boldsymbol{y}),
\end{aligned}
$$

for $\mathfrak{u}=(\mathbf{p}, \alpha), \mathfrak{v}=(\mathbf{q}, \beta) \in \mathcal{H}_{\mathrm{N}}(\Gamma)$. Then the operator $\mathcal{T}_{\mathrm{D}} \cdot \mathcal{S} \mathcal{L}_{\kappa}+\mathrm{A}_{\mathrm{SL}}$ is an analytic function of $\kappa \in \mathbb{C}$ into the space of compact operators $\mathcal{H}_{\mathrm{N}}(\Gamma) \mapsto \mathcal{H}_{\mathrm{D}}(\Gamma)$.

Proof:

First of all note that the third term in (6.9) is compact, because (5.7) shows that the kernel $\nabla \widetilde{\mathscr{G}}_{\kappa}$ is a pseudo-homogeneous kernel of degree 0 . The arguments of Section 5.5 plus the Rellich embedding theorem yield compactness.

Secondly, reasoning as in the proof of [32, Lemma 3.9.8] we see that replacing $\mathscr{G}_{\tilde{\kappa}}$ by $\mathscr{G}_{0}$ in the first and second term of (6.9) induces only a compact perturbation. This concludes the proof.

Now we are going to study the coercivity of the bilinear form (6.9). Thanks to Proposition 6.7, we can focus on $\mathrm{A}_{\mathrm{SL}}$ only. For the selection of "candidate functions" in coercivity estimates we introduce continuous operators $\Xi_{\kappa}: \mathcal{H}_{\mathrm{N}}(\Gamma) \rightarrow \mathcal{H}_{\mathrm{N}}(\Gamma)$ defined by the formula (projectors $\mathrm{Q}_{*}$ and $\mathrm{Q}_{c}$ induced by (6.1))

$$
\Xi_{\kappa}(\mathbf{p}, \alpha):=\left(\left(\operatorname{Id}-\mathrm{R}_{\Gamma}\right) \mathbf{p}+\nu \operatorname{div}_{\Gamma}^{\dagger} \mathrm{Q}_{*} \alpha, \operatorname{div}_{\Gamma} \mathbf{p}+\mathrm{Q}_{c} \alpha\right), \quad \nu:=1+\frac{1}{2}|\kappa|^{4} .
$$

Lemma 6.5 furnishes the relationships

$$
\operatorname{div}_{\Gamma} \circ\left(\operatorname{Id}-\mathrm{R}_{\Gamma}\right)=0, \quad\left(\operatorname{Id}-\mathrm{R}_{\Gamma}\right) \circ \operatorname{div}_{\Gamma}^{\dagger}=0,
$$

which, together with the projector properties of $\mathrm{Q}_{*}$, can be used to show that the bounded operators mapping $\mathbf{H}^{-1 / 2}(\operatorname{div}, \Gamma) \rightarrow \mathbf{H}^{-1 / 2}(\operatorname{div}, \Gamma)$, and defined as

$$
\left.(\mathbf{q}, \beta) \mapsto\left(\left(\mathrm{Id}-\mathrm{R}_{\Gamma}\right) \mathbf{q}+\operatorname{div}_{\Gamma}^{\dagger} \mathrm{Q}_{*} \beta, \nu^{-1} \operatorname{div}_{\Gamma} \mathbf{q}+\mathrm{Q}_{c} \beta\right),\right)
$$

are inverses of $\Xi_{\kappa}$ for every $\kappa \in \mathbb{C}$. Therefore, $\Xi_{\kappa}$ is an automorphism of $\mathbf{H}^{-1 / 2}(\operatorname{div}, \Gamma)$ for every $\kappa \in \mathbb{C}_{+}$, continuously depending on $\kappa$.

TheOREM 6.8. For any $\kappa \in \mathbb{C}$ there exists a compact operator $\mathrm{K}_{\kappa}: \mathcal{H}_{\mathrm{N}}(\Gamma) \rightarrow$ $\mathcal{H}_{\mathrm{D}}(\Gamma)$, and a constant $C=C(\kappa)>0$, such that

$$
\left.\mathfrak{R} e\left\{\left\langle\left(\mathcal{T}_{\mathrm{D}} \cdot \mathcal{S} \mathcal{L}_{\kappa}+\mathrm{K}_{\kappa}\right) \mathfrak{u}, \Xi(\overline{\mathfrak{u}})\right\rangle\right\}\right\} \geq C(\kappa)\|\mathfrak{u}\|_{\mathcal{H}_{N}(\Gamma)}^{2} \quad \forall \mathfrak{u} \in \mathcal{H}_{\mathrm{N}}(\Gamma) .
$$

Both, $C(\kappa)$ and $\mathrm{K}_{\kappa}$ depend continuously on $\kappa$.

\section{Proof:}

Since $\mathcal{T}_{\mathrm{D}} \cdot \mathcal{S} \mathcal{L}_{\kappa}$ only differs from $\mathrm{A}_{\mathrm{SL}}$ by a compact perturbation according to Proposition 6.7 , it suffices to prove $(6.13)$ with $\mathcal{T}_{\mathrm{D}} \cdot \mathcal{S} \mathcal{L}_{\kappa}$ replaced by $\mathrm{A}_{\mathrm{SL}}$. Using the notation $(\cdot, \cdot)_{-1 / 2}$ from Section 6.2 we can write, for $\mathfrak{u}=(\mathbf{p}, \alpha), \mathfrak{v}=(\mathbf{q}, \beta) \in \mathcal{H}_{\mathrm{N}}(\Gamma)$,

$$
\left\langle\left\langle\mathrm{A}_{\mathrm{SL}}(\mathfrak{u}), \overline{\mathfrak{v}}\right\rangle=(\mathbf{p}, \mathbf{q})_{-1 / 2}+\left(\alpha, \operatorname{div}_{\Gamma} \mathbf{q}\right)_{-1 / 2}+\left(\operatorname{div}_{\Gamma} \mathbf{p}, \beta\right)_{-1 / 2}+\kappa^{2}(\alpha, \beta)_{-1 / 2} .\right.
$$


Plugging (6.11) into the expression (6.14) yields

$$
\begin{aligned}
\left\langle\mathrm{A}_{\mathrm{SL}}(\mathfrak{u}), \Xi(\overline{\mathfrak{u}})\right\rangle= & \left(\left(\mathrm{Id}-\mathrm{R}_{\Gamma}\right) \mathbf{p}+\mathrm{R}_{\Gamma} \mathbf{p},\left(\mathrm{Id}-\mathrm{R}_{\Gamma}\right) \mathbf{p}+\nu \operatorname{div}_{\Gamma}^{\dagger} \mathrm{Q}_{*} \beta\right)_{-1 / 2}+ \\
& (\mathrm{Q}_{*} \alpha+\mathrm{Q}_{c} \alpha, \underbrace{\operatorname{div}_{\Gamma}\left(\mathrm{Id}-\mathrm{R}_{\Gamma}\right) \mathbf{p}}_{=0}+\nu \underbrace{\operatorname{div}_{\Gamma} \operatorname{div}_{\Gamma}^{\dagger}}_{=\operatorname{Id}_{\Gamma}} \mathrm{Q}_{*} \alpha)_{-1 / 2}+ \\
& \left(\operatorname{div}_{\Gamma} \mathbf{p}, \operatorname{div}_{\Gamma} \mathbf{p}+\mathrm{Q}_{c} \alpha\right)_{-1 / 2}+\kappa^{2}\left(\alpha, \operatorname{div}_{\Gamma} \mathbf{p}+\mathrm{Q}_{c} \alpha\right)_{-1 / 2} \\
= & \mathrm{B}_{\kappa}(\mathfrak{u}, \mathfrak{u})+\mathrm{C}_{\kappa}(\mathfrak{u}, \mathfrak{u}),
\end{aligned}
$$

with expressions

$$
\begin{aligned}
\mathrm{B}_{\kappa}(\mathfrak{u}, \mathfrak{u}):= & \left\|\left(\mathrm{Id}-\mathrm{R}_{\Gamma}\right) \mathbf{p}\right\|_{-1 / 2}^{2}+\nu\|\alpha\|_{-1 / 2}^{2}+\left\|\operatorname{div}_{\Gamma} \mathbf{p}\right\|_{-1 / 2}^{2}+\kappa^{2}\left(\alpha, \operatorname{div}_{\Gamma} \mathbf{p}\right)_{-1 / 2}, \\
\mathrm{C}_{\kappa}(\mathfrak{u}, \mathfrak{u}):= & \left(\mathrm{R}_{\Gamma} \mathbf{p},\left(\mathrm{Id}-\mathrm{R}_{\Gamma}\right) \mathbf{p}\right)_{-1 / 2}+\left(\left(\operatorname{Id}-\mathrm{R}_{\Gamma}\right) \mathbf{p}, \nu \operatorname{div}_{\Gamma}^{\dagger} \mathrm{Q}_{*} \alpha\right)_{-1 / 2}+\left(\mathrm{R}_{\Gamma} \mathbf{p}, \nu \operatorname{div}_{\Gamma}^{\dagger} \mathrm{Q}_{*} \alpha\right)_{-1 / 2}+ \\
& \left(\operatorname{div}_{\Gamma} \mathbf{p}, \mathrm{Q}_{c} \alpha\right)_{-1 / 2}+\left(\kappa^{2}-\nu\right)\left(\alpha, \mathrm{Q}_{c} \alpha\right)_{-1 / 2}
\end{aligned}
$$

that can obviously be derived from sesqui-linear forms.

From Lemma 6.5 (i) and the fact that $\mathrm{Q}_{c}$ has finite rank, we learn that the second sesqui-linear form $\mathrm{C}_{\kappa}(\cdot, \cdot)$ induces a compact operator, which depends polynomially on $\kappa \in \mathbb{C}$. So we are left with proving that the first bilinear form $\mathrm{B}(\cdot, \cdot)$ is strongly coercive on $\mathcal{H}_{\mathrm{N}}(\Gamma)$. To begin with, Young's inequality permits us to estimate

$$
\begin{aligned}
\left|\mathrm{B}_{\kappa}(\mathfrak{u}, \mathfrak{u})\right| \geq & \left\|\left(\operatorname{Id}-\mathrm{R}_{\Gamma}\right) \mathbf{p}\right\|_{-1 / 2}^{2}+\nu\|\alpha\|_{-1 / 2}^{2}+\left\|\operatorname{div}_{\Gamma} \mathbf{p}\right\|_{-1 / 2}^{2} \\
& -\frac{1}{2}|\kappa|^{2}\left(\epsilon\|\alpha\|_{-1 / 2}^{2}+\frac{1}{\epsilon}\left\|\operatorname{div}_{\Gamma} \mathbf{p}\right\|_{-1 / 2}^{2}\right) \\
\geq & \left\|\left(\operatorname{Id}-\mathrm{R}_{\Gamma}\right) \mathbf{p}\right\|_{-1 / 2}^{2}+\left(\nu-\frac{1}{2}|\kappa|^{2} \epsilon\right)\|\alpha\|_{-1 / 2}^{2}+\left(1-\frac{1}{2 \epsilon}|\kappa|^{2}\right)\left\|\operatorname{div}_{\Gamma} \mathbf{p}\right\|_{-1 / 2}^{2},
\end{aligned}
$$

for any $\epsilon>0$. Plugging (6.3) into this estimate along with setting $\epsilon:=\kappa^{2}$ and $\nu:=1+|\kappa|^{4}$ yields

$$
|\mathrm{B}(\mathfrak{u}, \mathfrak{u})| \geq\left\|\left(\operatorname{Id}-\mathrm{R}_{\Gamma}\right) \mathbf{p}\right\|_{-1 / 2}^{2}+\left\|\mathrm{Q}_{*} \alpha\right\|_{-1 / 2}^{2}+\frac{1}{2}\left\|\operatorname{div}_{\Gamma} \mathbf{p}\right\|_{-1 / 2}^{2} .
$$

Appealing to (6.3) finishes the proof.

\section{Corollary 6.9 .}

(i) The operator $\mathcal{T}_{\mathrm{D}} \cdot \mathcal{S} \mathcal{L}_{\kappa}: \mathcal{H}_{\mathrm{N}}(\Gamma) \rightarrow \mathcal{H}_{\mathrm{D}}(\Gamma)$ is Fredholm with index 0 .

(ii) Moreover, if $\kappa^{2} \in \mathbb{C}_{+} \backslash\left(\Lambda_{\mathrm{T}} \cup \eta \Lambda_{\mathrm{D}}\right)$ then $\mathcal{T}_{\mathrm{D}} \cdot \mathcal{S} \mathcal{L}_{\kappa}$ is an isomorphism.

Proof:

Since $\Xi_{\kappa}$ is bijective, Theorem 6.8 implies that $\mathcal{T}_{\mathrm{D}} \cdot \mathcal{S} \mathcal{L}_{\kappa}+\mathrm{K}_{\kappa}$ is an isomorphism, where $\mathrm{K}_{\kappa}: \mathcal{H}_{\mathrm{N}}(\Gamma) \rightarrow \mathcal{H}_{\mathrm{D}}(\Gamma)$ is the compact operator coming into play in (6.13). Hence $\mathcal{T}_{\mathrm{D}} \cdot \mathcal{S} \mathcal{L}_{\kappa}$ is a compact perturbation of an isomoprhism, so it is of Fredholm type with index 0 according to classical Riesz-Fredholm theory, see e.g. [23, Thm. 2.26]. Moreover, in the case $\kappa^{2} \notin \Lambda_{\mathrm{T}} \cup \eta \Lambda_{\mathrm{D}}$, the operator $\mathcal{T}_{\mathrm{D}} \cdot \mathcal{S} \mathcal{L}_{\kappa}$ is one-to-one according to Proposition 6.2 and 6.3, which implies that it is an isomorphism in this case.

6.4. Boundary integral operator $\mathcal{T}_{\mathrm{N}} \cdot \mathcal{D} \mathcal{L}_{\kappa}$ : Coercivity. We now focus on the operator $\mathcal{T}_{\mathrm{N}} \cdot \mathcal{D} \mathcal{L}_{\kappa}$ that in our theory plays a role similar to that of the hypersingular operator in classical potential theory for the Helmholtz equation, see e.g. [32, §3.3.4]. Using the notations introduced in Section 5 above, we have from (4.11) for all $\mathfrak{u}=$ $(\mathbf{p}, \alpha) \in \mathcal{H}_{\mathrm{D}}(\Gamma)=\mathbf{H}^{-\frac{1}{2}}(\operatorname{curl}, \Gamma) \times \mathrm{H}^{+\frac{1}{2}}(\Gamma)$

$$
\mathcal{D} \mathcal{L}_{\kappa}(\mathfrak{u})=\operatorname{curl} \Psi_{\kappa}(\mathbf{p} \times \boldsymbol{n})+\Upsilon_{\kappa}(\alpha)
$$


As a first step, we derive the bilinear form induced by the operator $\mathcal{T}_{\mathrm{N}} \cdot \mathcal{D} \mathcal{L}_{\kappa}$. First of all observe that $\operatorname{curl}_{\boldsymbol{x}}(\mathbb{G}(\boldsymbol{x}-\boldsymbol{y}) \cdot \mathbf{a}(\boldsymbol{y}))=\operatorname{curl}_{\boldsymbol{x}}\left(\mathscr{G}_{\kappa}(\boldsymbol{x}-\boldsymbol{y}) \mathbf{a}(\boldsymbol{y})\right)=\left(\nabla \mathscr{G}_{\kappa}\right)(\boldsymbol{x}-\boldsymbol{y}) \times \mathbf{a}(\boldsymbol{y})$ for any $\mathbf{a} \in C^{0}(\Gamma)^{3}$, where $\mathbf{c u r l}_{\boldsymbol{x}}$ refers to the standard curl operator acting on $\mathbb{G}(\boldsymbol{x}-\boldsymbol{y})$ as a function of $\boldsymbol{x}$. From this we conclude that $\operatorname{curl} \Upsilon_{\kappa}(\alpha)(\boldsymbol{x})=\int_{\Gamma}\left(\nabla \mathscr{G}_{\kappa}\right)(\boldsymbol{x}-\boldsymbol{y}) \times$ $\boldsymbol{n}(\boldsymbol{y}) \alpha(\boldsymbol{y}) d \sigma(\boldsymbol{y})$. To further transform this expression, we use the vector calculus formula

$$
\int_{\Gamma} u(\boldsymbol{x}) \mathbf{a} \cdot\left(\boldsymbol{n}(\boldsymbol{x}) \times \nabla_{\Gamma} v(\boldsymbol{x})\right) d \sigma(\boldsymbol{x})=-\int_{\Gamma} v(\boldsymbol{x}) \mathbf{a} \cdot\left(\boldsymbol{n}(\boldsymbol{x}) \times \nabla_{\Gamma} u(\boldsymbol{x})\right) d \sigma(\boldsymbol{x})
$$

for all $\mathbf{a} \in \mathbb{R}^{3}, u, v \in \mathrm{H}^{1 / 2}(\Gamma)$. We can apply this formula taking $u(\boldsymbol{y})=\alpha(\boldsymbol{y}), v(\boldsymbol{y})=$ $\mathscr{G}_{\kappa}(\boldsymbol{x}-\boldsymbol{y})$, and $\mathbf{a}=\boldsymbol{n}(\boldsymbol{x}) \times \mathbf{q}(\boldsymbol{x})$, where $\mathbf{q} \in \mathbf{H}^{-1 / 2}(\operatorname{curl}, \Gamma)$ is some tangential vector field depending only on $\boldsymbol{x}$ and hence constant with respect to $\boldsymbol{y}$. This yields

$$
\begin{aligned}
\left\langle\mathbf{q}, \gamma_{\mathrm{R}} \cdot \Upsilon_{\kappa}(\alpha)\right\rangle & =-\int_{\Gamma} \boldsymbol{n}(\boldsymbol{x}) \times \mathbf{q}(\boldsymbol{x}) \cdot\left(\int_{\Gamma}\left(\nabla_{\mathscr{G}_{\kappa}}\right)(\boldsymbol{x}-\boldsymbol{y}) \times \boldsymbol{n}(\boldsymbol{y}) \alpha(\boldsymbol{y}) d \sigma(\boldsymbol{y})\right) d \sigma(\boldsymbol{x}) \\
& =-\int_{\Gamma} \int_{\Gamma} \alpha(\boldsymbol{y})(\boldsymbol{n}(\boldsymbol{x}) \times \mathbf{q}(\boldsymbol{x})) \cdot\left(\boldsymbol{n}(\boldsymbol{y}) \times \nabla_{\boldsymbol{y}} \mathscr{G}_{\kappa}(\boldsymbol{x}-\boldsymbol{y})\right) d \sigma(\boldsymbol{y}) d \sigma(\boldsymbol{x}) \\
& =\int_{\Gamma}(\boldsymbol{n}(\boldsymbol{x}) \times \mathbf{q}(\boldsymbol{x})) \cdot\left(\int_{\Gamma} \mathscr{G}_{\kappa}(\boldsymbol{x}-\boldsymbol{y}) \boldsymbol{n}(\boldsymbol{y}) \times \nabla_{\Gamma} \alpha(\boldsymbol{y}) d \sigma(\boldsymbol{y})\right) d \sigma(\boldsymbol{x})
\end{aligned}
$$

Note that we used $\left(\nabla \mathscr{G}_{\kappa}\right)(\boldsymbol{x}-\boldsymbol{y})=-\nabla_{\boldsymbol{y}}\left(\mathscr{G}_{\kappa}(\boldsymbol{x}-\boldsymbol{y})\right)$ in order to apply (6.15) to the above calculus. Now we point out that $\left(\Delta+\kappa^{2}\right) \Psi_{\kappa}=0$ as $\Psi_{\kappa}$ involves $\mathscr{G}_{\kappa}$. From this we conclude that $\operatorname{curl}^{2} \Psi_{\kappa}(\mathbf{p} \times \boldsymbol{n})=\left(\nabla \operatorname{div}+\kappa^{2}\right) \Psi_{\kappa}(\mathbf{p} \times \boldsymbol{n})$. Taking the trace $\gamma_{\mathrm{R}}$ of this potential and testing with tangential traces yields

$$
\begin{aligned}
\left\langle\mathbf{q}, \gamma_{\mathrm{R}} \cdot \operatorname{curl} \Psi_{\kappa}(\mathbf{p} \times \boldsymbol{n})\right\rangle=-\int_{\Gamma \times \Gamma} \mathscr{G}_{\kappa}(\boldsymbol{x}-\boldsymbol{y})\left[\operatorname{div}_{\Gamma}(\boldsymbol{n}(\boldsymbol{x}) \times \mathbf{q}(\boldsymbol{x})) \operatorname{div}_{\Gamma}(\boldsymbol{n}(\boldsymbol{y}) \times \mathbf{p}(\boldsymbol{y}))\right. \\
\left.-\kappa^{2}(\boldsymbol{n}(\boldsymbol{x}) \times \mathbf{q}(\boldsymbol{x})) \cdot(\boldsymbol{n}(\boldsymbol{y}) \times \mathbf{p}(\boldsymbol{y}))\right] d \sigma(\boldsymbol{x}, \boldsymbol{y}) .
\end{aligned}
$$

Since curl $\Psi_{\kappa}(\mathbf{p} \times \boldsymbol{n})(\boldsymbol{x})=\int_{\Gamma}\left(\nabla \mathscr{G}_{\kappa}\right)(\boldsymbol{x}-\boldsymbol{y}) \times(\mathbf{p}(\boldsymbol{y}) \times \boldsymbol{n}(\boldsymbol{y})) d \sigma(\boldsymbol{y})$, relying once again on Formula $(6.15)$ with $\mathbf{a}=\boldsymbol{n}(\boldsymbol{y}) \times \mathbf{p}(\boldsymbol{y}), u=u(\boldsymbol{x})=\beta(\boldsymbol{x})$ and $v=v(\boldsymbol{x})=\mathscr{G}_{\kappa}(\boldsymbol{x}-\boldsymbol{y})$, we obtain

$$
\left\langle\beta, \gamma_{\mathrm{N}} \cdot \operatorname{curl} \Psi_{\kappa}(\mathbf{p} \times \boldsymbol{n})\right\rangle=\int_{\Gamma \times \Gamma} \mathscr{G}_{\kappa}(\boldsymbol{x}-\boldsymbol{y})(\boldsymbol{n}(\boldsymbol{y}) \times \mathbf{p}(\boldsymbol{y})) \cdot\left(\boldsymbol{n}(\boldsymbol{x}) \times \nabla_{\Gamma} \beta(\boldsymbol{x})\right) d \sigma(\boldsymbol{x}, \boldsymbol{y})
$$

Finally, for arbitrary trace $\mathfrak{v}(\boldsymbol{x})=(\mathbf{q}(\boldsymbol{x}), \beta(\boldsymbol{x})) \in \mathcal{H}_{\mathrm{D}}(\Gamma)$, we obtain the following variational form of $\mathcal{T}_{\mathrm{N}} \cdot \mathcal{D} \mathcal{L}_{\kappa}$ :

$$
\begin{aligned}
\left\langle\left\langle\mathcal{T}_{N} \cdot \mathcal{D} \mathcal{L}_{\kappa}(\mathfrak{u}), \mathfrak{v}\right\rangle\right\rangle= & -\int_{\Gamma \times \Gamma} \mathscr{G}_{\kappa}(\boldsymbol{x}-\boldsymbol{y})\left[\operatorname{div}_{\Gamma}(\boldsymbol{n}(\boldsymbol{x}) \times \mathbf{q}(\boldsymbol{x})) \operatorname{div}_{\Gamma}(\boldsymbol{n}(\boldsymbol{y}) \times \mathbf{p}(\boldsymbol{y}))\right. \\
& \left.-\kappa^{2}(\boldsymbol{n}(\boldsymbol{x}) \times \mathbf{q}(\boldsymbol{x})) \cdot(\boldsymbol{n}(\boldsymbol{y}) \times \mathbf{p}(\boldsymbol{y}))\right] d \sigma(\boldsymbol{x}, \boldsymbol{y}) \\
& +\int_{\Gamma \times \Gamma} \mathscr{G}_{\kappa}(\boldsymbol{x}-\boldsymbol{y})\left[(\boldsymbol{n}(\boldsymbol{x}) \times \mathbf{q}(\boldsymbol{x})) \cdot\left(\boldsymbol{n}(\boldsymbol{y}) \times \nabla_{\Gamma} \alpha(\boldsymbol{y})\right)\right. \\
& \left.+(\boldsymbol{n}(\boldsymbol{y}) \times \mathbf{p}(\boldsymbol{y})) \cdot\left(\boldsymbol{n}(\boldsymbol{x}) \times \nabla_{\Gamma} \beta(\boldsymbol{x})\right)\right] d \sigma(\boldsymbol{x}, \boldsymbol{y}) \\
& +\int_{\Gamma \times \Gamma} \boldsymbol{n}(\boldsymbol{x}) \cdot \mathbb{G}_{\kappa}(\boldsymbol{x}-\boldsymbol{y}) \cdot \boldsymbol{n}(\boldsymbol{y}) \alpha(\boldsymbol{y}) \beta(\boldsymbol{x}) d \sigma(\boldsymbol{x}, \boldsymbol{y}) .
\end{aligned}
$$

The next result describes which parts of the above expression are compact and hence can be "ignored" when investigating coercivity. 
Lemma 6.10. Define $\mathrm{A}_{\mathrm{DL}}: \mathcal{H}_{\mathrm{D}}(\Gamma) \rightarrow \mathcal{H}_{\mathrm{N}}(\Gamma)$ as the continuous operator inducing the following sesqui-linear form:

$$
\begin{aligned}
\left\langle\mathrm{A}_{\mathrm{DL}}(\mathfrak{u}), \mathfrak{v}\right\rangle:= & \mathrm{B}((\boldsymbol{n} \times \mathbf{p}, \alpha),(\boldsymbol{n} \times \mathbf{q}, \beta)), \\
\left.\mathrm{B}\left(\mathbf{p}^{\times}, \alpha\right),\left(\mathbf{q}^{\times}, \beta\right)\right\rangle:=- & \left(\operatorname{div}_{\Gamma} \mathbf{p}^{\times}, \operatorname{div}_{\Gamma} \mathbf{q}^{\times}\right)_{-1 / 2}+\kappa^{2}\left(\mathbf{p}^{\times}, \mathbf{q}^{\times}\right)_{-1 / 2}+ \\
& \left(\operatorname{curl}_{\Gamma} Q_{\star} \alpha, \mathbf{q}^{\times}\right)_{-1 / 2}+\left(\mathbf{p}^{\times}, \operatorname{curl}_{\Gamma} Q_{\star} \beta\right)_{-1 / 2},
\end{aligned}
$$

for $\mathfrak{u}=(\mathbf{p}, \alpha), \mathfrak{v}=(\mathbf{q}, \beta) \in \mathcal{H}_{\mathrm{D}}(\Gamma)$ and $\mathbf{p}^{\times}, \mathbf{q}^{\times} \in \mathbf{H}^{-\frac{1}{2}}(\operatorname{div}, \Gamma), \alpha, \beta \in \mathrm{H}^{1 / 2}(\Gamma)$. Then for any $\kappa \in \mathbb{C}_{+}$the operator $\mathcal{T}_{\mathrm{N}} \cdot \mathcal{D} \mathcal{L}_{\kappa}-\mathrm{A}_{\mathrm{DL}}: \mathcal{H}_{\mathrm{D}}(\Gamma) \rightarrow \mathcal{H}_{\mathrm{N}}(\Gamma)$ is compact and depends continuously on $\kappa$.

Proof:

Note that $\alpha \boldsymbol{n}, \beta \boldsymbol{n} \in \mathrm{L}^{2}(\Gamma)^{3}$ whenever $\alpha, \beta \in \mathrm{H}^{1 / 2}(\Gamma)$. In addition $\mathrm{L}^{2}(\Gamma)^{3}$ is compactly embedded into $\mathbf{H}_{\mathrm{T}}^{-1 / 2}(\Gamma)$, and the bilinear form

$$
\mathbf{p}, \mathbf{q} \mapsto \int_{\Gamma \times \Gamma} \mathbf{p}(\boldsymbol{y}) \cdot \mathbb{G}_{\kappa}(\boldsymbol{x}-\boldsymbol{y}) \cdot \mathbf{q}(\boldsymbol{x}) d \sigma(\boldsymbol{x}, \boldsymbol{y})
$$

is a continuous bilinear form over $\mathbf{H}_{\mathrm{T}}^{-1 / 2}(\Gamma) \times \mathbf{H}_{\mathrm{T}}^{-1 / 2}(\Gamma)$. As a consesquence the third term in Expression (6.16) is compact. Next recall that replacing $\mathscr{G}_{\kappa}$ by $\mathscr{G}_{0}$ in the first two terms of (6.16) only induces a compact perturbation, see e.g. Lemma 3.9 .8 in [32]. After applying this substitution, $A_{D L}$ is obtained by simply re-arranging the first two terms of (6.16) and taking into account $\operatorname{curl}_{\Gamma}=\nabla_{\Gamma} \times \boldsymbol{n}$.

To establish coercivity of the bilinear form (6.16), it suffices to prove a generalized Garding inequality for the sesqui-linear form $\mathrm{B}(\cdot, \cdot)$ defined in (6.17). Let us introduce a bounded linear transformation

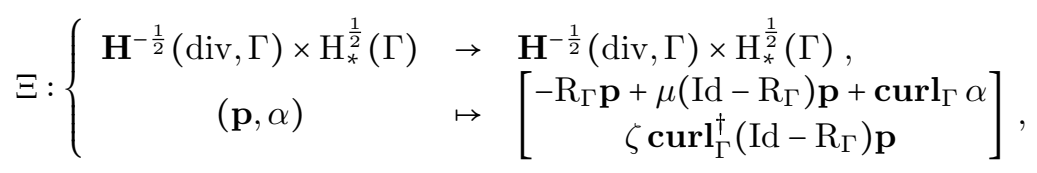

with $\mu \in \mathbb{C},|\mu|=1$, such that $\mathfrak{R}\left\{\bar{\mu} \kappa^{2}\right\}=|\kappa|^{2}$ and $\zeta:=2+|\kappa|^{4}>0$. The operator $\mathrm{R}_{\Gamma}$ was introduced in Lemma 6.5. The operator is bijective with inverse ( $\mathrm{P}_{0}$ defined in (6.6))

$$
\Xi^{-1}(\mathbf{q}, \beta)=\left[\begin{array}{c}
-\mathrm{R}_{\Gamma} \mathbf{q}+\zeta^{-1} \operatorname{curl}_{\Gamma} \beta+\mu^{-1}\left(\operatorname{Id}-\mathrm{P}_{0}\right)\left(\mathrm{Id}-\mathrm{R}_{\Gamma}\right) \mathbf{q} \\
\operatorname{curl}_{\Gamma}^{\dagger}\left(\left(\mathrm{Id}-\mathrm{R}_{\Gamma}\right) \mathbf{q}\right)-\mu \zeta^{-1} \beta
\end{array}\right], \quad \begin{aligned}
& \mathbf{q} \in \mathbf{H}^{-\frac{1}{2}}(\operatorname{div}, \Gamma) \\
& \beta \in \mathrm{H}^{1 / 2}(\Gamma)
\end{aligned}
$$

We call $\Xi_{\times}$a derived isomorphism $\mathbf{H}^{-\frac{1}{2}}(\operatorname{curl}, \Gamma) \times \mathrm{H}_{*}^{\frac{1}{2}}(\Gamma) \rightarrow \mathbf{H}^{-\frac{1}{2}}(\operatorname{curl}, \Gamma) \times \mathrm{H}_{*}^{\frac{1}{2}}(\Gamma)$ that involves rotating the tangential vector arguments before and after the application of $\Xi$.

TheOREM 6.11. For any $\kappa \in \mathbb{C}_{+}$there exists a compact operator $\mathrm{K}_{\kappa}: \mathcal{H}_{\mathrm{D}}(\Gamma) \rightarrow$ $\mathcal{H}_{\mathrm{N}}(\Gamma)$, and a constant $C=C(\kappa)>0$ such that

$$
\left.\mathfrak{R} e\left\{\left\langle\left(\mathcal{T}_{\mathrm{N}} \cdot \mathcal{D} \mathcal{L}_{\kappa}+\mathrm{K}\right) \mathfrak{u}, \Xi(\overline{\mathfrak{u}})\right\rangle\right\}\right\} \geq C\|\mathfrak{u}\|_{\mathcal{H}_{\mathrm{D}}(\Gamma)}^{2} \quad \forall \mathfrak{u} \in \mathcal{H}_{\mathrm{D}}(\Gamma) .
$$

Proof:

Since, according to Lemma 6.10 , the weak form of $\mathcal{T}_{\mathrm{N}} \cdot \mathcal{D} \mathcal{L}_{\kappa}$ agrees with $\mathrm{B}(\cdot, \cdot)$ up to compact perturbations (and isometric rotations), all we have to show is the 
existence of a compact sesqui-linear form $\mathrm{k}_{\kappa}$ on $\mathbf{H}^{-\frac{1}{2}}(\operatorname{div}, \Gamma) \times \mathrm{H}^{1 / 2}(\Gamma)$ and of a constant $C=C(\kappa)>0$ such that

$$
\mathrm{B}((\mathbf{p}, \alpha), \Xi(\mathbf{p}, \alpha))-\mathrm{k}_{\kappa}((\mathbf{p}, \alpha), \Xi(\mathbf{p}, \alpha)) \geq C\left(\|\mathbf{p}\|_{\mathbf{H}^{-\frac{1}{2}}(\operatorname{div}, \Gamma)}^{2}+\|\alpha\|_{\mathrm{H}^{1 / 2}(\Gamma)}^{2}\right)
$$

for all $(\mathbf{p}, \alpha) \in \mathbf{H}^{-\frac{1}{2}}(\operatorname{div}, \Gamma) \times \mathrm{H}^{1 / 2}(\Gamma)$. We find

$$
\begin{aligned}
\mathrm{B}((\mathbf{p}, \alpha), \Xi(\mathbf{p}, \alpha))= & \left\|\operatorname{div}_{\Gamma} \mathrm{R}_{\Gamma} \mathbf{p}\right\|_{-1 / 2}^{2}-\kappa^{2}\left(\mathbf{p}, \mathrm{R}_{\Gamma} \mathbf{p}\right)_{-1 / 2}+\kappa^{2}\left(\mathrm{R}_{\Gamma} \mathbf{p}, \mu\left(\operatorname{Id}-\mathrm{R}_{\Gamma}\right) \mathbf{p}+\operatorname{curl}_{\Gamma} \alpha\right)_{-1 / 2}+ \\
& \kappa^{2} \bar{\mu}\left\|\left(\operatorname{Id}-\mathrm{R}_{\Gamma}\right) \mathbf{p}\right\|_{-1 / 2}^{2}+\kappa^{2}\left(\left(\operatorname{Id}-\mathrm{R}_{\Gamma}\right) \mathbf{p}, \operatorname{curl}_{\Gamma} \alpha\right)_{-1 / 2}- \\
& \left(\operatorname{curl}_{\Gamma} \alpha, \mathrm{R}_{\Gamma} \mathbf{p}\right)_{-1 / 2}+\bar{\mu}\left(\operatorname{curl}_{\Gamma} \alpha,\left(\operatorname{Id}-\mathrm{R}_{\Gamma}\right) \mathbf{p}\right)_{-1 / 2}+ \\
& \left\|\operatorname{curl}_{\Gamma} \alpha\right\|_{-1 / 2}^{2}+\zeta\left(\mathbf{p},\left(\operatorname{Id}-\mathrm{R}_{\Gamma}\right) \mathbf{p}\right)_{-1 / 2} \\
=\left\|\operatorname{div}_{\Gamma} \mathrm{R}_{\Gamma} \mathbf{p}\right\|_{-1 / 2}^{2}+\left(\zeta+\kappa^{2} \bar{\mu}\right)\left\|\left(\operatorname{Id}-\mathrm{R}_{\Gamma}\right) \mathbf{p}\right\|_{-1 / 2}^{2}+\left\|\operatorname{curl}_{\Gamma} \alpha\right\|_{-1 / 2}^{2}+ & \kappa^{2}\left(\left(\operatorname{Id}-\mathrm{R}_{\Gamma}\right) \mathbf{p}, \operatorname{curl}_{\Gamma} \alpha\right)_{-1 / 2}+\bar{\mu}\left(\operatorname{curl}_{\Gamma} \alpha,\left(\operatorname{Id}-\mathrm{R}_{\Gamma}\right) \mathbf{p}\right)_{-1 / 2}+\mathrm{k}_{\kappa}((\mathbf{p}, \alpha), \Xi(\mathbf{p}, \alpha)),
\end{aligned}
$$

with a sesqui-linear form

$$
\begin{aligned}
\mathrm{k}_{\kappa}((\mathbf{p}, \alpha), \Xi(\mathbf{q}, \beta)):= & -\kappa^{2}\left(\mathbf{p}, \mathrm{R}_{\Gamma} \mathbf{q}\right)_{-1 / 2}+\kappa^{2}\left(\mathrm{R}_{\Gamma} \mathbf{p}, \mu\left(\operatorname{Id}-\mathrm{R}_{\Gamma}\right) \mathbf{q}+\operatorname{curl}_{\Gamma} \beta\right)_{-1 / 2}- \\
& \left(\operatorname{curl}_{\Gamma} \alpha, \mathrm{R}_{\Gamma} \mathbf{q}\right)_{-1 / 2}+\zeta\left(\mathrm{R}_{\Gamma} \mathbf{p},\left(\operatorname{Id}-\mathrm{R}_{\Gamma}\right) \mathbf{q}\right)_{-1 / 2}
\end{aligned}
$$

which will inherit compactness from $\mathrm{R}_{\Gamma}$, recall Lemma 6.5 (i). By means of Young's inequality we estimate for arbitrary $\epsilon, \delta>0$

$$
\begin{aligned}
\mathrm{B}((\mathbf{p}, \alpha), & \Xi(\mathbf{p}, \alpha))-\mathrm{k}_{\kappa}((\mathbf{p}, \alpha), \Xi(\mathbf{p}, \alpha)) \\
= & \left\|\operatorname{div}_{\Gamma} \mathrm{R}_{\Gamma} \mathbf{p}\right\|_{-1 / 2}^{2}+\left(\zeta+\kappa^{2} \bar{\mu}\right)\left\|\left(\mathrm{Id}-\mathrm{R}_{\Gamma}\right) \mathbf{p}\right\|_{-1 / 2}^{2}+\left\|\operatorname{curl}_{\Gamma} \alpha\right\|_{-1 / 2}^{2} \\
& \kappa^{2}\left(\left(\operatorname{Id}-\mathrm{R}_{\Gamma}\right) \mathbf{p}, \operatorname{curl}_{\Gamma} \alpha\right)_{-1 / 2}+\bar{\mu}\left(\operatorname{curl}_{\Gamma} \alpha,\left(\mathrm{Id}-\mathrm{R}_{\Gamma}\right) \mathbf{p}\right)_{-1 / 2} \\
\geq & \left\|\operatorname{div}_{\Gamma} \mathrm{R}_{\Gamma} \mathbf{p}\right\|_{-1 / 2}^{2}+\left(\zeta+|\kappa|^{2}\right)\left\|\left(\operatorname{Id}-\mathrm{R}_{\Gamma}\right) \mathbf{p}\right\|_{-1 / 2}^{2}+\left\|\operatorname{curl}_{\Gamma} \alpha\right\|_{-1 / 2}^{2}+ \\
& \frac{1}{2}|\kappa|^{2}\left(\epsilon\left\|\left(\mathrm{Id}-\mathrm{R}_{\Gamma}\right) \mathbf{p}\right\|_{-1 / 2}^{2}+\frac{1}{\epsilon}\left\|\operatorname{curl}_{\Gamma} \alpha\right\|_{-1 / 2}^{2}\right)- \\
& \frac{1}{2}\left(\delta\left\|\left(\mathrm{Id}-\mathrm{R}_{\Gamma}\right) \mathbf{p}\right\|_{-1 / 2}^{2}+\frac{1}{\delta}\left\|\operatorname{curl}_{\Gamma} \alpha\right\|_{-1 / 2}^{2}\right) \\
& \geq\left\|\operatorname{div}_{\Gamma} \mathrm{R}_{\Gamma} \mathbf{p}\right\|_{-1 / 2}^{2}+\left\|\left(\mathrm{Id}-\mathrm{R}_{\Gamma}\right) \mathbf{p}\right\|_{-1 / 2}^{2}+\frac{1}{4}\left\|\operatorname{curl}_{\Gamma} \alpha\right\|_{-1 / 2}^{2},
\end{aligned}
$$

thanks to the choice $\zeta=2+|\kappa|^{4}-|\kappa|^{2}$, when we set $\epsilon=2|\kappa|^{2}, \delta=2$.

Now that we have established a generalized Garding inequality for $\mathcal{T}_{\mathrm{N}} \cdot \mathcal{D} \mathcal{L}_{\kappa}$, we can conclude its invertibility in the same way as for $\mathcal{T}_{\mathrm{D}} \cdot \mathcal{S} \mathcal{L}_{\kappa}$ with Corollary 6.9 , using Theorem 6.11 instead of Theorem 6.8. We do not reproduce the proof since it runs parallel to that of Corollary 6.9.

Corollary 6.12. For any $\kappa \in \mathbb{C}_{+}$the operator $\mathcal{T}_{\mathrm{N}} \cdot \mathcal{D} \mathcal{L}_{\kappa}: \mathcal{H}_{\mathrm{D}}(\Gamma) \rightarrow \mathcal{H}_{\mathrm{N}}(\Gamma)$ is of Fredholm type of index 0 . Moreover, if $\kappa^{2} \in \mathbb{C}_{+} \backslash\left(\Lambda_{\mathrm{R}} \cup \eta \Lambda_{\mathrm{N}}\right)$, then $\mathcal{T}_{\mathrm{N}} \cdot \mathcal{D} \mathcal{L}_{\kappa}$ is an isomorphism.

6.5. Supplement: Invertibility of second-kind boundary integral operators . The boundary integral operators $\mathcal{T}_{\mathrm{D}} \cdot \mathcal{D} \mathcal{L}_{\kappa}: \mathcal{H}_{\mathrm{D}}(\Gamma) \rightarrow \mathcal{H}_{\mathrm{D}}(\Gamma)$ and $\mathcal{T}_{\mathrm{D}} \cdot \mathcal{D} \mathcal{L}_{\kappa}:$ 
$\mathcal{H}_{\mathrm{D}}(\Gamma) \rightarrow \mathcal{H}_{\mathrm{D}}(\Gamma)$ qualify as second-kind, because they supply a bounded endomorphism of a single trace space each. Their invertibility can be established appealing to the well-posedness of both exterior and interior boundary value problems from Section 3.

Proposition 6.13. Let $\Omega \subset \mathbb{R}^{3}$ be a Lipschitz domain.

(i) If $\Omega$ is bounded, then $\mathcal{T}_{\mathrm{D}} \cdot \mathcal{D} \mathcal{L}_{\kappa}: \mathcal{H}_{\mathrm{D}}(\Gamma) \rightarrow \mathcal{H}_{\mathrm{D}}(\Gamma)$ is an ismorphism if $\kappa^{2} \epsilon$ $\mathbb{C}_{+} \backslash\left(\Lambda_{\mathrm{T}} \cup \eta \Lambda_{\mathrm{D}}\right)$, and $\mathcal{T}_{\mathrm{N}} \cdot \mathcal{S} \mathcal{L}_{\kappa}: \mathcal{H}_{\mathrm{N}}(\Gamma) \rightarrow \mathcal{H}_{\mathrm{N}}(\Gamma)$ is an ismorphism if $\kappa^{2} \epsilon$ $\mathbb{C}_{+} \backslash\left(\Lambda_{\mathrm{R}} \cup \eta \Lambda_{\mathrm{N}}\right)$.

(ii) If $\mathbb{R}^{3} \backslash \bar{\Omega}$ is bounded, then $\mathcal{T}_{\mathrm{D}} \cdot \mathcal{D} \mathcal{L}_{\kappa}: \mathcal{H}_{\mathrm{D}}(\Gamma) \rightarrow \mathcal{H}_{\mathrm{D}}(\Gamma)$ is an ismorphism if $\kappa^{2} \in \mathbb{C}_{+} \backslash\left(\Lambda_{\mathrm{R}} \cup \eta \Lambda_{\mathrm{N}}\right)$, and $\mathcal{T}_{\mathrm{N}} \cdot \mathcal{S} \mathcal{L}_{\kappa}: \mathcal{H}_{\mathrm{N}}(\Gamma) \rightarrow \mathcal{H}_{\mathrm{N}}(\Gamma)$ is an ismorphism if $\kappa^{2} \in\left(\Lambda_{\mathrm{T}} \cup \eta \Lambda_{\mathrm{D}}\right)$.

\section{Proof:}

We only prove invertibility $\mathcal{T}_{\mathrm{D}} \cdot \mathcal{D} \mathcal{L}_{\kappa}$ in the case where $\Omega \subset \mathbb{R}^{3}$ is bounded, since all other cases can be treated in a completely similar manner. Assuming that $\kappa^{2} \epsilon$ $\mathbb{C}_{+} \backslash\left(\Lambda_{\mathrm{T}} \cup \eta \Lambda_{\mathrm{D}}\right)$, we already know that $\operatorname{ker}\left(\mathcal{T}_{\mathrm{D}} \cdot \mathcal{D} \mathcal{L}_{\kappa}\right)=\{0\}$ according to Proposition 6.2.

Take any $\mathfrak{p}=(\mathrm{g}, h) \in \mathcal{H}_{\mathrm{D}}(\Gamma)$ and let us prove that there exists $\mathfrak{u} \in \mathcal{H}_{\mathrm{D}}(\Gamma)$ such that $\mathcal{T}_{\mathrm{D}} \cdot \mathcal{D} \mathcal{L}_{\kappa}(\mathfrak{u})=\mathfrak{p}$. According to Proposition 3.7, there exists a unique $\mathbf{v}_{\text {in }} \in \mathbf{X}(\Omega)$ solution to Problem (3.10) with $\mathbf{g}, h$ as boundary conditions. Let us denote $\mathfrak{p}=$ $\mathcal{T}_{\mathrm{N}}\left(\mathbf{v}_{\text {in }}\right)$. Invoking Corollary 3.9 in $\mathbb{R}^{3} \backslash \bar{\Omega}$, we know that there exists $\mathbf{v}_{\text {out }} \in \mathbf{X}_{\text {loc }}\left(\mathbb{R}^{3} \backslash \bar{\Omega}\right)$ satisfying

$$
\left\{\begin{array}{l}
\operatorname{curl}^{2}\left(\mathbf{v}_{\text {out }}\right)-\eta \nabla\left(\operatorname{div} \mathbf{v}_{\text {out }}\right)-\kappa^{2} \mathbf{v}_{\text {out }}=0 \quad \text { in } \Omega, \\
\lim _{\rho \rightarrow \infty} \int_{\partial \mathrm{B}_{\rho}}\left|\operatorname{curl}\left(\mathbf{v}_{\text {out }}\right) \times \boldsymbol{n}_{\rho}-\imath \kappa \boldsymbol{n}_{\rho} \times\left(\mathbf{v}_{\text {out }} \times \boldsymbol{n}_{\rho}\right)\right|^{2} d \sigma_{\rho}=0 \\
\lim _{\rho \rightarrow \infty} \int_{\partial \mathrm{B}_{\rho}}\left|\operatorname{div}\left(\mathbf{v}_{\text {out }}\right)-\imath \tilde{\kappa} \boldsymbol{n}_{\rho} \cdot \mathbf{v}_{\text {out }}\right|^{2} d \sigma_{\rho}=0, \\
\mathcal{T}_{\mathrm{N}, c}\left(\mathbf{v}_{\text {out }}\right)=\mathfrak{p} \quad \text { on } \Gamma,
\end{array}\right.
$$

Finally, define $\mathbf{v}$ by $\left.\mathbf{v}\right|_{\Omega}:=\mathbf{v}_{\text {in }}$ and $\left.\mathbf{v}\right|_{\mathbb{R}^{3} \backslash \bar{\Omega}}:=\mathbf{v}_{\text {out }}$. By construction we have $\left[\mathcal{T}_{N}(\mathbf{v})\right]=0$. Let us set $\mathfrak{u}:=\left[\mathcal{T}_{\mathrm{D}}(\mathbf{v})\right]$. According to the integral representation formula 4.6, we have $\mathbf{v}_{\text {in }}(\boldsymbol{x})=\mathcal{D} \mathcal{L}_{\kappa}\left(\left[\mathcal{T}_{\mathrm{D}}(\mathbf{v})\right]\right)(\boldsymbol{x})+\mathcal{S} \mathcal{L}_{\kappa}\left(\left[\mathcal{T}_{\mathrm{N}}(\mathbf{v})\right]\right)(\boldsymbol{x})$ for all $\boldsymbol{x} \in \Omega$. Applying the trace operator $\mathcal{T}_{\mathrm{D}}$ to this equality, and taking into account that $\left[\mathcal{T}_{\mathrm{N}}(\mathbf{v})\right]=0$ and $\mathfrak{p}=\mathcal{T}_{\mathrm{N}}\left(\mathbf{v}_{\text {in }}\right)$, we finally obtain $\mathcal{T}_{\mathrm{D}} \cdot \mathcal{D} \mathcal{L}_{\kappa}(\mathfrak{u})=\mathfrak{p}$. As a consequence $\mathcal{T}_{\mathrm{D}} \cdot \mathcal{D} \mathcal{L}_{\kappa}$ is onto.

7. Static case $\kappa=0$ : Boundary integral operators for the Hodge-Laplacian. The first-kind boundary integral equations arising in the static limit case, that is, for $\kappa=0$, deserve particular attention. They are connected with boundary value problems for the Hodge Laplacian $\boldsymbol{\Delta}_{\eta}$. To begin with, note that both potentials, $\mathcal{S} \mathcal{L}_{\kappa}$ from (4.9) and $\mathcal{D} \mathcal{L}_{\kappa}$ from (4.11) remain well defined for $\kappa=0$. The same holds true for the boundary integral operators $\mathcal{T}_{\mathrm{D}} \cdot \mathcal{S} \mathcal{L}_{\kappa}$, see (6.9), and $\mathcal{T}_{\mathrm{N}} \cdot \mathcal{D} \mathcal{L}_{\kappa}$, see (6.16).

Although the boundary integral operators $\mathcal{T}_{\mathrm{D}} \cdot \mathcal{S} \mathcal{L}_{\kappa}$ and $\mathcal{T}_{\mathrm{N}} \cdot \mathcal{D} \mathcal{L}_{\kappa}$ are invertible for $I m \kappa^{2}>0$ according to Corollary 6.9 and 6.12, these operators feature non-trivial kernels for $\kappa=0$. In this section we provide a detailed intrinsic analysis of these kernels based on the bilinear forms (6.9) and (6.16). We also propose constraints that restore uniqueness of solutions of the related first-kind boundary integral equations for $\kappa=0$.

Notice that in the static case $\kappa=0$ it is natural to confine ourselves to real-valued fields. Thus, in this section, we work in real Hilbert spaces throughout.

7.1. Static single layer operator. We first focus on the Dirichlet trace of the Hodge Laplace single layer boundary integral operator $\mathcal{T}_{\mathrm{D}} \cdot \mathcal{S} \mathcal{L}_{\kappa}$ and seek to characterize 
$\operatorname{ker}\left(\mathcal{T}_{\mathrm{D}} \cdot \mathcal{S} \mathcal{L}_{0}\right)$. According to $(6.9)$ for any $\mathfrak{u}=(\mathbf{p}, \alpha) \in \mathcal{H}_{\mathrm{N}}(\Gamma)$ and any $\mathfrak{v}=(\mathbf{q}, \beta) \epsilon$ $\mathcal{H}_{\mathrm{N}}(\Gamma)$, we have

$$
\begin{aligned}
-\left\langle\left\langle\mathcal{T}_{\mathrm{D}} \cdot \mathcal{S} \mathcal{L}_{0}(\mathfrak{u}), \mathfrak{v}\right\rangle=\right. & \int_{\Gamma \times \Gamma} \mathscr{G}_{0}(\boldsymbol{x}-\boldsymbol{y})\left[\mathbf{p}(\boldsymbol{y}) \cdot \mathbf{q}(\boldsymbol{x})+\alpha(\boldsymbol{y}) \operatorname{div}_{\Gamma} \mathbf{q}(\boldsymbol{x})\right] d \sigma(\boldsymbol{x}, \boldsymbol{y}) \\
& +\int_{\Gamma \times \Gamma} \mathscr{G}_{0}(\boldsymbol{x}-\boldsymbol{y}) \beta(\boldsymbol{x}) \operatorname{div}_{\Gamma} \mathbf{p}(\boldsymbol{y}) d \sigma(\boldsymbol{x}, \boldsymbol{y}),
\end{aligned}
$$

which defines a symmetric bilinear form.

Assume that $\mathcal{T}_{\mathrm{D}} \cdot \mathcal{S} \mathcal{L}_{0}(\mathfrak{u})=0$ for some $\mathfrak{u} \in \mathcal{H}_{\mathrm{N}}(\Gamma)$. Choosing $\mathbf{q}(\boldsymbol{x})=0$ and $\beta(\boldsymbol{x})=\operatorname{div}_{\Gamma} \mathbf{p}(\boldsymbol{x})$ in $(7.1)$, we see that $\int_{\Gamma \times \Gamma} \mathscr{G}_{0}(\boldsymbol{x}-\boldsymbol{y}) \beta(\boldsymbol{x}) \beta(\boldsymbol{y}) d \sigma(\boldsymbol{x}, \boldsymbol{y})=\|\beta\|_{-1 / 2}^{2}=0$, which implies $\beta=0$. Now taking $\mathfrak{u}=\mathfrak{v}$, we see that $\left\langle\left\langle\mathcal{T}_{\mathrm{D}} \cdot \mathcal{S} \mathcal{L}_{0}(\mathfrak{u}), \mathfrak{v}\right\rangle=\int_{\Gamma \times \Gamma} \mathscr{G}_{0}(\boldsymbol{x}-\right.$ $\boldsymbol{y}) \mathbf{p}(\boldsymbol{x}) \mathbf{p}(\boldsymbol{y}) d \sigma(\boldsymbol{x}, \boldsymbol{y})=0$ which again implies $\mathbf{p}=0$. Finally, applying a surface Green identity we obtain $\left\langle\left\langle\mathcal{T}_{\mathrm{D}} \cdot \mathcal{S} \mathcal{L}_{0}(\mathfrak{u}), \mathfrak{v}\right\rangle\right\rangle=\int_{\Gamma} \mathbf{q} \nabla_{\Gamma} \psi_{0}(\alpha) d \sigma=0$ for all $\mathbf{q} \in \mathbf{H}^{-1 / 2}(\operatorname{div}, \Gamma)$.

We conclude that $\nabla_{\Gamma} \psi_{0}(\alpha)=0$, which is equivalent to $\psi_{0}(\alpha)$ being constant on each connected component of $\Gamma$. Since $\tau_{\mathrm{D}} \cdot \psi_{0}: \mathrm{H}^{-1 / 2}(\Gamma) \rightarrow \mathrm{H}^{1 / 2}(\Gamma)$ is an isomorphism, we obtain the following result.

Lemma 7.1. For any $\mathfrak{u}=(\mathbf{p}, \alpha) \in \mathcal{H}_{\mathrm{N}}(\Gamma)$, we have $\mathcal{T}_{\mathrm{D}} \cdot \mathcal{S L}_{0}(\mathfrak{u})=0$, if and only if $\nabla_{\Gamma}\left(\tau_{\mathrm{D}} \cdot \psi_{0}(\alpha)\right)=0$ and $\mathbf{p}=0$.

A natural question is how to filter out the elements of this finite dimensional kernel so as to stabilise $\mathcal{T}_{\mathrm{D}} \cdot \mathcal{S} \mathcal{L}_{0}$. We have the following elementary result.

LEMma 7.2. The dimension of $\operatorname{ker}\left(\mathcal{T}_{\mathrm{D}} \cdot \mathcal{S} \mathcal{L}_{0}\right)$ is finite and agrees with the zeroth Betti number $\beta_{0}(\Gamma)$ of $\Gamma$, that is, the number of its connected components. Moreover, every element $\mathfrak{u}=(0, \alpha) \in \operatorname{ker}\left(\mathcal{T}_{\mathrm{D}} \cdot \mathcal{S} \mathcal{L}_{0}\right)$ satisfying $\int_{\Gamma} \beta \alpha d \sigma=0$ for all $\beta \in \mathscr{H}_{0}(\Gamma):=$ $\left\{\varphi \in \mathrm{H}^{1 / 2}(\Gamma), \nabla_{\Gamma} \varphi=0\right\}$ vanishes identically, i.e., $\alpha=0$.

\section{Proof:}

Pick $\mathfrak{u}=(0, \alpha) \in \mathcal{H}_{\mathrm{N}}(\Gamma)$ such that $\mathcal{T}_{\mathrm{D}} \cdot \mathcal{S L}_{0}(\mathfrak{u})=0$, and $\int_{\Gamma} \beta \alpha d \sigma=0$ for all $\beta \epsilon$ $\mathscr{H}_{0}(\Gamma)$. According to the discussion above, $\nabla_{\Gamma} \tau_{\mathrm{D}} \cdot \psi_{0}(\alpha)=0$, so that $\tau_{\mathrm{D}} \cdot \psi_{0}(\alpha) \in \mathscr{H}_{0}(\Gamma)$. As a consequence, taking $\beta=\tau_{\mathrm{D}} \cdot \psi_{0}(\alpha)$, we conclude that $\int_{\Gamma} \alpha \tau_{\mathrm{D}} \cdot \psi_{0}(\alpha) d \sigma=0 \Rightarrow \alpha=0$ due to the coercivity of the scalar single layer potential.

Based on the previous result, the boundary integral equation (7.1) can be regularised imposing vanishing mean value constraint on each connected component of $\Gamma$, by means of Lagrange multipliers. Consider $\mathfrak{f}=(\mathrm{g}, h) \in \mathcal{H}_{\mathrm{D}}(\Gamma)$, and assume we are interested in solving the problem

$$
\left\{\begin{array}{l}
\text { Find } \mathfrak{u} \in \mathcal{H}_{\mathrm{N}}(\Gamma) \text { such that } \\
\left\langle\left\langle\mathcal{T}_{\mathrm{D}} \cdot \mathcal{S} \mathcal{L}_{0}(\mathfrak{u}), \mathfrak{v}\right\rangle=\left\langle\langle\mathfrak{f}, \mathfrak{v}\rangle \quad \forall \mathfrak{v} \in \mathcal{H}_{\mathrm{N}}(\Gamma)\right.\right.
\end{array}\right.
$$

Due to the non-trivial kernel $\mathcal{T}_{\mathrm{D}} \cdot \mathcal{S} \mathcal{L}_{0}$, solutions to this formulation are not unique. Moreover, in accordance with the Fredholm alternative, the right hand side $\mathfrak{f}$ must satisfy compatibility conditions to garantee existence of a solution. Instead of (7.2), one may consider the saddle-point problem

$$
\left\{\begin{array}{l}
\text { Find } \mathfrak{u}=(\mathbf{p}, \alpha) \in \mathcal{H}_{\mathrm{N}}(\Gamma) \text { and } \mu \in \mathscr{H}_{0}(\Gamma) \text { such that } \\
\left\langle\left\langle\mathcal{T}_{\mathrm{D}} \cdot \mathcal{S L}_{0}(\mathfrak{u}), \mathfrak{v}\right\rangle+\int_{\Gamma} \mu \beta d \sigma=\left\langle\langle\mathfrak{f}, \mathfrak{v}\rangle \quad \forall \mathfrak{v}=(\mathbf{q}, \beta) \in \mathcal{H}_{\mathrm{N}}(\Gamma)\right.\right. \\
\int_{\Gamma} \lambda \alpha d \sigma=0 \quad \forall \lambda \in \mathscr{H}_{0}(\Gamma) .
\end{array}\right.
$$


According to the standard theory on saddle-point problems, see e.g. [4, Ch. $1 \& 2]$ or [3, Ch. III§4], since $\mathscr{H}_{0}(\Gamma)$ is finite dimensional, Problem (7.3) admits a unique solution, for any right hand side $\mathfrak{f} \in \mathcal{H}_{\mathrm{D}}(\Gamma)$. If, in addition, the compatibility conditions $\left\langle\{\mathfrak{f}, \mathfrak{v}\rangle=0\right.$ for all $\mathfrak{v} \in \operatorname{ker}\left(\mathcal{T}_{\mathrm{D}} \cdot \mathcal{S} \mathcal{L}_{0}\right)$, then the solution to (7.3) is also solution to (7.2). In a sense, (7.3) is a regularised version of (7.2).

7.2. Static hypersingular boundary integral operator. Next, we elaborate on the kernel of the Hodge Laplace hypersingular boundary integral operator $\mathcal{T}_{\mathrm{N}} \cdot \mathcal{D} \mathcal{L}_{0}$. According to (6.16), for any $\mathfrak{u}=(\mathbf{p}, \alpha), \mathfrak{v}=(\mathbf{q}, \beta) \in \mathcal{H}_{\mathrm{D}}(\Gamma)$ it induces the symmetric bilinear form

$$
\begin{aligned}
\left\langle\left\langle\mathcal{T}_{\mathrm{N}} \cdot \mathcal{D} \mathcal{L}_{0}(\mathfrak{u}), \mathfrak{v}\right\rangle=\right. & -\int_{\Gamma \times \Gamma} \mathscr{G}_{0}(\boldsymbol{x}-\boldsymbol{y}) \operatorname{div}_{\Gamma}\left(\mathbf{q}_{\times}(\boldsymbol{x})\right) \operatorname{div}_{\Gamma}\left(\mathbf{p}_{\times}(\boldsymbol{y})\right) d \sigma(\boldsymbol{x}, \boldsymbol{y}) \\
& -\int_{\Gamma \times \Gamma} \mathscr{G}_{0}(\boldsymbol{x}-\boldsymbol{y})\left[\mathbf{q}_{\times}(\boldsymbol{x}) \cdot \boldsymbol{c u r l}_{\Gamma} \alpha(\boldsymbol{y})+\mathbf{p}_{\times}(\boldsymbol{y}) \cdot \operatorname{curl}_{\Gamma} \beta(\boldsymbol{x})\right] d \sigma(\boldsymbol{x}, \boldsymbol{y}) \\
& +\int_{\Gamma \times \Gamma} \mathscr{G}_{0}(\boldsymbol{x}-\boldsymbol{y}) \alpha(\boldsymbol{y}) \beta(\boldsymbol{x}) \boldsymbol{n}(\boldsymbol{x}) \cdot \boldsymbol{n}(\boldsymbol{y}) d \sigma(\boldsymbol{x}, \boldsymbol{y}) .
\end{aligned}
$$

where, for the sake of brevity, we have denoted $\mathbf{p}_{\times}(\boldsymbol{y}):=\boldsymbol{n}(\boldsymbol{y}) \times \mathbf{p}(\boldsymbol{y})$ and $\mathbf{q}_{\times}(\boldsymbol{x}):=$ $n(x) \times \mathbf{q}(y)$.

Let us examine the elements of the kernel of this operator. Assume that $\mathcal{T}_{\mathrm{N}}$. $\mathcal{D} \mathcal{L}_{0}(\mathfrak{u})=0$.

(i) Taking $\beta=0$ and $\mathbf{q}=\nabla_{\Gamma} \alpha$, so that $\mathbf{q}_{\times}=-\operatorname{curl}_{\Gamma} \alpha$, we obtain

$$
0=\int_{\Gamma \times \Gamma} \mathscr{G}_{0}(\boldsymbol{x}-\boldsymbol{y}) \mathbf{q}_{\times}(\boldsymbol{x}) \cdot \mathbf{q}_{\times}(\boldsymbol{y}) d \sigma(\boldsymbol{x}, \boldsymbol{y}) \quad \Rightarrow \quad \mathbf{q}_{\times}=\boldsymbol{n} \times \nabla_{\Gamma} \alpha=0,
$$

thanks to the coercivity of the vector single layer potential, see [9, Lemma 8].

(ii) Now taking $\mathbf{q}=0$ and $\beta=\alpha$, we obtain $\int_{\Gamma \times \Gamma} \mathscr{G}_{0}(\boldsymbol{x}-\boldsymbol{y}) \alpha(\boldsymbol{y}) \alpha(\boldsymbol{x}) \boldsymbol{n}(\boldsymbol{x})$. $\boldsymbol{n}(\boldsymbol{y}) d \sigma(\boldsymbol{x}, \boldsymbol{y})=0$ and so $\alpha=0$.

(iii) Taking $\mathbf{q}=\mathbf{p}$ and $\beta=0$, then leads to $\int_{\Gamma \times \Gamma} \mathscr{G}_{0}(\boldsymbol{x}-\boldsymbol{y}) \operatorname{curl}_{\Gamma}(\mathbf{p}(\boldsymbol{x})) \cdot \operatorname{curl}_{\Gamma}(\mathbf{p}(\boldsymbol{y})) d \sigma(\boldsymbol{x}, \boldsymbol{y})=$ 0 , which implies $\operatorname{curl}_{\Gamma}(\mathbf{p})=0$.

(iv) Finally, applying a surface Green formula yields

$0=\int_{\Gamma} \operatorname{curl}_{\Gamma} \beta(\boldsymbol{x}) \cdot\left(\int_{\Gamma} \mathscr{G}_{0}(\boldsymbol{x}-\boldsymbol{y}) \boldsymbol{n}(\boldsymbol{y}) \times \mathbf{p}(\boldsymbol{y}) d \sigma(\boldsymbol{y})\right) d \sigma(\boldsymbol{x})=\int_{\Gamma} \beta \operatorname{div}_{\Gamma}\left(\Psi_{0, \times}(\mathbf{p})\right) d \sigma$ for all $\beta \in \mathrm{H}^{1 / 2}(\Gamma)$, where

$$
\Psi_{0, \times}(\mathbf{p})(\boldsymbol{x}):=-\boldsymbol{n}(\boldsymbol{x}) \times \int_{\Gamma} \mathscr{G}_{0}(\boldsymbol{x}-\boldsymbol{y}) \boldsymbol{n}(\boldsymbol{y}) \times \mathbf{p}(\boldsymbol{y}) d \sigma(\boldsymbol{y}) .
$$

From this we conclude that we must have $\operatorname{div}_{\Gamma} \Psi_{0, \times}(\mathbf{p})=0$. To summarize we have proved the following result.

Lemma 7.3. For any $\mathfrak{u}=(\mathbf{p}, \alpha) \in \mathcal{H}_{\mathrm{D}}(\Gamma)$, we have $\mathcal{T}_{\mathrm{N}} \cdot \mathcal{D} \mathcal{L}_{0}(\mathfrak{u})=0$ if and only if $\alpha=0, \operatorname{div}_{\Gamma} \Psi_{0, \times}(\mathbf{p})=0$ and $\operatorname{curl}_{\Gamma}(\mathbf{p})=0$.

Although this characterization of the kernel of $\mathcal{T}_{\mathrm{N}} \cdot \mathcal{D} \mathcal{L}_{0}$ does not seem very convenient, the next result shows that it is finite dimensional, at least.

LEMma 7.4. The dimension of $\operatorname{ker}\left(\mathcal{T}_{\mathrm{N}} \cdot \mathcal{D} \mathcal{L}_{0}\right)$ is finite and agrees with the first Betti number $\beta_{1}(\Gamma)$ of $\Gamma$, that is, the number of equivalence classes of non-bounding cycles in $\Gamma$. Moreover every element $\mathfrak{u}=(\mathbf{p}, 0) \in \operatorname{ker}\left(\mathcal{T}_{\mathrm{N}} \cdot \mathcal{D} \mathcal{L}_{0}\right)$ satisfying

$\int_{\Gamma} \mathbf{p} \cdot \mathbf{q} d \sigma=0 \quad \forall \mathbf{q} \in \mathscr{H}_{1}(\Gamma):=\left\{\mathbf{w} \in \mathbf{H}^{-1 / 2}\left(\operatorname{curl}_{\Gamma}, \Gamma\right) \cap \mathbf{H}^{-1 / 2}\left(\operatorname{div}_{\Gamma}, \Gamma\right), \operatorname{div}_{\Gamma} \mathbf{w}=\operatorname{curl}_{\Gamma} \mathbf{w}=0\right\}$

vanishes, i.e., $\mathbf{p}=0$. 


\section{Proof:}

For the sake of conciseness, denote $\mathbf{H}^{-1 / 2}\left(\operatorname{curl}_{\Gamma} 0\right):=\left\{\mathbf{v} \in \mathbf{H}^{-1 / 2}(\operatorname{curl}, \Gamma), \operatorname{curl}_{\Gamma} \mathbf{v}=\right.$ $0\}$, and $\mathscr{H}_{\times}(\Gamma):=\left\{\mathbf{v} \in \mathbf{H}^{-1 / 2}\left(\operatorname{curl}_{\Gamma} 0\right), \operatorname{div}_{\Gamma} \Psi_{0, \times}(\mathbf{v})=0\right\}$. We have to prove that the bilinear form $\mathbf{u}, \mathbf{v} \mapsto \int_{\Gamma} \mathbf{u} \cdot \mathbf{v} d \sigma$ is non degenerate over $\mathscr{H}_{x}(\Gamma) \times \mathscr{H}_{1}(\Gamma)$.

Observe that both $\mathscr{H}_{\times}(\Gamma)$ and $\mathscr{H}_{1}(\Gamma)$ are closed subspaces of $\mathbf{H}^{-1 / 2}\left(\operatorname{curl}_{\Gamma} 0\right)$. Besides $\mathscr{H}_{1}(\Gamma) \subset \mathrm{L}_{\mathrm{T}}^{2}(\Gamma)$ where $\mathrm{L}_{\mathrm{T}}^{2}(\Gamma)$ refers to the space of square integrable tangential vector fields over $\Gamma$. This inclusion stems from the Hodge Decomposition of square integrable differential forms on $\Gamma$ [33, Sect.2.4] which implies $C\|\mathbf{u}\|_{\mathrm{L}^{2}(\Gamma)} \leq$ $\left\|\operatorname{div}_{\Gamma} \mathbf{u}\right\|_{\mathrm{H}^{-1}(\Gamma)}+\left\|\operatorname{curl}_{\Gamma} \mathbf{u}\right\|_{\mathbf{H}^{-1}(\Gamma)}+\|\mathbf{u}\|_{\mathrm{H}^{-1}(\Gamma)} \forall \mathbf{u} \in \mathrm{L}_{\mathrm{T}}^{2}(\Gamma)$ for some positive constant $C>$ 0 . Also observe that $\mathbf{u}, \mathbf{v} \mapsto \int_{\Gamma} \mathbf{u} \cdot \Psi_{0, \times}(\overline{\mathbf{v}}) d \sigma$ yields a scalar product on $\mathbf{H}^{-1 / 2}\left(\operatorname{curl}_{\Gamma} 0\right)$. The direct sum

$$
\mathbf{H}^{-1 / 2}\left(\operatorname{curl}_{\Gamma} 0\right)=\mathscr{H}_{\times}(\Gamma) \oplus \nabla_{\Gamma} \mathrm{H}^{1 / 2}(\Gamma)
$$

is orthogonal with respect to this scalar product, by the very definition of $\mathscr{H}_{x}(\Gamma)$. Next consider the projection $\mathcal{S}: \mathscr{H}_{1}(\Gamma) \rightarrow \mathscr{H}_{x}(\Gamma)$ that is orthogonal with respect to this scalar product. For any $\mathbf{u} \in \mathscr{H}_{1}(\Gamma)$, the field $\mathcal{S}(\mathbf{u})$ is the only solution to

$$
\left\{\begin{array}{l}
\mathcal{S}(\mathbf{u}) \in \mathscr{H}_{\times}(\Gamma) \quad \text { such that } \\
\int_{\Gamma} \mathcal{S}(\mathbf{u}) \cdot \Psi_{0, \times}(\overline{\mathbf{v}}) d \sigma=\int_{\Gamma} \mathbf{u} \cdot \Psi_{0, \times}(\overline{\mathbf{v}}) d \sigma \quad \forall \mathbf{v} \in \mathscr{H}_{\times}(\Gamma) .
\end{array}\right.
$$

Let us show that $\mathcal{S}$ is an isomorphism. First of all, if $\mathbf{u} \in \mathscr{H}_{1}(\Gamma)$ satisfies $\mathcal{S}(\mathbf{u})=0$, then according to (7.6) and (7.7), we have $\mathbf{u}=\nabla_{\Gamma} \varphi$ for some $\varphi \in \mathrm{H}^{1 / 2}(\Gamma)$. Since $\mathscr{H}_{1}(\Gamma) \subset \mathrm{L}_{\mathrm{T}}^{2}(\Gamma)$ and $\operatorname{div}_{\Gamma} \mathbf{u}=0$, we conclude that $\|\mathbf{u}\|_{\mathrm{L}^{2}(\Gamma)}^{2}=\int_{\Gamma} \bar{\varphi} \operatorname{div}_{\Gamma}(\mathbf{u}) d \boldsymbol{x}=0$.

Next we prove that $\mathcal{S}$ is onto. Pick any $\mathbf{u} \in \mathscr{H}_{\times}(\Gamma)$. As a consequence of the decomposition $\mathbf{H}(\operatorname{curl}, \Omega)=\mathrm{H}^{1}(\Omega)^{3}+\nabla \mathrm{H}^{1}(\Omega)$, see e.g. [18, Lemma 2.4], we know that $\mathbf{H}^{-1 / 2}(\operatorname{curl}, \Gamma)=\mathbf{H}_{\mathrm{T}}^{1 / 2}(\Gamma)+\nabla_{\Gamma} \mathrm{H}^{1 / 2}(\Gamma)$, where $\mathbf{H}_{\mathrm{T}}^{1 / 2}(\Gamma)$ was introduced in $\S 2.4$, so there exist $\mathbf{u}^{\prime} \in \mathrm{L}_{\mathrm{T}}^{2}(\Gamma)$ and $p \in \mathrm{H}^{1 / 2}(\Gamma)$ such that $\mathbf{u}=\mathbf{u}^{\prime}-\nabla_{\Gamma} p$. Obviously $\operatorname{curl}_{\Gamma}\left(\mathbf{u}^{\prime}\right)=$ $\operatorname{curl}_{\Gamma}(\mathbf{u})=0$. On the other hand, if $p^{\prime}$ refers to the unique element of $\mathrm{H}^{1}(\Gamma)$ satisfying $\int_{\Gamma} p^{\prime} d \sigma=0$ and $\int_{\Gamma} \nabla_{\Gamma} p^{\prime} \cdot \nabla_{\Gamma} q d \sigma=\int_{\Gamma} \mathbf{u}^{\prime} \cdot \nabla_{\Gamma} q d \sigma \forall q \in \mathrm{H}^{1}(\Gamma)$, then $\mathbf{u}^{\prime \prime}:=\mathbf{u}^{\prime}+\nabla_{\Gamma} p^{\prime}=$ $\mathbf{u}+\nabla_{\Gamma}\left(p+p^{\prime}\right) \in \mathscr{H}_{1}(\Gamma)$. As a consequence we have $\int_{\Gamma}\left(\mathbf{u}^{\prime \prime}-\mathbf{u}\right) \cdot \Psi_{0, \times}(\overline{\mathbf{v}}) d \sigma=0$ for all $\mathbf{v} \in \mathscr{H}_{\times}(\Gamma)$, which means $\mathcal{S}\left(\mathbf{u}^{\prime \prime}\right)=\mathbf{u}$.

We have just proved that $\mathcal{S}$ is an isomorphism. Since $\mathscr{H}_{1}(\Gamma)$ is finite dimensional, we have $\operatorname{dim}\left(\operatorname{ker}\left(\mathcal{T}_{\mathrm{N}} \cdot \mathcal{D} \mathcal{L}_{0}\right)\right)=\operatorname{dim} \mathscr{H}_{\times}(\Gamma)=\operatorname{dim} \mathscr{H}_{1}(\Gamma)=\beta_{1}(\Gamma)$. Finally, consider any $\mathbf{u} \in \mathscr{H}_{\times}(\Gamma)$ satisfying $\int_{\Gamma} \mathbf{u} \cdot \mathbf{v} d \sigma=0$ forall $\mathbf{v} \in \mathscr{H}_{1}(\Gamma)$. There exists a unique $\mathbf{u}^{\prime} \in \mathscr{H}_{1}(\Gamma)$ such that $\mathbf{u}=\mathcal{S}\left(\mathbf{u}^{\prime}\right)$ and, according to (7.6)-(7.7) we have $\mathbf{u}-\mathbf{u}^{\prime} \in \nabla_{\Gamma} \mathrm{H}^{1 / 2}(\Gamma)$. In particular, we have $0=\int_{\Gamma} \mathbf{u} \cdot \mathbf{u}^{\prime} d \sigma=\int_{\Gamma}\left|\mathbf{u}^{\prime}\right|^{2} d \sigma$, so that $\mathbf{u}^{\prime}=0$ and $\mathbf{u} \in \mathscr{H}_{\times}(\Gamma) \cap$ $\nabla_{\Gamma} \mathrm{H}^{1 / 2}(\Gamma)=\{0\}$.

Now let us consider a right hand side $\mathfrak{f}=(\mathrm{g}, h) \in \mathcal{H}_{\mathrm{N}}(\Gamma)$, and assume we are interested in solving the problem

$$
\left\{\begin{array}{l}
\text { Find } \mathfrak{u} \in \mathcal{H}_{\mathrm{D}}(\Gamma) \text { such that } \\
\left\langle\mathcal{T}_{\mathrm{N}} \cdot \mathcal{D} \mathcal{L}_{0}(\mathfrak{u}), \mathfrak{v}\right\rangle=\langle\mathfrak{f}, \mathfrak{v}\rangle \quad \forall \mathfrak{v} \in \mathcal{H}_{\mathrm{D}}(\Gamma)
\end{array}\right.
$$

Here again the above problem is of Fredholm type with index 0 , but the corresponding operator admits a non-trivial kernel. This kernel can be suppressed by imposing appropriate constraints by means of Lagrange multipliers. We are led to the saddle- 
point problem

$$
\left\{\begin{array}{l}
\text { Find } \mathfrak{u}=(\mathbf{p}, \alpha) \in \mathcal{H}_{\mathrm{D}}(\Gamma) \text { and } \boldsymbol{\mu} \in \mathscr{H}_{1}(\Gamma) \text { such that } \\
\left\langle\mathcal{T}_{\mathrm{N}} \cdot \mathcal{D} \mathcal{L}_{0}(\mathfrak{u}), \mathfrak{v}\right\rangle+\int_{\Gamma} \boldsymbol{\mu} \cdot \mathbf{q} d \sigma=\langle\mathfrak{f}, \mathfrak{v}\rangle \quad \forall \mathfrak{v}=(\mathbf{q}, \beta) \in \mathcal{H}_{\mathrm{D}}(\Gamma) \\
\int_{\Gamma} \boldsymbol{\lambda} \cdot \mathbf{p} d \sigma=0 \quad \forall \lambda \in \mathscr{H}_{1}(\Gamma)
\end{array}\right.
$$

Once again standard theory of saddle-point problems, see [4, Ch. 1\&2] or [3, Chap. III§4], shows that the problem above admits a unique solution that is also solution to (7.8) in the case where $\langle\mathfrak{f}, \mathfrak{v}\rangle=0$ for all $\mathfrak{v} \in \operatorname{ker}\left(\mathcal{T}_{\mathrm{N}} \cdot \mathcal{D} \mathcal{L}_{0}\right)$.

Acknowledgement. X. Claeys acknowledges support through ANR research Grant ANR-15-CE23-0017-01. R. Hiptmair completed work on this manuscript during a stay at LJLL, UPMC, Paris. The authors also would like to thank Dirk Pauly for valuable information about the analysis of the Hodge-Helmholtz operator on unbounded domains.

\section{REFERENCES}

[1] C. Amrouche, C. Bernardi, M. Dauge, and V. Girault. Vector potentials in three-dimensional non-smooth domains. Math. Methods Appl. Sci., 21(9):823-864, 1998.

[2] S. Bauer, D. Pauly, and M. Schomburg. The Maxwell compactness property in bounded weak Lipschitz domains with mixed boundary conditions. SIAM J. Math. Anal., 48(4):29122943, 2016.

[3] D. Braess. Finite elements. Cambridge University Press, Cambridge, third edition, 2007. Theory, Fast Solvers, and Applications in Elasticity Theory.

[4] F. Brezzi and M. Fortin. Mixed and hybrid finite element methods, volume 15 of Springer Series in Computational Mathematics. Springer, New York, 1991.

[5] A. Buffa and P. Ciarlet, Jr. On traces for functional spaces related to Maxwell's equations. I. An integration by parts formula in Lipschitz polyhedra. Math. Methods Appl. Sci., 24(1):9-30, 2001.

[6] A. Buffa and P. Ciarlet, Jr. On traces for functional spaces related to Maxwell's equations. II. Hodge decompositions on the boundary of Lipschitz polyhedra and applications. Math. Methods Appl. Sci., 24(1):31-48, 2001.

[7] A. Buffa, M. Costabel, and C. Schwab. Boundary element methods for Maxwell's equations on non-smooth domains. Numer. Math., 92(4):679-710, 2002.

[8] A. Buffa, M. Costabel, and D. Sheen. On traces for $\mathbf{H}(\mathbf{c u r l}, \Omega)$ in Lipschitz domains. J. Math. Anal. Appl., 276(2):845-867, 2002.

[9] A. Buffa and R. Hiptmair. Galerkin boundary element methods for electromagnetic scattering. In Topics in computational wave propagation, volume 31 of Lect. Notes Comput. Sci. Eng., pages 83-124. Springer, Berlin, 2003.

[10] A. Buffa, R. Hiptmair, T. von Petersdorff, and C. Schwab. Boundary element methods for Maxwell transmission problems in Lipschitz domains. Numer. Math., 95(3):459-485, 2003.

[11] W.C. Chew. Waves and Fields in Inhomogeneous Media. Van Nostrand Reinhold, New York, 1990.

[12] W.C. Chew. Vector potential electromagnetics with generalized gauge for inhomogeneous media: Formulation. Progress in Electromagnetics Research, 149:69-84, 2014.

[13] D. Colton and R. Kress. Inverse Acoustic and Electromagnetic Scattering Theory, volume 93 of Applied Mathematical Sciences. Springer, Heidelberg, 2nd edition, 2013.

[14] M. Costabel. Boundary integral operators on Lipschitz domains: elementary results. SIAM J. Math. Anal., 19(3):613-626, 1988.

[15] M. Costabel and M. Dauge. Singularities of electromagnetic fields in polyhedral domains. Arch. Ration. Mech. Anal., 151(3):221-276, 2000.

[16] H. Federer. Geometric measure theory. Die Grundlehren der mathematischen Wissenschaften, Band 153. Springer, New York, 1969.

[17] C. Hazard and M. Lenoir. On the solution of time-harmonic scattering problems for Maxwell's equations. SIAM J. Math. Anal., 27(6):1597-1630, 1996.

[18] R. Hiptmair. Finite elements in computational electromagnetism. Acta Numer., 11:237-339, 2002. 
[19] G.C. Hsiao and W.L. Wendland. Boundary integral equations, volume 164 of Applied Mathematical Sciences. Springer, Berlin, 2008.

[20] P. Kuhn and D. Pauly. Regularity results for generalized electro-magnetic problems. Analysis (Munich), 30(3):225-252, 2010.

[21] S. Kurz and B. Auchmann. Differential forms and boundary integral equations for maxwell-type problems. In Ulrich Langer, Martin Schanz, Olaf Steinbach, and Wolfgang L. Wendland, editors, Fast Boundary Element Methods in Engineering and Industrial Applications, volume 63 of Lecture Notes in Applied and Computational Mechanics, pages 1-62. Springer Berlin / Heidelberg, 2012.

[22] R.C. MacCamy and E. Stephan. Solution procedures for three-dimensional eddy current problems. J. Math. Anal. Appl., 101(2):348-379, 1984.

[23] W. McLean. Strongly elliptic systems and boundary integral equations. Cambridge University Press, Cambridge, 2000.

[24] D. Mitrea, I. Mitrea, M. Mitrea, and M. Taylor. The Hodge-Laplacian, volume 64 of De Gruyter Studies in Mathematics. DeGruyter, Berlin, 2016.

[25] D. Mitrea, M. Mitrea, and M. Taylor. Layer potentials, the Hodge Laplacian, and global boundary problems in nonsmooth Riemannian manifolds, volume 713 of Memoirs of the American Mathematical Society. American Mathematical Society, Providence, RI, 2001.

[26] P. Monk. Finite element methods for Maxwell's equations. Numerical Mathematics and Scientific Computation. Oxford University Press, New York, 2003.

[27] J.-C. Nédélec and J. Planchard. Une méthode variationnelle d'éléments finis pour la résolution numérique d'un problème extérieur dans $R^{3}$. Rev. Française Automat. Informat. Recherche Opérationnelle Sér. Rouge, 7(R-3):105-129, 1973.

[28] D. Pauly. Low frequency asymptotics for time-harmonic generalized Maxwell's equations in nonsmooth exterior domains. Adv. Math. Sci. Appl., 16(2):591-622, 2006.

[29] R. Picard. An elementary proof for a compact imbedding result in generalized electromagnetic theory. Math. Z., 187(2):151-164, 1984.

[30] R. Picard, N. Weck, and K.-J. Witsch. Time-harmonic Maxwell equations in the exterior of perfectly conducting, irregular obstacles. Analysis (Munich), 21(3):231-263, 2001.

[31] M. Reissel. On a transmission boundary-value problem for the time-harmonic Maxwell equations without displacement currents. SIAM J. Math. Anal., 24(6):1440-1457, 1993.

[32] S.A. Sauter and C. Schwab. Boundary element methods, volume 39 of Springer Series in Computational Mathematics. Springer, Berlin, 2011.

[33] G. Schwarz. Hodge Decomposition - A method for Solving Boundary Value Problems, volume 1607 of Springer Lecture Notes in Mathematics. Springer, Berlin, 1995.

[34] O. Steinbach. Numerical approximation methods for elliptic boundary value problems. Finite and boundary elements. Springer, New York, 2008.

[35] N. Weck. Maxwell's boundary value problem on Riemannian manifolds with nonsmooth boundaries. J. Math. Anal. Appl., 46:410-437, 1974.

[36] N. Weck. Traces of differential forms on Lipschitz boundaries. Analysis. International Mathematical Journal of Analysis and its Applications, 24(2):147-169, 2004.

[37] N. Weck and K.J. Witsch. Generalized linear elasticity in exterior domains. I. Radiation problems. Math. Methods Appl. Sci., 20(17):1469-1500, 1997. 